\title{
Neoclassical, Semi-collisional Tearing Mode Theory in an Axisymmetric Torus
}

\author{
J W Connor ${ }^{1,2} \dagger$, R J Hastie ${ }^{1}$ and P Helander ${ }^{3}$ \\ 1 CCFE, Culham Science Centre, Abingdon, Oxon, UK, OX14 3DB \\ 2 Imperial College of Science and Technology and Medicine, London SW7 2BZ \\ 3 Max-Planck-Institut für Plasmaphysik, 17491 Greifswald, Germany \\ $\dagger$ Email for correspondence:jacku.connor@ukaea.uk
}

\begin{abstract}
A set of layer equations for determining the stability of semi-collisional tearing modes in an axisymmetric torus, incorporating neoclassical physics, in the small ion Larmor radius limit is provided. These can be used as an inner layer module for inclusion in numerical codes that asymptotically match the layer to toroidal calculations of the tearing mode stability index, $\Delta^{\prime}$. They are more complete than in earlier work and comprise equations for the perturbed electron density and temperature, the ion temperature, Ampère's law and the vorticity equation, amounting to a tenth order set of radial differential equations. While the toroidal geometry is kept quite general when treating the classical and Pfirsch-Schlüter transport, parallel bootstrap current and semi-collisional physics, it is assumed that the fraction of trapped particles is small for the banana regime contribution. This is to justify the use of a model collision term when acting on the localised (in velocity space) solutions that remain after the Spitzer solutions have been exploited to account for the bulk of the passing distributions. In this respect, unlike standard neoclassical transport theory, the calculation involves the second Spitzer solution connected with a parallel temperature gradient, because this stability problem involves parallel temperature gradients that cannot occur in equilibrium toroidal transport theory. Furthermore, a calculation of the linearized neoclassical radial transport of toroidal momentum for general geometry is required to complete the vorticity equation. The solutions of the resulting set of equations do not match properly to the ideal MHD equations at large distances from the layer, and a further, intermediate layer involving ion corrections to the electrical conductivity and ion parallel thermal transport is invoked to achieve this matching and allow one to correctly calculate the layer $\Delta^{\prime}$.
\end{abstract}

\section{Introduction}

A number of phenomena in tokamaks, such as the saw-tooth oscillations, plasma disruptions and confinement degradation, appear to involve tearing 
mode activity. Studies of the linear tearing stability for hot plasma have exploited the separation of scales between a narrow 'inner' radial region around a resonant surface where reconnection processes occur and the remaining 'external' region where a marginal ideal MHD model is adequate. The ideal MHD solution, characterised by a quantity $\Delta^{\prime}$, is matched to a corresponding quantity $\hat{\Delta}(\omega+\mathrm{i} \gamma)$ calculated from the inner solution in order to determine the mode frequency $\omega$ and growth rate, $\gamma$. Whereas early studies of linear stability used a simple resistive MHD model for the inner layer, present day, hot, tokamaks require a much more complete physics model.

In a previous paper (Connor et al. 2009) we presented layer equations for determining the stability of semi-collisional tearing modes in a toroidal plasma in the banana regime of collisionality $\left(v_{j, e f f}<\omega_{b j}\right.$ where $v_{j, \text { eff }} \sim v_{j} / f_{t}^{2}$ is the effective collision frequency and $\omega_{b j} \sim f_{t} v_{t h j} / L_{c}$ the bounce frequency of a particle of species $j, \quad f_{t} \ll<1$ being the fraction of trapped particles, $v_{j}$ the frequency for 90-degree Coulomb collisions and $L_{c}$ the connection length around the torus), therefore incorporating neoclassical physics. The semicollisional ordering involves the balance: $\omega \sim k_{\|}^{2} v_{\text {the }}^{2} / v_{e}$, where $k_{\|}=k_{\theta} x / L_{s}$ is the wavenumber parallel to the magnetic field ( $k_{\theta}$ is a poloidal wavenumber, $x$ is the distance from a rational surface and $L_{s}$ is the magnetic shear length), $v_{\text {the }}$ is the electron thermal speed and $v_{e}$ is the electron 90-degree collision frequency, so that parallel transport processes compete with the mode frequency, $\omega$. This balance serves to define the semi-collisional width, $\delta_{e}=\left(\omega v_{e} L_{s}^{2} / k_{\theta}^{2} v_{e}^{2}\right)^{1 / 2}$.

These equations were formulated for general axisymmetric geometry, thus in this respect extending the work of Fitzpatrick (Fitzpatrick 1989). A consistent ordering for semi-collisional theory requires that, as well as the inclusion of parallel collisional transport processes, one should also incorporate collisional cross-field transport. The equations of Fitzpatrick (Fitzpatrick 1989) did indeed include both of these transport processes, albeit using a simplified model collision operator. While Connor et al. (Connor et al. 2009) discussed the role of cross-field transport based on a Lorentz collision operator, thus ignoring likeparticle collisions, the emphasis was on the basic semi-collisional physics and these effects were ignored in the bulk of the paper. The role of the present paper 
is to rectify this limitation by providing a general axisymmetric formulation, including cross-field transport which can be used as a semi-collisional tearing mode layer module for inclusion in numerical codes that asymptotically match the layer to toroidal calculations of the tearing mode stability index, $\Delta^{\prime}$ (Glasser et al. 1975). We also include some additional, relatively small, effects in the electron continuity and thermal equations arising from the poloidal magnetic drift that were ignored by Connor et al. (Connor et al. 2009).

The model pitch-angle scattering collision operator used by Fitzpatrick (Fitzpatrick 1989) provides a good description for distribution functions localised in velocity space around the trapped particle regions, but electric fields, parallel pressure gradients and thermal forces due to parallel temperature gradients generate distortions of the whole passing particle region. Nevertheless, this can be circumvented by the use of the Spitzer functions (Cohen et al. 1950, Spitzer \& Harm 1953) to account for these drives, as demonstrated by Rosenbluth et al. (Rosenbluth et al. 1972), Connor et al. (Connor et al. 1973) and Helander and Sigmar (Helander \& Sigmar 2002); we shall also adopt this approach here. The calculation closely follows neoclassical transport theory but differs in one respect. In equillibrium the electron density and temperature are constant on a flux surface and the only parallel driving force is due to the toroidal electric field, resulting in a role for the Spitzer function related to electrical conductivity. In stability theory, however, parallel gradients of both density and electron temperature can persist, leading to the need to involve the Spitzer function describing the parallel heat flux. The calculation below makes one assumption, namely that the fraction of trapped particles is small. This can be relaxed, albeit leading to more complex algebra, but the distribution functions become less localised and the asymptotic accuracy of the approach is compromised. The derivation of the vorticity equation calls for an expression for the neoclassical radial transport of toroidal momentum and we extend previous work by Wong and Chan (Wong \& Chan 2005) to cover more general geometry, though needing to use a model collision operator in order to determine the required adjoint function.

In Section 2 we introduce the gyro-kinetic model for electrons and ions. An appropriate ordering scheme is used to obtain solutions for the ion and electron distribution functions in Section 3. To complete these solutions, equations for the perturbed densities and temperature of the two species are required. These 
- are obtained in Section 4. The numerical values of various averages of collision frequencies that are required in Sections 3 and 4 are listed in Appendix A. Section 5 develops Ampère's law and the vorticity equation to complete the set of equations needed to calculate $\hat{\Delta}(\omega+\mathrm{i} \gamma)$. A calculation of the neoclassical radial transport of toroidal angular momentum for general geometry, required in the development of the vorticity equation, is performed in Appendix B. Section 6 introduces a set of convenient normalisations for the set of equations. In Section 7 we discuss an intermediate radial region needed to connect the solutions of this set of equations to the ideal MHD region where $\Delta^{\prime}$ is defined. Finally, we draw some conclusions in Section 8.

\section{The Gyro-kinetic Equation}

We describe the plasma species $j$ by the gyro-kinetic equation (Tang et al.1980):

$$
\begin{aligned}
& \left(-\mathrm{i} \omega+v_{\|} \boldsymbol{b} \cdot \nabla+\boldsymbol{v}_{d j} \cdot \nabla\right) g_{j}-\overline{\exp \left(-\mathrm{i} L_{j}\right) C_{j}\left(g_{j} \exp \left(\mathrm{i} L_{j}\right)\right)} \\
& =-\mathrm{i} \frac{e_{j} f_{0_{j}}}{T_{j}}\left(\omega-\omega_{*_{j}}^{T}\right)\left(\left(\Phi-v_{\|} A_{\|}\right) J_{0}\left(z_{j}\right)+\frac{v_{\perp}}{k_{\perp}} \frac{\delta B_{\|}}{B} J_{1}\left(z_{j}\right)\right) \text {. }
\end{aligned}
$$

where $\boldsymbol{b}$ is a unit vector along the magnetic field, $v_{\|}$is the particle velocity along the magnetic field, $\Phi$ is the perturbed electrostatic potential, $A_{\|}$is the perturbed parallel component of the vector potential, $\delta B_{\|}$is the parallel component of the perturbed magnetic field and we have written the perturbed distribution as

$$
\delta f_{j}=g_{j} \exp \left(\mathrm{i} L_{j}\right)-\frac{e_{j} \Phi}{T_{j}} f_{0 j}
$$

with perturbation time dependencies: $\exp (-\mathrm{i} \omega t)$. Here, $L_{j}=\boldsymbol{k} \times \boldsymbol{v}_{\perp} . \boldsymbol{b} / \Omega_{j}$, with $\boldsymbol{k}$ the wavenumber and $v_{\perp}$ the velocity perpendicular to the magnetic field, is the gyro-phase factor, the operation $\bar{A}$ is a gyro-phase average over the quantity $A, \quad J_{0,1}$ are Bessel functions of argument: $z_{j}=k_{\perp} v_{\perp} / \Omega_{j}$, $f_{0 j}=n_{j}(\chi)\left(m_{j} / 2 \pi T_{j}(\chi)\right)^{3 / 2} \exp \left(-m_{j} v^{2} / 2 T_{j}(\chi)\right)$ are Maxwell distributions, with $\chi$ the poloidal flux, and 


$$
\begin{gathered}
\omega_{*_{j}}^{T}=\omega_{*_{j}}\left(1+\eta_{j}\left(\frac{m_{j} v^{2}}{2 T_{j}}-\frac{3}{2}\right)\right), \omega_{*_{j}}=\ell \frac{T_{j}}{e_{j}} \frac{\partial \ln n_{j}}{\partial \chi}, \eta_{j}=\frac{\partial \ln T_{j}}{\partial \ln n_{j}}, \\
\boldsymbol{v}_{d j}=\frac{1}{\Omega_{j}} \boldsymbol{b} \times\left(\frac{v_{\perp}^{2} \nabla \ell n B}{2}+v_{\|}^{2} \boldsymbol{\kappa}\right), \Omega_{j}=\frac{e_{j} B}{m_{j}}, \boldsymbol{\kappa}=\boldsymbol{b} . \nabla . \boldsymbol{b}
\end{gathered}
$$

with $v$ the particle speed, $\Omega_{j}$ the cyclotron frequency of species $j, \boldsymbol{\kappa}$ the curvature vector, $\ell$ the toroidal mode number of the perturbations and all gradients are taken at constant $(\mu, v)$, with $\mu=m_{j} v_{\perp}^{2} / 2 B$ the magnetic moment, or $(\lambda, v)$ with $\lambda=2 \mu / m_{j} v^{2}$ (thus $v_{\|}=\sigma v \sqrt{1-\lambda B}$, with $\sigma=\operatorname{sign}\left(v_{\|}\right)$). If $\theta$ is defined so that the safety factor $q=\boldsymbol{B} . \nabla \varphi / \boldsymbol{B} . \nabla \theta$, where $\boldsymbol{B}=\nabla \nabla+\nabla \varphi \times \nabla \chi$, is a flux function, then

$$
\nabla_{\|}=\boldsymbol{b} \cdot \nabla \theta \frac{\partial}{\partial \theta}+\boldsymbol{b} \cdot \nabla \varphi \frac{\partial}{\partial \varphi}=\frac{I}{R^{2} B}\left(\frac{\partial}{\partial \varphi}+\frac{1}{q} \frac{\partial}{\partial \theta}\right)
$$

so if we let

$$
g_{j}(r, \theta, \varphi) \rightarrow g_{j}(r, \theta) e^{\mathrm{i}(\ell \varphi-m \theta)}
$$

etc., then

$$
\nabla_{\|} g \rightarrow \frac{I}{R^{2} B q}\left[\mathrm{i}(\ell q-m) g_{j}+\frac{\partial}{\partial \theta} g_{j}\right] e^{\mathrm{i}(\ell \varphi-m \theta)}
$$

where $\ell q-m=\ell q^{\prime} x ; \quad q^{\prime}=d q / d \chi$ with $x=\chi-\chi_{s}, \chi_{s}$ being the resonant surface where $m=\ell q\left(\chi_{s}\right)$; prime denotes a derivative with respect to $\chi$, or equivalently, $x$.

Thus

$$
\begin{array}{r}
\frac{I v_{\|}}{R^{2} B q}\left(\frac{\partial}{\partial \theta}+\mathrm{i} \ell q^{\prime} x\right) g_{j}+v_{d j} \cdot \nabla g_{j}-\mathrm{i} \omega g_{j}-\overline{\exp \left(-\mathrm{i} L_{j}\right) C_{j}\left(g_{j} \exp \left(\mathrm{i} L_{j}\right)\right)} \\
=-\mathrm{i} \frac{e_{j} f_{0 j}}{T_{j}}\left(\omega-\omega_{* j}^{T}\right)\left(\left(\Phi-v_{\|} A_{\|}\right) J_{0}\left(z_{j}\right)+\frac{v_{\perp}}{k_{\perp}} \frac{\delta B_{\|}}{B} J_{1}\left(z_{j}\right)\right)
\end{array}
$$

Assuming both species are magnetised with $k_{\perp} v_{\perp} / \Omega_{j}<<1$, we expand $\exp \left(\mathrm{i} L_{j}\right) \cong 1+\mathrm{i} L_{j}$ and the Bessel functions for small $z_{j}$. We also introduce $h_{j}$ : 


$$
g_{j}=\frac{e_{j} \Psi}{T_{j}} f_{0 j}\left(1-\frac{\omega_{* j}^{T}}{\omega}\right)+h_{j}
$$

where $A_{\|}=-(\mathrm{i} / \omega) \nabla_{\|} \Psi$, so that the parallel electric field, $E_{\|}$, is given by

$$
E_{\|}=-\frac{I}{R^{2} B q}\left(\frac{\partial}{\partial \theta}+\mathrm{i} \ell q^{\prime} x\right)(\Phi-\Psi) .
$$

Then the fundamental kinetic equations are

$$
\begin{aligned}
& \left.\frac{I v_{\|}}{R^{2} B q}\left(\frac{\partial}{\partial \theta}+\mathrm{i} \ell q^{\prime} x\right) h_{j}+v_{d j} . \nabla h_{j}-\mathrm{i} \omega h_{j}-C_{j}\left(h_{j}\right)-\overline{L_{j} C_{j}\left(g_{j} L_{j}\right.}\right) \\
& =-\mathrm{i} \frac{e_{j} f_{0_{j}}}{T_{j}}\left(\omega-\omega_{*_{j}}^{T}\right)\left(\Phi-\Psi+\frac{v_{\perp}^{2}}{2 \Omega_{j}} \delta B_{\|}\right)-\frac{e_{j} f_{0_{j}}}{T_{j}}\left(1-\frac{\omega_{*_{j}}^{T}}{\omega}\right) \boldsymbol{v}_{d j} \cdot \nabla \Psi
\end{aligned}
$$

We note that relations (2) and (8) imply the perturbed density $\tilde{n}_{j}$ is given by

$$
\tilde{n}_{j}=\frac{e_{j} \Psi}{T_{j}}\left(1-\frac{\omega_{*_{j}}}{\omega}\right) n_{j}-\frac{e_{j} \Phi}{T_{j}} n_{j}+\hat{n}_{j}
$$

where $\hat{n}_{j}$ is the leading contribution to the density from $h_{j}$.

The perturbed and equilibrium quasi-neutrality conditions,

$$
\tilde{n}_{e}=\tilde{n}_{i} \equiv \tilde{n} ; \quad n_{e}=n_{i}=n_{0},
$$

allow us to obtain

$$
\frac{\hat{n}_{e}}{n_{0}}=\frac{\tilde{n}}{n_{0}}-\frac{e \Phi}{T_{e}}+\frac{e \Psi}{T_{e}}\left(1-\frac{\omega_{*_{e}}}{\omega}\right) ; \quad \frac{\hat{n}_{i}}{n_{0}}=\frac{\tilde{n}}{n_{0}}+\frac{e \Phi}{T_{i}}-\frac{e \Psi}{T_{i}}\left(1+\frac{T_{i}}{T_{e}} \frac{\omega_{*_{e}}}{\omega}\right) .
$$

Similarly from eqns. (2) and (8) we can obtain the perturbed temperatures:

$$
\frac{\hat{T}_{e}}{T_{e}}=\frac{\tilde{T}_{e}}{T_{e}}-\frac{e \Psi}{T_{e}} \frac{\omega_{*} \eta_{e}}{\omega} ; \frac{\hat{T}_{i}}{T_{i}}=\frac{\tilde{T}_{i}}{T_{i}}-\frac{e \Psi}{T_{e}} \frac{\omega_{* e} \eta_{i}}{\omega} .
$$


where $\hat{T}_{i}=\left(2 T_{j} / 3\right) \int \mathrm{d}^{3} v\left(m_{j} v^{2} / 2 T_{j}-3 / 2\right) h_{\mathrm{j}}$ is the contribution from $h_{j}$ etc.

\section{The Ion and Electron Solutions}

\section{(a) The Ion Solution}

We consider the 'collisional' case: $v_{i i}>\omega$. Introducing the proton charge, $e$, we have

$$
\begin{aligned}
& \frac{I v_{\|}}{R^{2} B q}\left(\frac{\partial}{\partial \theta}+\mathrm{i} \ell q^{\prime} x\right) h_{i}+v_{d i} \nabla h_{i}-\mathrm{i} \omega h_{i}-C_{i}\left(h_{i}\right)-\overline{L_{i} C_{i}\left(g_{i} L_{i}\right)} \\
& =-\mathrm{i} \frac{e f_{0 i}}{T_{i}}\left(\omega-\omega_{*_{i}}^{T}\right)\left(\Phi-\Psi+\frac{v_{\perp}^{2}}{2 \Omega_{i}} \delta B_{\|}\right)-\frac{e f_{0 i}}{T_{i}}\left(1-\frac{\omega_{* i}^{T}}{\omega}\right) v_{d i} \nabla \Psi
\end{aligned}
$$

We solve this equation by introducing an ordering scheme in terms of a small parameter, $\varepsilon_{i}$, where $\omega_{d r i} \sim v_{i} \sim \varepsilon_{i} \omega_{b i} ; \omega \sim \omega_{d r \theta} \sim \varepsilon_{i}^{2} \omega_{b i}$; we also order $z_{i} \sim \varepsilon_{i}$. Although we shall later assume a small number of trapped particles, $f_{t}<<1$, we do not order it in $\varepsilon_{i}$. The radial magnetic drift frequency, $\omega_{d r i}$, exceeds the azimuthal drift frequency, $\omega_{d r \theta}$, because of the narrow radial width of the semi-collisional layer.

Writing $h_{i}=h_{0 i}+\varepsilon_{i} h_{1 i}+\varepsilon_{i}^{2} h_{2 \mathrm{i}}+\ldots$, the lowest order solution satisfies

$$
\frac{\partial h_{0 i}}{\partial \theta}=0 \Rightarrow h_{0 \mathrm{i}}=h_{0 i}(v, \lambda, x, \sigma)
$$

while in first order we obtain

$$
\frac{I v_{\|}}{R^{2} B q} \frac{\partial}{\partial \theta} h_{1 i}=-v_{d r i} \frac{\partial}{\partial x} h_{0 i}+C_{i}\left(h_{0 i}\right)-\frac{e f_{0 i}}{T_{i}}\left(1-\frac{\omega_{* i}^{T}}{\omega}\right) v_{d r i} \frac{\partial}{\partial x} \psi
$$

where we note

$$
v_{d r j}=v_{d r j} \cdot \nabla \chi=\frac{I v_{\|}}{R^{2} B q} \frac{\partial}{\partial \theta}\left(\frac{I v_{\|}}{\Omega_{j}}\right)
$$

Thus $h_{1 i}$ for passing particles can be annihilated by applying the operation: $\left\langle B(\ldots) / v_{\|}\right\rangle$: 


$$
\langle\ldots\rangle=\oint \frac{(\ldots) \mathrm{d} \theta}{\boldsymbol{B} . \nabla \theta} / \oint \frac{\mathrm{d} \theta}{\boldsymbol{B} . \nabla \theta}=\oint(\ldots) R^{2} \mathrm{~d} \theta / \oint R^{2} \mathrm{~d} \theta
$$

since

$$
\frac{1}{\boldsymbol{B} . \nabla \theta}=\frac{q}{\boldsymbol{B} . \nabla \varphi}=\frac{q R^{2}}{I}
$$

For trapped particles we integrate along the bounce orbit, summing over $\sigma$ in the usual way, to obtain the constraint

$$
\left\langle\frac{B}{v_{\|}} C_{i}\left(h_{0 i}\right)\right\rangle=0
$$

where the integration is now between bounce points. This determines $h_{0 i}$, yielding:

$$
h_{0 i}=\left[\frac{\hat{n}_{i}}{n_{0}}+\left(u^{2}-\frac{3}{2}\right) \frac{\hat{T}_{i}}{T_{i}}\right] f_{0 i}
$$

where $u^{2}=m_{j} v^{2} / 2 T_{j}$ with $j=i$.

The equation for $h_{1 i}$ can then be integrated to give

$$
h_{1 i}=-\frac{I v_{\|}}{\Omega_{i}}\left[\frac{e}{T_{i}}\left(1-\frac{\omega_{*_{i}}^{T}}{\omega}\right) \Psi^{\prime} f_{0 i}+h_{0 i}^{\prime}\right]+H_{1 i}(v, \lambda, x, \sigma)
$$

where $H_{1 i}$ remains to be determined. This can be rewritten as

$$
\begin{gathered}
h_{1 i}=\frac{I v_{\|}}{\Omega_{i}}\left[A_{1}^{(i)}+\left(u^{2}-\frac{5}{2}\right) A_{2}^{(i)}\right] f_{0 i}+H_{1 i}(v, \lambda, x, \sigma), \\
A_{1}^{(i)}=-\frac{e \Psi^{\prime}}{T_{i}}\left(1-\frac{\omega_{*_{i}}}{\omega}\left(1+\eta_{i}\right)\right)-\left(\frac{\hat{n}_{i}^{\prime}}{n_{0}}+\frac{\hat{T}_{i}^{\prime}}{T_{i}}\right) \equiv-\left(\frac{\tilde{p}_{i}^{\prime}}{p_{0 i}}+\frac{e \Phi^{\prime}}{T_{i}}\right), \\
A_{2}^{(i)}=-\left(-\frac{\omega_{* i} \eta_{i}}{\omega} \frac{e \Psi^{\prime}}{T_{i}}+\frac{\hat{T}_{i}^{\prime}}{T_{i}}\right) \equiv-\frac{\tilde{T}_{i}^{\prime}}{T_{i}},
\end{gathered}
$$

where $p_{0 i}=n_{0} T_{i}$, on using eqns. (13) and (14). Note that the quantities $\tilde{p}_{i}^{\prime}$ and $\tilde{T}_{i}^{\prime}$ thus defined, depend on $\omega$. 
The equation for $h_{2 i}$ is

$$
\frac{v_{\|} I}{R^{2} B q} \frac{\partial}{\partial \theta} h_{2 i}+\frac{v_{\|} I}{R^{2} B q} \frac{\partial}{\partial \theta}\left(\frac{I v_{\|}}{\Omega_{i}}\right) \frac{\partial h_{1 i}}{\partial x}=C_{i i}\left(h_{1 i}\right)+C_{i e}\left(h_{1 i}\right)
$$

(where, for most purposes, we can ignore the small ion - electron collisional term, but see Section 7 later), which provides the constraint

$$
\left\langle\frac{B}{v_{\|}} C_{i i}\left(h_{1 i}\right)\right\rangle=0
$$

For the ions we take the model pitch-angle scattering collision operator that conserves momentum (Rutherford et al. 1970):

$$
\begin{gathered}
C_{i i}(h)=v_{i i}(u)\left(L(h)+\frac{m_{i}}{T_{i}} v_{\|} U_{\| i}^{*}(h) f_{0 i}\right), L(h)=\frac{2 v_{\|}}{v^{2} B} \frac{\partial}{\partial \lambda}\left(\lambda v_{\|} \frac{\partial}{\partial \lambda} h\right), \\
\left.U_{\| i}^{*}(h)=\int \mathrm{d}^{3} \boldsymbol{v} v_{i i}(u) v_{\|} h / \int d^{3} \boldsymbol{v} f_{0 i} v_{i i}(u)\right) m_{i} v_{\|}^{2} / 2 T_{i}, v_{i i}(u)=v_{0 i} \frac{\varphi(u)}{u^{3}}, \\
v_{0 i}=\frac{n_{0} e^{4} \ln \Lambda}{4 \pi \varepsilon_{0}^{2} m_{i}^{2}}\left(\frac{m_{i}}{2 T_{i}}\right)^{3 / 2}, \quad \varphi(u)=\left(1-\frac{1}{2 u^{2}}\right) \eta(u)+\frac{\eta^{\prime}(u)}{2 u}, \eta(u)=\frac{2}{\sqrt{\pi}} \int_{0}^{u} \mathrm{~d} t e^{-t^{2}}
\end{gathered}
$$

The constraint (26) yields an expression for $H_{1 i}$ and we find:

$$
h_{1 i}=\frac{I}{\Omega_{i}}\left(v_{\|}-H \hat{V}_{\|} \frac{B}{B_{0}}\right)\left(A_{1}^{(i)}+\left(u^{2}-\frac{5}{2}\right) A_{2}^{(i)}\right) f_{0 i}+\frac{m_{i}}{T_{i}}\left\langle\frac{B}{B_{0}} U_{\| i}^{*}\right\rangle H \hat{V}_{\|} f_{0 i},
$$

where $H\left(\lambda-\lambda_{c}\right)$ is the Heaviside function, and

$$
\hat{V}_{\|}=\frac{\sigma v^{2} B_{0}}{2} \int_{\lambda}^{\lambda_{c}} \frac{\mathrm{d} \lambda^{\prime}}{\left|\left\langle v_{\|}\right\rangle\right|}, \quad \lambda_{c}=\frac{B_{0}}{B_{M a x}} .
$$


Using eqn. (28) and integrating over velocity space:

$$
\int(\ldots) \mathrm{d}^{3} \boldsymbol{v}=\sum_{\sigma} \int(\ldots) \frac{B \pi v^{3} \mathrm{~d} v \mathrm{~d} \lambda}{\left|v_{\|}\right|},
$$

we can calculate $U_{\| i}^{*}$ self-consistently. Thus

$$
U_{\| i}^{*}=\frac{I}{\Omega_{i}} \frac{T_{i}}{m_{i}}\left(1-f_{c} \frac{B^{2}}{\left\langle B^{2}\right\rangle}\right)\left[A_{1}^{(i)}+\left(\frac{\left\{u^{2} v_{i i}\right\}}{\left\{v_{i i}\right\}}-\frac{5}{2}\right) A_{2}^{(i)}\right]+f_{c} B \frac{\left\langle B U_{\| i}^{*}\right\rangle}{\left\langle B^{2}\right\rangle},
$$

where we have defined the symbol $\{. . .$.$\} by$

$$
\{w\}=\int \mathrm{d}^{3} \boldsymbol{v} w\left(\frac{m v^{2}}{2 T}\right) \frac{m v_{\|}^{2}}{T} \frac{f_{0}}{n}=\frac{8}{3 \sqrt{\pi}} \int_{0}^{\infty} \mathrm{d} u w(u) u^{4} e^{-u^{2}}
$$

and

$$
\int \mathrm{d}^{3} \boldsymbol{v} w\left(\frac{m v^{2}}{2 T}\right) \frac{m v_{\|} \hat{V}_{\|}}{T} \frac{B}{B_{0}} H \frac{f_{0}}{n}=f_{c} \frac{B^{2}}{B_{0}^{2}}\{w\}
$$

where $f_{c}=1-f_{t}$, with $f_{t}$ the trapped particle fraction defined by

$$
f_{t}=1-\frac{3}{4}\left\langle B^{2}\right\rangle \int_{0}^{\lambda_{c}} \frac{\lambda \mathrm{d} \lambda}{\langle\sqrt{1-\lambda B}\rangle}
$$

Solving self-consistently for $\left\langle B U_{\| i}^{*}\right\rangle$, substituting for $A_{1}^{(i)}$ and $A_{2}^{(i)}$ from eqn. (24) and using the values of the collisional integrals from Appendix A, we obtain:

$$
\left\langle B U_{\| i}^{*}\right\rangle=-\frac{I T_{i}}{e}\left[\frac{\tilde{p}_{i}^{\prime}}{p_{0 i}}+\frac{e \Phi^{\prime}}{T_{i}}-1.17 \frac{\tilde{T}_{i}^{\prime}}{T_{i}}\right] .
$$


Then one can calculate the ion parallel flux from eqn. (28), to give

$$
U_{\| i}=-\frac{I T_{i}}{e B}\left(\frac{\tilde{p}_{i}^{\prime}}{p_{0 i}}+\frac{e \Phi^{\prime}}{T_{i}}-1.17 f_{c} \frac{B^{2}}{\left\langle B^{2}\right\rangle} \frac{\tilde{T}_{i}^{\prime}}{T_{i}}\right) \quad .
$$

It remains to address the determination of $\hat{n}_{i}$ and $\hat{T}_{i} ; \hat{n}_{i}$ is already given in terms of $\tilde{n}$ by eqn. (13), whereas $\hat{T}_{i}$ is obtained by applying the operator $\int(\ldots)\left(m_{i} v^{2} / 2\right) \mathrm{d}^{3} \boldsymbol{v}$ to the next order equation,

$$
\begin{gathered}
\frac{v_{\|} I}{R^{2} B q} \frac{\partial}{\partial \theta} h_{3 i}+\frac{v_{\|} I}{R^{2} B q} \frac{\partial}{\partial \theta}\left(\frac{I v_{\|}}{\Omega_{i}}\right) \frac{\partial h_{2 i}}{\partial x}+v_{d \theta i} . \nabla h_{0 i}-\mathrm{i} \omega h_{0 i}-C_{i}(h)-\overline{L_{i} C_{i}\left(g_{0 i} L_{i}\right)} \\
=-\frac{\mathrm{i} e}{T_{i}} f_{0 i}\left(\omega-\omega_{* i}^{T}\left(\Phi-\Psi+\frac{v_{\perp}^{2}}{2 \Omega_{i}} \delta B_{\|}\right)\right.
\end{gathered},
$$

since it annihilates the first term on the left by means of the flux surface average and the collision term, $C_{i}\left(h_{2 i}\right)$, due to its conservation properties. This will be elaborated in Section 4 (b).

\section{(b) The Electron Solution}

For the electrons, eqn. (10) becomes

$$
\begin{aligned}
\frac{I v_{\|}}{R^{2} B q}\left(\frac{\partial}{\partial \theta}+\mathrm{i} \ell q^{\prime} x\right) h_{e}+v_{d e} \cdot \nabla h_{e}-\mathrm{i} \omega h_{e}-C_{e}\left(h_{e}\right)-\overline{L_{e} C_{e}\left(g_{e} L_{e}\right)} \\
=\mathrm{i} \frac{e f_{0 e}}{T_{j}}\left(\omega-\omega_{*_{e}}^{T}\right)\left(\Phi-\Psi+\frac{v_{\perp}^{2}}{2 \Omega_{e}} \delta B_{\|}\right)+\frac{e f_{0 e}}{T_{e}}\left(1-\frac{\omega_{* e}^{T}}{\omega}\right) v_{d e} \nabla \Psi
\end{aligned}
$$

We employ a related ordering scheme to that of the ions in order to solve eqn. (38) for the electrons, introducing another small parameter, $\varepsilon_{e}$, where $\omega_{\text {dre }} \sim v_{e} \sim \varepsilon_{e} \omega_{b i} ; \omega \sim \omega_{\text {dre } \theta} \sim \varepsilon_{e}^{2} \omega_{b e}$. However, the semi-collisional 
electron model requires the additional ordering: $k_{\|} v_{t h e} \sim \varepsilon_{e}^{2} v_{t h e} / L_{c}$, where $k_{\|}=\ell q^{\prime} x / R q$. With $h_{e}=h_{0 e}+\varepsilon_{e} h_{1 e}+\varepsilon_{e}^{2} h_{2 e}+\ldots$,

the lowest order equation is

$$
\frac{\partial h_{0 e}}{\partial \theta}=0 \Rightarrow h_{0 e}=h_{0 e}(v, \lambda, x, \sigma)
$$

while in first order we obtain

$$
\frac{I v_{\|}}{R^{2} B q} \frac{\partial}{\partial \theta} h_{1 e}=-v_{d r e} \frac{\partial}{\partial x} h_{0 e}+C_{e}\left(h_{0 e}\right)+\frac{e f_{0 e}}{T_{e}}\left(1-\frac{\omega_{\omega_{e}}^{T}}{\omega}\right) v_{d r e} \frac{\partial}{\partial x} \Psi
$$

As in the case of the ions, $h_{1 e}$ can be annihilated for passing particles by applying the operation: $\left\langle B(. ..) v_{\|}\right\rangle$. For trapped particles we again integrate along the bounce orbit, summing over $\sigma$ in the usual way. Thus we obtain the constraint

$$
\left\langle\frac{B}{v_{\|}} C_{e}\left(h_{0 e}\right)\right\rangle=0
$$

which determines $h_{0 e}$, yielding:

$$
h_{0 e}=\left[\frac{\hat{n}_{e}}{n_{0}}+\left(u^{2}-\frac{3}{2}\right) \frac{\hat{T}_{e}}{T_{e}}\right] f_{0 e}
$$

where now $u^{2}=m_{e} v^{2} / 2 T_{e}$. The energy exchange term in the electron-ion collision operator is neglected (see eqn. (47) below), which makes the perturbation in the electron temperature independent of that in the ion temperature.

The equation for $h_{1 e}$ can then be integrated to give

$$
h_{1 e}=\frac{I v_{\|}}{\Omega_{e}}\left[\frac{e}{T_{e}}\left(1-\frac{\omega_{* e}^{T}}{\omega}\right) \Psi^{\prime} f_{0 e}-h_{0 e}^{\prime}\right]+H_{1 e}(v, \lambda, x, \sigma)
$$

where the function $H_{1 e}$ remains to be determined. This result can be rewritten as 


$$
\begin{gathered}
h_{1 e}=\frac{I v_{\|}}{\Omega_{e}}\left[A_{1}^{(e)}+\left(u^{2}-\frac{5}{2}\right) A_{2}^{(e)}\right] f_{0 \mathrm{e}}+H_{1 e}(v, \lambda, x, \sigma), \\
A_{1}^{(e)}=\frac{e \Psi^{\prime}}{T_{e}}\left(1-\frac{\omega_{*_{e}}}{\omega}\left(1+\eta_{e}\right)\right)-\left(\frac{\hat{n}_{e}^{\prime}}{n_{0}}+\frac{\hat{T}_{e}^{\prime}}{T_{e}}\right) \equiv-\left(\frac{\tilde{p}_{e}^{\prime}}{p_{0 e}}-\frac{e \Phi^{\prime}}{T_{e}}\right), \\
A_{2}^{(e)}=-\left(\frac{\omega_{*_{e}} \eta_{e}}{\omega} \frac{e \Psi^{\prime}}{T_{e}}+\frac{\hat{T}_{e}^{\prime}}{T_{e}}\right) \equiv-\frac{\tilde{T}_{e}^{\prime}}{T_{e}},
\end{gathered}
$$

with $p_{0 e}=n_{0} T_{e}$, where again we have used eqns. (13) and (14).

The next order equation is

$$
\frac{v_{\|} I}{R^{2} B q} \frac{\partial}{\partial \theta} h_{2 e}+\frac{v_{\|} I}{R^{2} B q} \frac{\partial}{\partial \theta}\left(\frac{I v_{\|}}{\Omega_{e}}\right) \frac{\partial h_{1 e}}{\partial x}+\frac{\mathrm{i} v_{\|} I \ell q^{\prime} x}{q R^{2} B} h_{0 e}=C_{e}\left(h_{1 e}\right),
$$

leading to the constraint

$$
\left\langle\frac{B}{v_{\|}} C_{e}\left(h_{1 e}\right)\right\rangle=\frac{\mathrm{i} I \ell q^{\prime} x}{q}\left\langle\frac{1}{R^{2}}\right\rangle h_{0 e}
$$

which determines $H_{1 e}(v, \lambda, x, \sigma)$.

The electron collision operator takes the form

$$
C_{e}(h)=C_{e e}(h)+v_{e i}(u)\left(L(h)+\frac{m_{e}}{T_{e}} v_{\|} U_{\| i} f_{0 e}\right)
$$

where $C_{e e}(h)$ is the electron-electron collision operator, $U_{\| i}$ is the ion bulk parallel velocity, $v_{e i}(u)=v_{0 e} / u^{3}$ with $v_{0 e}=n_{0} e^{4} \ln \Lambda\left(m_{e} / 2 T_{e}\right)^{3 / 2} /\left(4 \pi \varepsilon_{0}^{2} m_{e}^{2}\right)$.

At this point we introduce the two Spitzer functions (Spitzer \& Harm 1953): $h_{e s}^{(1)}$ and $h_{e s}^{(2)}$, where

$$
C_{e}\left(h_{e s}^{(1)}\right)=v_{\|} \frac{\mathrm{i} I \ell q^{\prime} x}{R^{2} B q}\left(\frac{\hat{n}_{e}}{n_{0}}+\frac{\hat{T}_{e}}{T_{e}}\right) f_{0 e} ; \quad C_{e}\left(h_{e s}^{(2)}\right)=v_{\|} \frac{\mathrm{i} I \ell q^{\prime} x}{R^{2} B q}\left(u^{2}-\frac{5}{2}\right) \frac{\hat{T}_{e}}{T_{e}} f_{0 e},
$$

so that the constraint equation becomes

$$
\left\langle\frac{B}{v_{\|}} C_{e}\left(h_{1 e}-h_{e s}\right)\right\rangle=0,
$$


with $h_{e s}=h_{e s}^{(1)}+h_{e s}^{(2)}$. Now that the collision operator is acting on a localised (to the trapped and barely passing region) distribution function we can use a model pitch-angle scattering operator for the electron-electron collisions (Rutherford et al. 1970).

$$
\begin{gathered}
C_{e e}(h)=v_{e e}(u)\left(L(h)+\frac{m_{e}}{T_{e}} v_{\|} U_{\| e}^{*}(h) f_{0 e}\right) \\
U_{\| e}^{*}(h)=\int \mathrm{d}^{3} \boldsymbol{v} v_{e e}(u) v_{\|} h / \int \mathrm{d}^{3} \boldsymbol{v} f_{0 e} v_{e e}(u) m_{e} v_{\|}^{2} / 2 T_{e}, \\
v_{e e}(u)=v_{0 e} \frac{\varphi(u)}{u^{3}}
\end{gathered}
$$

To remove the ion flow we write

$$
h_{e s}^{(1)}=h_{s}^{(1)}+\frac{m_{e} v_{\|}}{T_{e}} U_{\| i} f_{0 e}, h_{e s}^{(2)}=h_{s}^{(2)} .
$$

The functions $h_{s}^{(1,2)}$ are given by (Cohen et al. 1950, Spitzer \& Harm 1953):

$$
\begin{gathered}
h_{s}^{(1)}=\frac{v_{\|}}{R^{2} B} S^{(1)} \frac{D^{(1)}(u)}{u} f_{0 e}, \quad h_{s}^{(2)}=\frac{v_{\|}}{R^{2} B} S^{(2)} \frac{D^{(2)}(u)}{u} f_{0 e}, \\
S^{(1)}=\frac{4 \mathrm{i} \tau_{e i}}{3 \sqrt{\pi}} \frac{I \ell q^{\prime} x}{q}\left(\frac{\hat{n}_{e}}{n_{0}}+\frac{\hat{T}_{e}}{T_{e}}\right) \equiv \frac{4 \mathrm{i} \tau_{e i}}{3 \sqrt{\pi}} \frac{I q^{\prime} x}{q}\left(\frac{\tilde{p}_{e}}{p_{0 e}}-\frac{e(\Phi-\Psi)}{T_{e}}-\frac{\omega_{* e}}{\omega} \frac{e \Psi}{T_{e}}\right) \\
S^{(2)}=\frac{4 \mathrm{i} \tau_{e i}}{3 \sqrt{\pi}} \frac{I \ell q^{\prime} x}{q} \frac{\hat{T}_{e}}{T_{e}} \equiv \frac{4 \mathrm{i} \tau_{e i}}{3 \sqrt{\pi}} \frac{I \ell q^{\prime} x}{q}\left(\frac{\tilde{T}_{e}}{T_{e}}-\frac{\omega_{* e} \eta_{e}}{\omega} \frac{e \Psi}{T_{e}}\right)
\end{gathered}
$$

where $\tau_{e i}=3 \sqrt{\pi} / 4 v_{0 e}$ and $D^{(1)}$ and $D^{(2)}$ are related to the normalised responses to a parallel electric field and thermal force, tabulated in Spitzer and Harm (Spitzer \& Harm 1953) (to be precise, $D^{(1)}=-D / A$ and $D^{(2)}=2 D / B$ as given in Tables I and II of Spitzer \& Harm 1953)

So, on defining $v_{e}=v_{e e}+v_{e i}$, the constraint equation becomes 


$$
\left\langle\frac{B}{v_{\|}}\left[v_{e} L\left(h_{1 e}-h_{s}\right)+\frac{m_{e} v_{\|}}{T_{e}}\left(v_{e e} U_{\| e}^{*}\left(h_{1 e}-h_{s}\right)+v_{e i} U_{\| i}\right) f_{0 e}\right]\right\rangle=0
$$

where $h_{s}=h_{s}^{(1)}+h_{s}^{(2)}$. Thus

$$
\begin{aligned}
& \frac{\partial}{\partial \lambda}\left(\lambda\left\langle v_{\|}\right\rangle\right.\left.\frac{\partial}{\partial \lambda} H_{1 e}\right)=\frac{I B_{0} v^{2}}{2 \Omega_{e}}\left(A_{1}^{(e)}+\left(u^{2}-\frac{5}{2}\right) A_{2}^{(e)}\right) f_{0 e} \\
&-\frac{m_{e} v^{2}}{2 T_{e}}\left\langle\frac{B}{v_{e}}\left(v_{e e} U_{\| e}^{*}+v_{e i} U_{\| i}\right) f_{0 e}\right\rangle-\frac{\left(S^{(1)} D^{(1)}(u)+S^{(2)} D^{(2)}(u)\right) v^{2}}{2 u}\left\langle\frac{1}{R^{2}}\right\rangle f_{0 e}
\end{aligned}
$$

Integrating eqn. (54) in $\lambda$, we find

$$
\begin{aligned}
h_{1 e}=\frac{I}{\Omega_{e}}\left(v_{\|}\right. & \left.-H \hat{V}_{\|} \frac{B}{B_{0}}\right)\left(A_{1}^{(e)}+\left(u^{2}-\frac{5}{2}\right) A_{2}^{(e)}\right) f_{0 e} \\
& +\left[\frac{m_{e}}{T_{e}}\left\langle\frac{B}{B_{0} v_{e}}\left(v_{e e} U_{\| e}^{*}+v_{e i} U_{\| i}\right)\right)+\frac{\left(S^{(1)} D^{(1)}(u)+S^{(2)} D^{(2)}(u)\right)}{B_{0} u}\left\langle\frac{1}{R^{2}}\right\rangle\right] H \hat{V}_{\|} f_{0 \mathrm{e}}
\end{aligned}
$$

Using the definition of $U_{\| e}^{*}$ in eqn. (50) and integrating over velocity space, we can calculate $U_{\| e}^{*}\left(h_{1 e}-h_{s}\right)$ self-consistently. However, as argued by Helander and Sigmar (Helander \& Sigmar 2002), $\left\langle B U_{\| e}^{*}\right\rangle=\left\langle B U_{\| i}\right\rangle+O\left(f_{t}\right)$, provided $f_{t}<<O\left(v_{e i} / v_{e}\right)$. Thus result (55) simplifies to

$$
\begin{aligned}
h_{1 e}=\frac{I}{\Omega_{e}}\left(v_{\|}-\right. & \left.H \hat{V}_{\|} \frac{B}{B_{0}}\right)\left(A_{1}^{(e)}+\left(u^{2}-\frac{5}{2}\right) A_{2}^{(e)}\right) f_{0 e} \\
& +\left[\frac{m_{e}\left\langle B U_{\| i}\right\rangle}{T_{e}}+\frac{\left(S^{(1)} D^{(1)}(u)+S^{(2)} D^{(2)}(u)\right)}{u}\left\langle\frac{1}{R^{2}}\right\rangle\right] \frac{H \hat{V}_{\|}}{B_{0}} f_{0 e}
\end{aligned}
$$


It remains to determine $\hat{n}_{e}$ and $\hat{T}_{e}$; these are obtained in Section 4(b) by applying the operators $\left\langle\int(\ldots) \mathrm{d}^{3} \boldsymbol{v}\right\rangle$ and $\int(\ldots)\left(m_{e} v^{2} / 2\right) \mathrm{d}^{3} \boldsymbol{v}$ to the next order equation:

$$
\begin{aligned}
& \frac{v_{\|} I}{R^{2} B q} \frac{\partial}{\partial \theta} h_{3 e}+\frac{v_{\|} I}{R^{2} B q} \frac{\partial}{\partial \theta}\left(\frac{I v_{\|}}{\Omega_{e}}\right) \frac{\partial h_{2 e}}{\partial x}+\frac{\mathrm{i} I \ell q^{\prime} x}{q R^{2} B} v_{\|} h_{1 e}+v_{d \theta e} \cdot \nabla h_{0 e} \\
& -\mathrm{i} \omega h_{0 e}-C_{e}\left(h_{2 e}\right)-\overline{L_{e} C_{e}\left(g_{0 e} L_{e}\right)}=\frac{\mathrm{i} e}{T_{e}} f_{0 e}\left(\omega-\omega_{* e}^{T}\right)\left(\Phi-\Psi+\frac{v_{\perp}^{2}}{2 \Omega_{e}} \delta B_{\|}\right)
\end{aligned}
$$

\section{The Perturbed Density and Temperatures}

In this Section we derive equations for the perturbed electron density and temperature and ion temperature in terms of the perturbed potentials $\Phi$ and $\Psi$. The ion density perturbation can be obtained from the leading order quasineutrality condition, see eqn. (12).

The other perturbed field that these quantities depend on, is $\delta B_{\|}$, which is obtained from the perpendicular component of Ampère's law. The perpendicular current can be calculated from the first order in a Larmor radius expansion of the distribution function (2), again recalling eqns. (8), (22) and (42). Introducing this result into the perpendicular component of Ampère's law yields (Tang et al. 1980)

$$
\frac{\delta B_{\|}}{B}=-\frac{\mu_{0} \tilde{p}}{B^{2}} ; \quad \tilde{p}=\tilde{n}\left(T_{e}+T_{i}\right)+n_{0}\left(\widetilde{T}_{e}+\widetilde{T}_{i}\right) .
$$


In Section 5 we will discuss the parallel component of Ampère's law and find that in leading order, $\Psi$ is independent of the poloidal angle, $\theta$. Since $\hat{n}_{j}$ and $\hat{T}_{j}$ are also independent of $\theta$, it follows from eqns. (13) and (14) that $\tilde{n}_{j}, \tilde{T}_{j}$ and $\Phi$ are also independent of $\theta$.

\section{(a) The Perturbed Electron Density and Temperature}

As mentioned earlier, the determination of $\hat{n}_{e}$ and $\hat{T}_{e}$ is accomplished by applying the annihilators $\left\langle\int(\ldots) \mathrm{d}^{3} \boldsymbol{v}\right\rangle$ and $\int(\ldots)\left(m_{e} v^{2} / 2\right) \mathrm{d}^{3} v$ to the third order equation (57), which both eliminate $h_{3 e}$.

The first operation results in

$$
\begin{gathered}
\left.\left\langle\int \mathrm{d}^{3} \boldsymbol{v} \frac{v_{\|} I}{R^{2} B q} \frac{\partial}{\partial \theta}\left(\frac{I v_{\|}}{\Omega_{e}}\right) \frac{\partial h_{2 e}}{\partial x}\right\rangle+\left\langle\frac{\mathrm{i} \ell I q^{\prime} x}{R^{2} B q} \int \mathrm{d}^{3} \boldsymbol{v} v_{\|} h_{1 e}\right\rangle+\left\langle\int \mathrm{d}^{3} \boldsymbol{v} \boldsymbol{v}_{d \theta e} \cdot \nabla h_{0 e}\right\rangle-\left\langle\int \mathrm{d}^{3} \boldsymbol{v} \overline{L_{e} C_{e}\left(g_{0 e} L_{e}\right.}\right)\right\rangle \\
=\mathrm{i} \omega \hat{n}_{e}+\mathrm{i} n_{0}\left\langle\frac{e}{T_{e}}\left(\omega-\omega_{*_{e}}\right)(\Phi-\Psi)-\frac{\delta B_{\|}}{B}\left(\omega-\omega_{*_{e}}\left(1+\eta_{e}\right)\right)\right\rangle
\end{gathered}
$$

Here one can recognise the first three terms on the left-hand-side as representing, respectively, the surface-averaged contributions to the electron continuity equation of the divergences of the radial flux, $\Gamma_{r e}$ (which we will see below is related to Pfirsch-Schlüter and neoclassical radial transport), the parallel flux, 
$\Gamma_{\| e}$, and the 'azimuthal flux', $\Gamma_{e}^{A z}$ (Fitzpatrick 1989), while the fourth corresponds to the contribution from classical radial transport.

The second, the energy moment, takes the form

$$
\begin{aligned}
& \left\langle\int \mathrm{d}^{3} \boldsymbol{v} \frac{v_{\|} I}{R^{2} B q} \frac{\partial}{\partial \theta}\left(\frac{I v_{\|}}{\Omega_{e}}\right) \frac{m_{e} v^{2}}{2} \frac{\partial h_{2 e}}{\partial x}\right\rangle+\left\langle\frac{\mathrm{i} \ell I q^{\prime} x}{R^{2} B q} \iint \mathrm{d}^{3} v v_{\|} \frac{m_{e} v^{2}}{2} h_{1 e}\right\rangle \\
& +\left\langle\int \mathrm{d}^{3} \boldsymbol{v} \frac{m_{e} v^{2}}{2} v_{d \theta e} \cdot \nabla h_{0 e}\right\rangle-\left\langle\int \mathrm{d}^{3} \boldsymbol{v} \frac{m_{e} v^{2}}{2} \overline{L_{e} C_{e}\left(g_{0 e} L_{e}\right)}\right\rangle \\
& \quad=\frac{3}{2} \mathrm{i} \omega\left(\hat{n}_{e}+\frac{n_{0} \hat{T}_{e}}{T_{e}}\right)+\mathrm{i} n_{0}\left\langle\frac{3}{2} \frac{e}{T_{e}}\left(\omega-\omega_{*_{e}}\left(1+\eta_{e}\right)\right)(\Phi-\Psi)-\frac{5}{2} \frac{\delta B_{\|}}{B}\left(\omega-\omega_{*_{e}}\left(1+2 \eta_{e}\right)\right)\right\rangle
\end{aligned}
$$

with a similar interpretation in terms of fluxes.

It is helpful to separate the electron and ion distributions into a Pfirsch-Schlüterlike, $h_{e}^{P S}$, and a banana neoclassical part, $h_{e}^{\text {Ban }}$, and express the quantities $\hat{n}_{j}$ and $\hat{T}_{j}$ in terms of $\tilde{n}_{j}$ and $\tilde{T}_{j}$. Thus for the electrons we write

$$
h_{1 e}-h_{e s}=h_{e}^{P S}+h_{e}^{B a n}
$$

where: 


$$
\begin{aligned}
h_{e}^{P S}=\frac{I v_{\|} m_{e}}{e B}\left(1-\frac{B^{2}}{\left\langle B^{2}\right\rangle}\right)\left[\frac{\tilde{p}^{\prime}}{p_{0 e}}+\left(u^{2}-\frac{5}{2}\right) \frac{\tilde{T}_{e}^{\prime}}{T_{e}}\right] f_{0 e}+\frac{I v_{\|} m_{e}}{e B}\left(1-\frac{B^{2}}{\left\langle B^{2}\right\rangle}\right)\left[\frac{\tilde{p}_{i}^{\prime}}{p_{0 e}}+\frac{e \Phi^{\prime}}{T_{e}}\right] f_{0 e} \\
-\frac{4 \mathrm{i} \tau_{e i} v_{\|}}{3 \sqrt{\pi}} \frac{I \ell q^{\prime} x}{q}\left(\frac{1}{R^{2} B}-\frac{B}{\left\langle B^{2}\right\rangle}\left\langle\frac{1}{R^{2}}\right\rangle\right) \\
\quad \times\left\{\frac{D^{(1)}(u)}{u}\left[\frac{\tilde{p}_{e}}{p_{0 e}}-\frac{e(\Phi-\Psi)}{T_{e}}-\frac{\omega_{*_{e}}\left(1+\eta_{e}\right)}{\omega} \frac{e \Psi}{T_{e}}\right]+\frac{D^{(2)}(u)}{u}\left[\frac{\tilde{T}_{e}}{T_{e}}-\frac{\omega_{*_{e}} \eta_{e}}{\omega} \frac{e \Psi}{T_{e}}\right]\right\} f_{0 e}
\end{aligned}
$$

and:

$$
\begin{aligned}
h_{e}^{B a n}=\frac{I m_{e}}{e}\left(\frac{B}{\left\langle B^{2}\right\rangle} v_{\|}\right. & \left.-\frac{H \hat{V}_{\|}}{B_{0}}\right)\left[\frac{\tilde{p}^{\prime}}{p_{0 e}}+\left(u^{2}-\frac{5}{2}\right) \frac{\tilde{T}_{e}^{\prime}}{T_{e}}-1.17 \frac{\tilde{T}_{i}^{\prime}}{T_{e}}\right] f_{0 e}-\frac{4 \mathrm{i} \tau_{e i}}{3 \sqrt{\pi}} \frac{I \ell q^{\prime} x}{q}\left(\frac{B}{\left\langle B^{2}\right\rangle} v_{\|}-\frac{H \hat{V}_{\|}}{B_{0}}\right)\left\langle\frac{1}{R^{2}}\right\rangle \\
& \times\left\{\frac{D^{(1)}(u)}{u}\left[\frac{\tilde{p}_{e}}{p_{0 e}}-\frac{e(\Phi-\Psi)}{T_{e}}-\frac{\omega_{*_{e}}\left(1+\eta_{e}\right)}{\omega} \frac{e \Psi}{T_{e}}\right]+\frac{D^{(2)}(u)}{u}\left[\frac{\tilde{T}_{e}}{T_{e}}-\frac{\omega_{* e} \eta_{e}}{\omega} \frac{e \Psi}{T_{e}}\right]\right\} f_{0 e},
\end{aligned}
$$

where we have substituted for $U_{\| i}$ from eqn. (36).

Similarly, it is convenient to separate $h_{1 i}$ in eqn. (28) into Pfirsch-Schlüter, $h_{i}^{P S}$, and banana contributions, $h_{i}^{B a n}$, writing:

$$
h_{1 i}=h_{i}^{\text {Flow }}+h_{i}^{P S}+h_{i}^{\text {Ban }}
$$

where $h_{i}^{\text {Flow }}$ describes the mean flow:

$$
h_{i}^{\text {Flow }}=-\frac{I v_{\|} m_{i} B}{e\left\langle B^{2}\right\rangle}\left[\frac{\tilde{p}_{i}^{\prime}}{p_{0 i}}+\frac{e \Phi^{\prime}}{T_{i}}-1.17 \frac{\tilde{T}_{i}^{\prime}}{T_{i}}\right] f_{0 i}
$$

while 


$$
h_{i}^{P S}=-\frac{I v_{\|} m_{i}}{e B}\left(1-\frac{B^{2}}{\left\langle B^{2}\right\rangle}\right)\left[\frac{\tilde{p}_{i}^{\prime}}{p_{0 i}}+\frac{e \Phi^{\prime}}{T_{i}}+\left(u^{2}-\frac{5}{2}\right) \frac{\tilde{T}_{i}^{\prime}}{T_{i}}\right] f_{0 i}
$$

and

$$
h_{i}^{B a n}=-\frac{I m_{i}}{e}\left(\frac{B v_{\|}}{\left\langle B^{2}\right\rangle}-\frac{H \hat{V}_{\|}}{B_{0}}\right)\left(u^{2}-1.33\right) \frac{\tilde{T}_{i}^{\prime}}{T_{i}} f_{0 i}
$$

The first term represents a drifting Maxwellian and therefore does not contribute to the ion-ion collision term.

\section{(i) Electron Radial Fluxes}

First we consider the radial fluxes arising from classical transport. Recalling the definitions of $h_{0 j}$ and $L_{j}$ and noting the conservation of momentum in likeparticle collisions, we obtain

$$
\begin{aligned}
& \left\langle\int d^{3} v \overline{L_{e} C_{e}\left(g_{0 e} L_{e}\right)}\right\rangle \equiv \frac{\mathrm{d}}{\mathrm{d} r}\left\langle\Gamma_{r, e}^{C}\right\rangle \\
& =\frac{m_{e} T_{e}}{e^{2}} \frac{\mathrm{d}^{2}}{\mathrm{~d} x^{2}}\left\langle\frac{|\nabla \chi|^{2}}{B^{2}}\right\rangle \int \mathrm{d}^{3} v \frac{m_{e} v_{r}}{T_{e}} C_{e i}\left[v_{r}\left(\frac{\tilde{p}_{e}}{p_{0 e}}-\frac{e \Phi}{T_{e}}+\left(u^{2}-\frac{5}{2}\right) \frac{\tilde{T}_{e}}{T_{e}}\right)\right],
\end{aligned}
$$

where $r$ represents a 'radial-like' co-ordinate labelling flux surfaces, $v_{r}$ being the corresponding radial component of the velocity. We note the gyrocorrection to the scattering target ion distribution has the form

$$
v_{r} \frac{m_{i}}{e B}\left(\frac{\tilde{p}_{i}}{p_{0 i}}+\frac{e \Phi}{T_{i}}+\left(u^{2}-\frac{5}{2}\right) \frac{\tilde{T}_{i}}{T_{i}}\right) f_{0 i}
$$


The velocity integral can be evaluated by observing that it involves the matrix elements of the collision operator between Laguerre polynomials given by Helander and Sigmar (Section 4.5 of Helander \& Sigmar 2002). The resultant contribution from eqn. (68) is the familiar Braginskii expression:

$$
\frac{\mathrm{d}}{\mathrm{d} r}\left\langle\Gamma_{r, e}^{C}\right\rangle=-\frac{m_{e} T_{e}}{e^{2} \tau_{e i}}\left\langle\frac{|\nabla \chi|^{2}}{B^{2}}\right\rangle \frac{\mathrm{d}^{2}}{\mathrm{~d} x^{2}}\left(\frac{\tilde{p}}{p_{0 e}}-\frac{3}{2} \frac{\tilde{T}_{e}}{T_{e}}\right) n_{0}
$$

A similar calculation for the classical heat flux results in

$$
\frac{\mathrm{d}}{\mathrm{d} r}\left\langle q_{r, e}^{C}\right\rangle=-\frac{m_{e} T_{e}}{e^{2} \tau_{e i}}\left\langle\frac{|\nabla \chi|^{2}}{B^{2}}\right\rangle \frac{\mathrm{d}^{2}}{\mathrm{~d} x^{2}}\left(-\frac{3}{2} \frac{\tilde{p}}{p_{0 e}}+4.66 \frac{\tilde{T}_{e}}{T_{e}}\right) n_{0} T_{e}
$$

so that the classical energy flux, $\left\langle Q_{r, e}^{C}\right\rangle=\left\langle q_{r, e}^{C}\right\rangle+(5 / 2)\left\langle\Gamma_{r, e}^{C}\right\rangle$, satisfies

$$
\frac{\mathrm{d}}{\mathrm{d} r}\left\langle Q_{r, e}^{C}\right\rangle=-\frac{m_{e} T_{e}}{e^{2} \tau_{e i}}\left\langle\frac{|\nabla \chi|^{2}}{B^{2}}\right\rangle \frac{\mathrm{d}^{2}}{\mathrm{~d} x^{2}}\left(\frac{\tilde{p}}{p_{0 e}}+0.91 \frac{\tilde{T}_{e}}{T_{e}}\right) n_{0} T_{e}
$$

Turning to the leading term in eqn. (59), we can eliminate $h_{2 e}$ by integrating by parts in $\theta$ and using eqn. (45) to obtain

$$
\begin{aligned}
&\left\langle\int \mathrm{d}^{3} \boldsymbol{v} \frac{v_{\|} I}{R^{2} B q}\right.\left.\frac{\partial}{\partial \theta}\left(\frac{I v_{\|}}{\Omega_{e}}\right) h_{2 e}\right\rangle \equiv\left\langle\Gamma_{r, e}^{N C}\right\rangle \\
&=\left\langle\frac{I}{\Omega_{e}} \int \mathrm{d}^{3} \boldsymbol{v}\left[\frac{v_{\|}^{2} I}{R^{2} B q} \frac{\partial}{\partial \theta}\left(\frac{I}{\Omega_{e}}\right) \frac{\mathrm{d} h_{1 e}}{\mathrm{~d} x}+\frac{\mathrm{i} \ell I q^{\prime} x}{R^{2} B q} v_{\|}^{2} h_{0 e}-v_{\|} C_{e}\left(h_{1 e}\right)\right]\right\rangle
\end{aligned}
$$


where the first term vanishes if the equilibrium is up-down symmetric, which we take to be the case here. Thus, finally, we recognise this contribution as the divergence of the neoclassical radial flux

$$
\frac{\mathrm{d}}{\mathrm{d} r}\left\langle\Gamma_{r, e}^{N C}\right\rangle=-\frac{\mathrm{d}}{\mathrm{d} x}\left\langle\frac{I}{\Omega_{e}} \int \mathrm{d}^{3} \boldsymbol{v} v_{\|} C_{e}\left(h_{1 e}-h_{e s}^{(1)}-h_{e s}^{(2)}\right)\right\rangle
$$

Likewise, for the thermal equation, we find

$$
\frac{\mathrm{d}}{\mathrm{d} r}\left\langle Q_{r, e}^{N C}\right\rangle=-\frac{\mathrm{d}}{\mathrm{d} x}\left\langle\frac{I}{\Omega_{e}} \int \mathrm{d}^{3} \boldsymbol{v} v_{\|} \frac{m_{e} v^{2}}{2} C_{e}\left(h_{1 e}-h_{e s}^{(1)}-h_{e s}^{(2)}\right)\right\rangle
$$

To evaluate the velocity integrals in eqns. (74) and (75), we recall the result (33).

Considering the Pfirsch-Schlüter and banana contributions separately, we introduce their respective distribution functions. From momentum conservation it is again clear that only $e-i$ collisions contribute to eqn. (74) in both cases. For the Pfirsch-Schlüter contribution the calculation has the same structure as the classical case, with the substitution $v_{r} \rightarrow v_{\|}$in the distribution function. However, since we are now considering the difference $h_{1 e}-h_{e s}$, the scattering 'ion distribution' is effectively at rest when evaluating the collisional matrix elements. Recalling eqn. (62), the result is 


$$
\begin{aligned}
& \frac{1}{n_{0}} \frac{\mathrm{d}}{\mathrm{d} r}\left\langle\Gamma_{r, e}^{P S}\right\rangle=-\frac{m_{e} T_{e}}{e^{2} \tau_{e i}} I^{2}\left(\left\langle\frac{1}{B^{2}}\right\rangle-\frac{1}{\left\langle B^{2}\right\rangle}\right) \frac{\mathrm{d}^{2}}{\mathrm{~d} x^{2}}\left(\frac{\tilde{p}}{p_{0 e}}-\frac{3}{2} \frac{\tilde{T}_{e}}{T_{e}}\right) \\
& -\frac{\mathrm{i} I^{2} \ell q^{\prime} T_{e}}{e q}\left(\left\langle\frac{1}{R^{2} B^{2}}\right\rangle-\frac{1}{\left\langle B^{2}\right\rangle}\left\langle\frac{1}{R^{2}}\right\rangle\right) \frac{\mathrm{d}}{\mathrm{d} x}\left[x\left(\frac{\tilde{p}_{e}}{p_{0 e}}-\frac{e \Phi}{T_{e}}+\left(1-\frac{\omega_{*_{e}}}{\omega}\left(1+\eta_{e}\right)\right) \frac{e \Psi}{T_{e}}\right)\right]
\end{aligned}
$$

For the heat flux, we obtain

$$
\begin{aligned}
& \frac{1}{\mathrm{n}_{0} T_{e}} \frac{\mathrm{d}}{\mathrm{d} r}\left\langle Q_{r, e}^{P S}\right\rangle=-\frac{m_{e} T_{e} I^{2}}{e^{2} \tau_{e i}}\left(\left\langle\frac{1}{B^{2}}\right\rangle-\frac{1}{\left\langle B^{2}\right\rangle}\right) \frac{\mathrm{d}^{2}}{\mathrm{~d} x^{2}}\left(\frac{\tilde{p}}{p_{0 e}}+0.91 \frac{\tilde{T}_{e}}{T_{e}}\right) \\
& -\frac{\mathrm{i} I^{2} \ell q^{\prime} T_{e}}{e q}\left(\left\langle\frac{1}{R^{2} B^{2}}\right\rangle-\frac{1}{\left\langle B^{2}\right\rangle}\left\langle\frac{1}{R^{2}}\right\rangle\right) \frac{\mathrm{d}}{\mathrm{d} x}\left[\frac{5}{2} x\left(\frac{\tilde{p}_{e}}{p_{0 e}}-\frac{e \Phi}{T_{e}}+\frac{\tilde{T}_{e}}{T_{e}}+\frac{e \Psi}{T_{e}}\left(1-\frac{\omega_{*_{e}}}{\omega}\left(1+2 \eta_{e}\right)\right)\right)\right]
\end{aligned}
$$

Finally, we consider the banana contribution. For the classical and PfirschSchlüter contributions we used the exact collision operator, since the distributions were not localised in velocity space. However, the banana contribution to $h_{1 e}-h_{s s}$ is localised and it is sufficient to use the model collision operator (50), which leads to $\left\langle\boldsymbol{\Gamma}_{e}^{B a n} \cdot \nabla \chi\right\rangle=-\frac{I}{e}\left\langle\frac{m_{e}}{B} \int \mathrm{d}^{3} \boldsymbol{v} v_{\|} v_{e}\left(h_{1 e}-h_{s}^{(1)}-h_{s}^{(2)}-\frac{m_{e} v_{\|}}{T_{e} v_{e}}\left(v_{e e} U_{\| e}^{*}+v_{e i} U_{\| i}\right) f_{0 e}\right)^{B a n}\right\rangle$

Substituting expression (63), using result (36) and approximating $\left\langle B U_{\| e}^{*}\right\rangle \cong\left\langle B U_{\| i}\right\rangle$ as discussed below eqn. (55), we obtain 


$$
\begin{aligned}
& \frac{1}{\mathrm{n}_{0}} \frac{\mathrm{d}}{\mathrm{d} r}\left\langle\Gamma_{r, e}^{B a n}\right\rangle=-f_{t} \frac{I^{2} m_{e} T_{e}\left\{v_{e}\right\}}{e^{2}\left\langle B^{2}\right\rangle} \frac{\mathrm{d}^{2}}{\mathrm{~d} x^{2}}\left[\frac{\tilde{p}}{p_{0 e}}+\left(\frac{\left\{u^{2} v_{e}\right\}}{\left\{v_{e}\right\}}-\frac{5}{2}\right) \frac{\tilde{T}_{e}}{T_{e}}-1.17 \frac{\tilde{T}_{i}}{T_{e}}\right] \\
& +\frac{4 \tau_{e i} \mathrm{i} \ell q^{\prime}}{3 \sqrt{\pi} q} \frac{f_{t}}{\left\langle B^{2}\right\rangle}\left\langle\frac{I^{2}}{R^{2}}\right) \frac{T_{e}}{e} \frac{\mathrm{d}}{\mathrm{d} x}\left(x\left[\left(\frac{\tilde{p}_{e}}{p_{0 e}}-\frac{e(\Phi-\Psi)}{T_{e}}-\frac{\omega_{\omega_{e}}}{\omega}\left(1+\eta_{e}\right) \frac{e \Psi}{T_{e}}\right)\left\{\frac{v_{e} D^{(1)}}{u}\right\}+\left(\frac{\tilde{T}_{e}}{T_{e}}-\frac{\omega_{*_{e}} \eta_{e}}{\omega} \frac{e \Psi}{T_{e}}\right)\left\{\frac{v_{e} D^{(2)}}{u}\right\}\right]\right)
\end{aligned}
$$

Introducing the numerical values of the various collisional averages given by the integrals in Appendix A, this becomes:

$$
\begin{aligned}
& \frac{1}{n_{0}} \frac{\mathrm{d}}{\mathrm{dr}}\left\langle\Gamma_{r, e}^{B a n}\right\rangle=-1.53 f_{t} \frac{I^{2} m_{e} T_{e}}{e^{2}\left\langle B^{2}\right\rangle \tau_{e i}} \frac{\mathrm{d}^{2}}{\mathrm{~d} x^{2}}\left[\frac{\tilde{p}}{p_{0 e}}+1.39 \frac{\tilde{T}_{e}}{T_{e}}-1.17 \frac{\tilde{T}_{i}}{T_{e}}\right] \\
& -1.67 \frac{f_{t}}{\left\langle B^{2}\right\rangle}\left\langle\frac{I^{2}}{R^{2}}\right\rangle \frac{\mathrm{i} \ell q^{\prime} T_{e}}{e q} \frac{\mathrm{d}}{\mathrm{d} x}\left(x\left[\frac{\tilde{p}_{e}}{p_{0 e}}-\frac{e(\Phi-\Psi)}{T_{e}}-\frac{\omega_{x_{e}}}{\omega}\left(1+1.02 \eta_{e}\right) \frac{e \Psi}{T_{e}}+0.02 \frac{\tilde{T}_{e}}{T_{e}}\right]\right)
\end{aligned}
$$

Similarly, we find

$$
\begin{aligned}
& \left.\frac{1}{n_{0} T_{e}} \frac{\mathrm{d}}{\mathrm{d} r}\left\langle Q_{r e e}^{B a n}\right\rangle=-f_{t} \frac{I^{2} m_{e} T_{e}\left\{u^{2} v_{e}\right\}}{e^{2}\left\langle B^{2}\right\rangle} \frac{\mathrm{d}^{2}}{\mathrm{~d} x^{2}}\left[\frac{\tilde{p}}{p_{0 e}}+\left(\frac{\left\{u^{4} v_{e}\right\}}{\left\{u^{2} v_{e}\right.}\right\}-\frac{5}{2}\right) \frac{\tilde{T}_{e}}{T_{e}}+\left(\frac{\left\{u^{2} v_{i i}\right\}}{\left\{v_{i i}\right\}}-\frac{5}{2}\right) \frac{\tilde{T}_{i}}{T_{e}}\right] \\
& -\frac{4 \tau_{e i} i l q^{\prime}}{3 \sqrt{\pi} q} \frac{f_{t}}{\left\langle B^{2}\right\rangle}\left\langle\frac{I^{2}}{R^{2}}\right) \frac{T_{e}}{e} \frac{\mathrm{d}}{\mathrm{d} x}\left(x\left(\left[\frac{\tilde{p}_{e}}{p_{0 e}}-\frac{e(\Phi-\Psi)}{T_{e}}-\frac{\omega_{*_{e}}}{\omega}\left(1+\eta_{e}\right) \frac{e \Psi}{T_{e}}\right)\left\{u v_{e} D^{(1)}\right\}+\left(\frac{\tilde{T}_{e}}{T_{e}}-\frac{\omega_{x_{e}} \eta_{e}}{\omega} \frac{e \Psi}{T_{e}}\right)\left\{u v_{e} D^{(2)}\right\}\right]\right)
\end{aligned}
$$

leading to

$$
\begin{aligned}
& \frac{1}{n_{0} T_{e}} \frac{\mathrm{d}}{\mathrm{d} r}\left\langle Q_{r, e}^{B a n}\right\rangle=-1.70 f_{t} \frac{I^{2} m_{e} T_{e}}{e^{2}\left\langle B^{2}\right\rangle \tau_{e i}} \frac{\mathrm{d}^{2}}{\mathrm{~d} x^{2}}\left[\frac{\tilde{p}}{p_{0 e}}-0.40 \frac{\tilde{T}_{e}}{T_{e}}-1.17 \frac{\tilde{T}_{i}}{T_{e}}\right] \\
& -2.97 \frac{f_{t}}{\left\langle B^{2}\right\rangle}\left\langle\frac{I^{2}}{R^{2}}\right\rangle \frac{\mathrm{i} \ell q^{\prime} T_{e}}{e q} \frac{\mathrm{d}}{\mathrm{d} x}\left(x\left[\frac{\tilde{p}_{e}}{p_{0 e}}-\frac{e(\Phi-\Psi)}{T_{e}}-\frac{\omega_{* e}}{\omega}\left(1+1.25 \eta_{e}\right) \frac{e \Psi}{T_{e}}+0.25 \frac{\tilde{T}_{e}}{T_{e}}\right]\right)
\end{aligned} .
$$




\section{(ii) Parallel Electron Fluxes and Plasma Current}

The contribution to eqn. (59) from the parallel flux $\Gamma_{\| e}$ is

$$
\left\langle\frac{\mathrm{i} \ell I q^{\prime} x}{R^{2} B q} \int \mathrm{d}^{3} \boldsymbol{v} v_{\|} h_{1 e}\right\rangle=\left\langle\frac{\mathrm{i} \ell I^{\prime} x}{R^{2} B q} U_{\| e}\left(h_{1 e}\right)\right\rangle
$$

The part arising from the Pfirsch-Schlüter-like part of $h_{1 e}$ can be calculated directly, but for the banana contribution we take advantage of the selfadjointness of the collision operator to circumvent the fact that $h_{1 e}$ is not localised in pitch-angle, while still allowing us to use the model collision operator (Helander \& Sigmar 2002).

We first consider the parallel current, which is needed for Ampère's equation, but also provides an expression for $U_{\| e}$. We can readily calculate $j_{\|}^{P S}$, the current arising from the Pfirsch-Schlüter-like contributions to the electron and ion distribution functions given in eqns. (62) and (66), obtaining:

$$
\begin{aligned}
& j_{\|}^{P S}=-\widetilde{I}^{\prime}\left(\frac{1}{B}-\frac{B}{\left\langle B^{2}\right\rangle}\right)+\frac{4 \tau_{e i}}{3 \sqrt{\pi}} \frac{\mathrm{i} \ell q^{\prime} x p_{0 e} e}{m_{e} q} \\
& \times\left(\frac{1}{R^{2} B}-\frac{B}{\left\langle B^{2}\right\rangle}\left\langle\frac{1}{R^{2}}\right\rangle\right)\left[\left(\frac{\tilde{p}_{e}}{p_{0 e}}-\frac{e(\Phi-\Psi)}{T_{e}}-\frac{\omega_{* e}}{\omega}\left(1+\eta_{e}\right) \frac{e \Psi}{T_{e}}\right)\left\{\frac{D^{(1)}}{u}\right\}+\left(\frac{\tilde{T}_{e}}{T_{e}}-\frac{\omega_{* e} \eta_{e}}{\omega} \frac{e \Psi}{T_{e}}\right)\left\{\frac{D^{(2)}}{u}\right\}\right]
\end{aligned}
$$

which becomes 


$$
\begin{aligned}
j_{\|}^{P S}=-I \tilde{p}^{\prime}\left(\frac{1}{B}\right. & \left.-\frac{B}{\left\langle B^{2}\right\rangle}\right)-1.97 \tau_{e i} \frac{\mathrm{i} I \ell q^{\prime} x p_{0 e} e}{m_{e} q} \\
& \times\left(\frac{1}{R^{2} B}-\frac{B}{\left\langle B^{2}\right\rangle}\left\langle\frac{1}{R^{2}}\right\rangle\right)\left[\left(\frac{\tilde{p}_{e}}{p_{0 e}}-\frac{e(\Phi-\Psi)}{T_{e}}+\frac{0.34 \tilde{T}_{e}}{T_{e}}-\frac{\omega_{*_{e}}}{\omega}\left(1+1.34 \eta_{e}\right) \frac{e \Psi}{T_{e}}\right)\right]
\end{aligned}
$$

To calculate the banana regime neoclassical contribution, we write

$$
j_{\|}=j_{\| s}+\left(j_{\|}-j_{\| s}\right)=j_{\| s}-e \int \mathrm{d}^{3} v v_{\|}\left(h_{1 e}-h_{e s}\right)
$$

where

$$
j_{\| s}=n_{0} e U_{\| i}-e \int \mathrm{d}^{3} v v_{\|} h_{e s}
$$

so that

$$
\begin{aligned}
j_{\| s}=- & \frac{4 \tau_{e i}}{3 \sqrt{\pi}} n_{0} e \frac{\mathrm{i} \ell \mathrm{I} q^{\prime} x}{R^{2} B q} \frac{T_{e}}{m_{e}} \\
& \times\left[\left\{\frac{D^{(1)}(u)}{u}\right\}\left(\frac{\tilde{p}_{e}}{p_{0 e}}-\frac{e(\Phi-\Psi)}{T_{e}}-\frac{\omega_{*_{e}}}{\omega}\left(1+\eta_{e}\right) \frac{e \Psi}{T_{e}}\right)+\left\{\frac{D^{(2)}(u)}{u}\right\}\left(\frac{\tilde{T}_{e}}{T_{e}}-\frac{\omega_{*_{e}} \eta_{e}}{\omega} \frac{e \Psi}{T_{e}}\right)\right]
\end{aligned}
$$

which reduces to

$$
j_{\| s}=1.97 n_{0} e \tau_{e i} \frac{\mathrm{i} \ell I q^{\prime} x}{R^{2} B q} \frac{T_{e}}{m_{e}}\left[\frac{\tilde{p}_{e}}{p_{0 e}}-\frac{e(\Phi-\Psi)}{T_{e}}+0.34 \frac{\tilde{T}_{e}}{T_{e}}-\frac{\omega_{*_{e}}}{\omega}\left(1+1.34 \eta_{e}\right) \frac{e \Psi}{T_{e}}\right]
$$

Now, recalling eqn. (48), we can write

$$
j_{\|}-j_{\| s}=-e \int \mathrm{d}^{3} v v_{\|}\left(h_{1 e}-h_{e s}^{(1)}-h_{e s}^{(2)}\right)=-\frac{e R^{2} B}{S^{(1)}} \int \mathrm{d}^{3} v \frac{C_{e}\left(h_{s}^{(1)}\right)}{f_{0 e}}\left(h_{1 e}-h_{e s}^{(1)}-h_{e s}^{(2)}\right) .
$$


Using the self-adjointness property of $C_{e}\left(h_{s}^{(1)}\right)$, we obtain

$$
j_{\|}-j_{\| s}=-\frac{e R^{2} B}{S^{(1)}} \int \mathrm{d}^{3} v \frac{h_{s}^{(1)}}{f_{0 e}} C_{e}\left(h_{1 e}-h_{e s}^{(1)}-h_{e s}^{(2)}\right) .
$$

so that

$$
\begin{aligned}
\left(j_{\|}-j_{\| s}\right)= & -\frac{4 \tau_{e i}}{3 \sqrt{\pi}} e \int \mathrm{d}^{3} \boldsymbol{v} \frac{D^{(1)}(u)}{u} v_{\|} C_{e}\left(h_{1 e}-h_{e s}^{(1)}-h_{e s}^{(2)}\right) \\
& =\frac{4 \tau_{e i}}{3 \sqrt{\pi}} e \int \mathrm{d}^{3} \boldsymbol{v} \frac{D^{(1)}(u)}{u} v_{\|} v_{e}\left(h_{1 e}-h_{s}^{(1)}-h_{s}^{(2)}-\frac{m_{e} v_{\|}}{T_{e}} U_{\| i} f_{0 e}\right)
\end{aligned}
$$

where we have used the definitions (52) and the model electron-electron collision operator (50), since $\left(h_{1 e}-h_{e s}^{(1)}-h_{e s}^{(2)}\right)$ is now localised. Evaluating this expression using result (63)

$$
\begin{aligned}
&\left(j_{\|}-j_{\| s}\right)^{B a n}= \frac{4 \tau_{e i} n_{0}}{3 \sqrt{\pi}} \frac{f_{t} B I T_{e}}{\left\langle B^{2}\right\rangle}\left[\frac{\tilde{p}^{\prime}}{p_{0 e}}\left\{\frac{v_{e} D^{(1)}}{u}\right\}-\left\{\left(\frac{5}{2}-u^{2}\right) \frac{v_{e} D^{(1)}}{u}\right\} \frac{\tilde{T}_{e}^{\prime}}{T_{e}}-\left(\frac{5}{2}-\frac{\left\{u^{2} v_{i i}\right\}}{\left\{v_{i i}\right\}}\right)\left\{\frac{v_{e} D^{(1)}}{u}\right\} \frac{\tilde{T}_{i}^{\prime}}{T_{e}}\right] \\
&-\left(\frac{4 \tau_{e i}}{3 \sqrt{\pi}}\right)^{2} \frac{f_{t} B i I \ell q^{\prime} x n_{0}}{\left\langle B^{2}\right\rangle q}\left\langle\frac{1}{R^{2}}\right) \frac{e T_{e}}{m_{e}} \\
& \\
& \quad \times\left[\left(\frac{\tilde{p}_{e}}{p_{0 e}}-\frac{e(\Phi-\Psi)}{T_{e}}-\frac{\omega_{*_{e}}}{\omega}\left(1+\eta_{e}\right) \frac{e \Psi}{T_{e}}\right)\left\{\frac{v_{e} D^{(1) 2}}{u^{2}}\right\}+\left(\frac{\tilde{T}_{e}}{T_{e}}-\frac{\omega_{* e} \eta_{e}}{\omega} \frac{e \Psi}{T_{e}}\right)\left\{\frac{v_{e} D^{(1)} D^{(2)}}{u^{2}}\right\}\right]
\end{aligned}
$$

leading to

$$
\begin{aligned}
& \left(j_{\|}-j_{\| s}\right)^{B a n}=\frac{f_{t} B I p_{0 e}}{\left\langle B^{2}\right\rangle}\left[-1.67 \frac{\tilde{p}^{\prime}}{p_{0 e}}+1.19 \frac{\tilde{T}_{e}^{\prime}}{T_{e}}+1.95 \frac{\tilde{T}_{i}^{\prime}}{T_{e}}\right] \\
& -2.58 \frac{f_{t} B \text { i } I \ell q^{\prime} x p_{0 e} e \tau_{e i}}{\left\langle B^{2}\right\rangle q m_{e}}\left\langle\frac{1}{R^{2}}\right\rangle\left[\frac{\tilde{p}_{e}}{p_{0 e}}-\frac{e(\Phi-\Psi)}{T_{e}}-\frac{\omega_{*_{e}}}{\omega}\left(1+1.17 \eta_{e}\right) \frac{e \Psi}{T_{e}}+0.17 \frac{\tilde{T}_{e}}{T_{e}}\right]
\end{aligned}
$$

Combining expressions (85), (89) and (94), we obtain 


$$
\begin{aligned}
j_{\|}=-I \tilde{p}^{\prime}\left(\frac{1}{B}-\frac{B}{\left\langle B^{2}\right\rangle}\right)+1.97 \tau_{e i} \frac{\mathrm{i} I \ell q^{\prime} x p_{0 e} e}{m_{e} q} \frac{B}{\left\langle B^{2}\right\rangle}\left\langle\frac{1}{R^{2}}\right\rangle \\
\times\left[\left(1-1.31 f_{t}\right)\left(\frac{\tilde{p}_{e}}{p_{0 e}}-\frac{e(\Phi-\Psi)}{T_{e}}-\frac{\omega_{*_{e}}}{\omega}\left(1+\eta_{e}\right) \frac{e \Psi}{T_{e}}\right)+0.34\left(1-0.69 f_{t}\right)\left(\frac{\tilde{T}_{e}}{T_{e}}-\frac{\omega_{*_{e}} \eta_{e}}{\omega} \frac{e \Psi}{T_{e}}\right)\right] \\
\quad-\frac{f_{t} B I p_{0 e}}{\left\langle B^{2}\right\rangle}\left[1.67 \frac{\tilde{p}^{\prime}}{p_{0 e}}-1.19 \frac{\tilde{T}_{e}^{\prime}}{T_{e}}-1.95 \frac{\tilde{T}_{i}^{\prime}}{T_{e}}\right]
\end{aligned}
$$

The first term is the usual Pfirsch-Schlüter current while the second term represents the effects of the parallel electron pressure gradient, the parallel electric field and parallel thermal force, whereas the final term is the bootstrap current.

We can now calculate $U_{\| e}$ from

$$
U_{\| e}=U_{\| i}-\frac{j_{\|}}{n_{0} e}
$$

with $U_{\| i}$ given by eqn. (36). The contribution to the electron continuity equation from the divergence of the parallel flux (83) is then:

$$
\begin{aligned}
& n_{0}\left\langle\frac{\mathrm{i} \ell I q^{\prime} x}{R^{2} B q} U_{\| e}\right\rangle \\
= & \frac{\mathrm{i} I^{2} \ell q^{\prime} x}{e q} \tilde{p}^{\prime}\left(\left\langle\frac{1}{R^{2} B^{2}}\right\rangle-\frac{1}{\left\langle B^{2}\right\rangle}\left\langle\frac{1}{R^{2}}\right\rangle\right)+\frac{\mathrm{i} I^{2} \ell q^{\prime} x p_{0 \mathrm{e}} f_{t}}{e\left\langle B^{2}\right\rangle q}\left\langle\frac{1}{R^{2}}\right\rangle\left[1.67 \frac{\tilde{p}^{\prime}}{p_{0 e}}-1.19 \frac{\tilde{T}_{e}^{\prime}}{T_{e}}-3.12 \frac{\tilde{T}_{i}^{\prime}}{T_{e}}\right] \\
- & \frac{\mathrm{i} I^{2} \ell q^{\prime} x p_{0 e}}{e q}\left(\left\langle\frac{1}{R^{2} B^{2}}\right\rangle\left(\frac{p_{i}^{\prime}}{p_{0 e}}+\frac{e \Phi^{\prime}}{T_{e}}\right)-\frac{1.17}{\left\langle B^{2}\right\rangle}\left\langle\frac{1}{R^{2}}\right) \frac{\tilde{T}_{i}^{\prime}}{T_{e}}\right]-\frac{1.97 \tau_{e i}\left(I \ell q^{\prime} x\right)^{2} p_{0 e}}{m_{e}\left\langle B^{2}\right\rangle q^{2}}\left\langle\frac{1}{R^{2}}\right\rangle^{2} \\
\times & {\left[\left(1-1.31 f_{t}\right)\left(\frac{\tilde{p}_{e}}{p_{0 e}}-\frac{e(\Phi-\Psi)}{T_{e}}-\frac{\omega_{* e}}{\omega}\left(1+\eta_{e}\right) \frac{e \Psi}{T_{e}}\right)+0.34\left(1-0.69 f_{t}\right)\left(\frac{\tilde{T}_{e}}{T_{e}}-\frac{\omega_{* e} \eta_{e}}{\omega} \frac{e \Psi}{T_{e}}\right)\right] }
\end{aligned}
$$

The energy equation involves the parallel heat flux 


$$
Q_{\| e}=\int \mathrm{d}^{3} \boldsymbol{v} \frac{m_{e} v^{2}}{2} v_{\|} h_{1 e} \equiv q_{\| e}+\frac{5}{2} n_{0} T_{e} U_{\| e} ; q_{\| e}=\int \mathrm{d}^{3} v\left(\frac{m_{e} v^{2}}{2}-\frac{5}{2} T_{e}\right) v_{\|} h_{1 e}
$$

We can decompose $Q_{\| e}$ as

$$
Q_{\| e}=Q_{\| s e}+\left(q_{\| e}-q_{\| s e}\right)-\frac{5}{2 n_{0} e}\left(j_{\|}-j_{\| s}\right) T_{e} ; \quad Q_{\| s e}=\int \mathrm{d}^{3} v \frac{m_{e} v^{2}}{2} v_{\|} h_{\mathrm{se}}
$$

The Spitzer contribution is given as

$$
\begin{aligned}
Q_{\| s e}= & \frac{5}{2} n_{0} T_{e} U_{\| i}+\frac{4 \tau_{e i}}{3 \sqrt{\pi}} n_{0} T_{e} \frac{\mathrm{i} \ell I q^{\prime} x}{R^{2} B q} \frac{T_{e}}{m_{e}} \\
& \times\left[\left\{u D^{(1)}(u)\right\}\left(\frac{\tilde{p}_{e}}{p_{0 e}}-\frac{e(\Phi-\Psi)}{T_{e}}-\frac{\omega_{*_{e}}}{\omega}\left(1+\eta_{e}\right) \frac{e \Psi}{T_{e}}\right)+\left\{u D^{(2)}(u)\right\}\left(\frac{\tilde{T}_{e}}{T_{e}}-\frac{\omega_{*_{e}} \eta_{e}}{\omega} \frac{e \Psi}{T_{e}}\right)\right],(100)
\end{aligned}
$$

resulting in

$$
\begin{aligned}
Q_{\| s e}=- & \frac{5}{2} n_{0} T_{e} \frac{I T_{i}}{e B}\left[\left(\frac{p_{i}^{\prime}}{p_{0 e}}+\frac{e \Phi^{\prime}}{T_{e}}\right)-\frac{1.17 B^{2}}{\left\langle B^{2}\right\rangle}\left(1-f_{t}\right) \frac{\tilde{T}_{i}^{\prime}}{T_{e}}\right] \\
& -6.26 n_{0} T_{e} \frac{\mathrm{i} \ell I q^{\prime} x \tau_{e i}}{R^{2} B q} \frac{T_{e}}{m_{e}}\left(\frac{\tilde{p}_{e}}{p_{0 e}}-\frac{e(\Phi-\Psi)}{T_{e}}+0.58 \frac{\tilde{T}_{e}}{T_{e}}-\frac{\omega_{*_{e}}}{\omega}\left(1+1.58 \eta_{e}\right) \frac{e \Psi}{T_{e}}\right)
\end{aligned}
$$

Introducing the Pfirsch-Schlüter part of $h_{1 e}$, eqn. (62), we can calculate the corresponding Pfirsch-Schlüter contribution to $Q_{\| e}$ directly, obtaining :

$$
\begin{aligned}
\frac{Q_{\| e}^{P S}}{p_{0 e}}=\frac{5}{2} \frac{I T_{e}}{e}\left(\frac{1}{B}-\frac{B}{\left\langle B^{2}\right\rangle}\right)\left(\frac{\tilde{p}^{\prime}}{p_{0 e}}+\right. & \left.\frac{\tilde{T}_{e}^{\prime}}{T_{e}}\right)+6.26 n_{0} T \tau_{e i} \frac{\mathrm{i} I \ell q^{\prime} x T_{e}}{m_{e} q}\left(\frac{1}{R^{2} B}-\frac{B}{\left\langle B^{2}\right\rangle}\left\langle\frac{1}{R^{2}}\right\rangle\right) \\
& \times\left[\left(\frac{\tilde{p}_{e}}{p_{0 e}}-\frac{e(\Phi-\Psi)}{T_{e}}+\frac{0.58 \tilde{T}_{e}}{T_{e}}-\frac{\omega_{*_{e}}}{\omega}\left(1+1.58 \eta_{e}\right) \frac{e \Psi}{T_{e}}\right)\right]
\end{aligned}
$$


We formulate the banana neoclassical contribution for $q_{\| e}$ by analogy to the neoclassical current, $j_{\| e}$, as in eqn. (92), so that it can also be evaluated using the localised distribution function (63):

$$
\left(q_{\| e}-q_{\| s e}\right)=-\frac{4 \tau_{e i}}{3 \sqrt{\pi}} T_{e} \int \mathrm{d}^{3} \boldsymbol{v} \frac{D^{(2)}(u)}{u} v_{\|} v_{e}\left(h_{1 e}-h_{s}^{(1)}-h_{s}^{(2)}-\frac{m_{e} v_{\|}}{T_{e}} U_{\| i} f_{0 e}\right)
$$

Using expression (63), we obtain the banana contribution to $q_{\| e}$ :

$$
\begin{aligned}
& \left(q_{\| e}-q_{\| s e}\right)^{B a n}=\frac{f_{t} B I T_{e} p_{0 e}}{e\left\langle B^{2}\right\rangle}\left[0.04 \frac{\tilde{p}^{\prime}}{p_{0 e}}+0.64 \frac{\tilde{T}_{e}^{\prime}}{T_{e}}-0.05 \frac{\tilde{T}_{i}^{\prime}}{T_{e}}\right] \\
& +0.46 \frac{f_{t} \mathrm{i} I \ell q^{\prime} x T_{e} p_{0 e} \tau_{e i}}{m_{e} q} \frac{B}{\left\langle B^{2}\right\rangle}\left\langle\frac{1}{R^{2}}\right\rangle\left[\frac{\tilde{p}_{e}}{p_{0 e}}-\frac{e(\Phi-\Psi)}{T_{e}}-\frac{\omega_{*_{e}}}{\omega}\left(1+1.94 \eta_{e}\right) \frac{e \Psi}{T_{e}}+0.94 \frac{\tilde{T}_{e}}{T_{e}}\right]
\end{aligned}
$$

Inserting the results (94), (101), (102) and (104) into eqn. (99), we finally obtain

$$
\begin{aligned}
& \frac{1}{n_{0} T_{e}}\left\langle\frac{\mathrm{i} \ell I q^{\prime} x}{R^{2} B q} Q_{\| l e}\right\rangle=\frac{5}{2} \frac{\mathrm{i} \ell I^{2} q^{\prime} x T_{e}}{e q}\left(\left\langle\frac{1}{R^{2} B^{2}}\right\rangle-\frac{1}{\left\langle B^{2}\right\rangle}\left\langle\frac{1}{R^{2}}\right\rangle\right)\left(\frac{\tilde{p}^{\prime}}{p_{0 e}}+\frac{\tilde{T}_{e}^{\prime}}{T_{e}}\right) \\
&+\frac{f_{t}}{\left\langle B^{2}\right\rangle}\left\langle\frac{1}{R^{2}}\right\rangle \frac{\mathrm{i} \ell I^{2} q^{\prime} x T_{e}}{e q}\left[4.18 \frac{\tilde{p}^{\prime}}{p_{0 e}}-2.36 \frac{\tilde{T}_{e}^{\prime}}{T_{e}}-7.86 \frac{\tilde{T}_{i}^{\prime}}{T_{e}}\right] \\
&-\frac{5}{2} \frac{\mathrm{i} \ell I^{2} q^{\prime} x T_{i}}{e q}\left[\left\langle\frac{1}{R^{2} B^{2}}\right\rangle\left(\frac{p_{i}^{\prime}}{p_{0 e}}+\frac{e \Phi^{\prime}}{T_{e}}\right)-\frac{1.17}{\left\langle B^{2}\right\rangle}\left\langle\frac{1}{R^{2}}\right) \frac{\tilde{T}_{i}^{\prime}}{T_{e}}\right]+6.26 \frac{\left(\ell I q^{\prime} x\right)^{2}}{\left\langle B^{2}\right\rangle q^{2}}\left\langle\frac{1}{R^{2}}\right\rangle^{2} \frac{T_{e} \tau_{e i}}{m_{e}} \\
& \times\left[\left(1-1.11 f_{t}\right)\left(\frac{\tilde{p}_{e}}{p_{0 e}}-\frac{e(\Phi-\Psi)}{T_{e}}-\frac{\omega_{*_{e}}}{\omega} \frac{e \Psi}{T_{e}}\right)+0.58\left(1-0.31 f_{t}\right)\left(\frac{\tilde{T}_{e}}{T_{e}}-\frac{\eta_{e} \omega_{*_{e}}}{\omega} \frac{e \Psi}{T_{e}}\right)\right] .
\end{aligned}
$$




\section{(iii) Azimuthal Electron Fluxes}

Finally, we require the contributions from the azimuthal drift terms that appear in eqns. (59) and (60).

$$
\begin{aligned}
\left\langle\int \mathrm{d}^{3} \boldsymbol{v} \boldsymbol{v}_{d e} \cdot \nabla h_{0 e}\right\rangle & =-\mathrm{i}\left\langle\iint \mathrm{d}^{3} \boldsymbol{v} \boldsymbol{v}_{d e} \cdot(m \nabla \theta-\ell \nabla \varphi) h_{0 e}\right\rangle \\
= & -\mathrm{i}\left\langle\frac{\boldsymbol{n}}{\Omega_{e}} \times(\nabla \ln B+\boldsymbol{\kappa}) \cdot(m \nabla \theta-\ell \nabla \varphi)\right\rangle \frac{T_{e}}{m_{e}}\left(\frac{\hat{n}_{e}}{n_{0}}+\frac{\hat{T}_{e}}{T_{e}}\right) n_{0} \\
= & -\mathrm{i}\left\langle\frac{\boldsymbol{n}}{\Omega_{e}} \times \frac{\nabla\left(\mu_{0} p_{0}+B^{2}\right)}{B^{2}} \cdot(m \nabla \theta-\ell \nabla \varphi)\right) \frac{T_{e}}{m_{e}}\left(\frac{\hat{n}_{e}}{n_{0}}+\frac{\hat{T}_{e}}{T_{e}}\right) n_{0} .
\end{aligned}
$$

This can be expressed as

$$
\begin{array}{r}
\left\langle\int \mathrm{d}^{3} \boldsymbol{v} \boldsymbol{v}_{d e} \cdot \nabla h_{0 e}\right\rangle \\
=\mathrm{i} \ell \frac{T_{e}}{e}\left\langle\left(\frac{1}{B^{2}} \frac{\partial}{\partial \chi}\left(\mu_{0} p_{0}+B^{2}\right)+\left(\frac{1}{R B^{2}}\right)^{2} \nabla \chi \cdot \nabla \theta \frac{\partial B^{2}}{\partial \theta}\right)\right\rangle \\
\times\left(\frac{\tilde{p}_{e}}{p_{0 e}}-\frac{e(\Phi-\Psi)}{T_{e}}-\frac{\omega_{* e}}{\omega}\left(1+\eta_{e}\right) \frac{e \Psi}{T_{e}}\right) n_{0}
\end{array}
$$

Similarly, we also obtain

$$
\begin{aligned}
& \left\langle\int \mathrm{d}^{3} \boldsymbol{v} \frac{m_{e} v^{2}}{2} v_{d e} \cdot \nabla h_{0 e}\right\rangle \\
& =\frac{5}{2} i \ell \frac{T_{e}}{e}\left\langle\left(\frac{1}{B^{2}} \frac{\partial}{\partial \chi}\left(\mu_{0} p_{0}+B^{2}\right)+\left(\frac{1}{R B^{2}}\right)^{2} \nabla \chi \cdot \nabla \theta \frac{\partial B^{2}}{\partial \theta}\right)\right) \\
& \quad \times\left(\frac{\tilde{p}_{e}}{p_{0 e}}+\frac{\tilde{T}_{e}}{T_{e}}-\frac{e(\Phi-\Psi)}{T_{e}}-\frac{\omega_{*_{e}}}{\omega}\left(1+2 \eta_{e}\right) \frac{e \Psi}{T_{e}}\right) n_{0} T_{e}
\end{aligned}
$$




\section{(b) The Perturbed Ion Temperature}

In the case of the ions there is no need to obtain the ion density equation for $\hat{n}_{i}$ as it is determined by quasi-neutrality and given in eqn. (13). To obtain the equation for $\hat{T}_{i}$ we apply the operator $\int(\ldots)\left(m_{i} v^{2} / 2\right) \mathrm{d}^{3} v$ to eqn. (37).

$$
\begin{aligned}
&\left\langle\int \mathrm{d}^{3} \boldsymbol{v}\right.\left.\frac{v_{\|} I}{R^{2} B q} \frac{\partial}{\partial \theta}\left(\frac{I v_{\|}}{\Omega_{e}}\right) \frac{m_{i} v^{2}}{2} \frac{\partial h_{2 i}}{\partial x}\right\rangle+\left\langle\int \mathrm{d}^{3} \boldsymbol{v} \frac{m_{i} v^{2}}{2} v_{d \theta i} \cdot \nabla h_{0 i}\right\rangle \\
&-\left\langle\int \mathrm{d}^{3} \boldsymbol{v} \frac{m_{i} v^{2}}{2} \overline{L_{i} C_{i i}\left(g_{i} L_{i}\right)}\right\rangle=\frac{3}{2} \mathrm{i} \omega\left(\frac{\tilde{p}_{i}}{p_{0 i}}+\frac{e \Phi}{T_{i}}-\frac{e \Psi}{T_{i}}\left(1+\frac{T_{i}}{T_{e}} \frac{\omega_{*_{e}}}{\omega}\left(1+\eta_{i}\right)\right)\right) \\
& \quad-\mathrm{i} n_{0}\left\langle\frac{3}{2} \frac{e}{T_{i}}\left(\omega+\frac{T_{i}}{T_{e}} \omega_{*_{e}}\left(1+\eta_{i}\right)\right)(\Phi-\Psi)+\frac{5}{2} \frac{\delta B_{\|}}{B}\left(\omega+\frac{T_{i}}{T_{e}} \omega_{*_{e}}\left(1+2 \eta_{i}\right)\right)\right\rangle
\end{aligned}
$$

Integrating the first term on the left hand side by parts and substituting for $h_{2 i}$ from eqn. (25), it can be written as the divergence of the neoclassical ion heat flux

$$
\frac{\mathrm{d}}{\mathrm{d} r}\left\langle Q_{i}^{N C}\right\rangle \cong \frac{\mathrm{d}}{\mathrm{d} x}\left\langle\int \mathrm{d}^{3} \boldsymbol{v} \frac{m_{i} v^{2}}{2} \frac{I v_{\|}}{\Omega_{i}} C_{i i}\left(h_{1 i}\right)\right\rangle .
$$

The second term is the ion azimuthal flux and the third is the ion classical heat flux:

$$
\frac{\mathrm{d}}{\mathrm{d} r}\left\langle Q_{r i}^{C}\right\rangle=\left\langle\int \mathrm{d}^{3} \boldsymbol{v} \frac{m_{i} v^{2}}{2} \overline{L_{i} C_{i i}\left(g_{0 i} L_{i}\right)}\right\rangle .
$$

This can be evaluated in a similar manner to the electron case, resulting in 


$$
\frac{\mathrm{d}}{\mathrm{d} x}\left\langle Q_{r i}^{C}\right\rangle=-2 \frac{m_{i} T_{i}}{e^{2} \tau_{i}}\left\langle\frac{|\nabla \chi|^{2}}{B^{2}}\right\rangle n_{0} \frac{\mathrm{d}^{2}}{\mathrm{~d} x^{2}} \tilde{T}_{i},
$$

where $\tau_{i}=(3 \sqrt{\pi} / 4) 4 \pi \varepsilon_{0}^{2} m_{i}^{1 / 2} T_{i}^{3 / 2} / n_{0} e^{4} \ln \Lambda$.

The calculation of the Pfirsch-Schlüter heat flux proceeds similarly to the electron one and yields

$$
\frac{\mathrm{d}}{\mathrm{d} r}\left\langle Q_{r i}^{P S}\right\rangle=-\frac{2 m_{i} T_{i}}{e^{2} \tau_{i}} I^{2}\left(\left\langle\frac{1}{B^{2}}\right\rangle-\frac{1}{\left\langle B^{2}\right\rangle}\right) n_{0} \frac{\mathrm{d}^{2}}{\mathrm{~d} x^{2}} \widetilde{T}_{i},
$$

while the banana regime contribution is

$$
\frac{\mathrm{d}}{\mathrm{d} r}\left\langle Q_{r i}^{\text {Ban }}\right\rangle=-\frac{m_{i} T_{i} I^{2} f_{t}}{e^{2}\left\langle B^{2}\right\rangle}\left(\left\{u^{4} v_{i i}\right\}-\frac{\left\{u^{2} v_{i i}\right\}^{2}}{\left\{v_{i i}\right\}}\right) n_{0} \frac{\mathrm{d}^{2}}{\mathrm{~d} x^{2}} \widetilde{T}_{i}=-0.92 \frac{m_{i} T_{i} I^{2} f_{t}}{e^{2}\left\langle B^{2}\right\rangle \tau_{i}} n_{0} \frac{\mathrm{d}^{2}}{\mathrm{~d} x^{2}} \widetilde{T}_{i} .
$$

Finally, the azimuthal drift contribution is

$$
\begin{array}{r}
\left\langle\int \mathrm{d}^{3} \boldsymbol{v} \frac{m_{i} v^{2}}{2} \boldsymbol{v}_{d i} \cdot \nabla h_{0 i}\right\rangle=-\frac{5}{2} i \ell \frac{T_{i}}{e}\left\langle\left(\frac{1}{B^{2}} \frac{\partial}{\partial \chi}\left(\mu_{0} p_{0}+B^{2}\right)+\left(\frac{1}{R B^{2}}\right)^{2} \nabla \chi \cdot \nabla \theta \frac{\partial B^{2}}{\partial \theta}\right)\right\rangle \\
\times\left(\frac{\tilde{p}_{i}}{p_{0 i}}+\frac{\tilde{T}_{i}}{T_{i}}+\frac{e(\Phi-\Psi)}{T_{i}}-\frac{\omega_{*_{e}}}{\omega}\left(1+2 \eta_{i}\right) \frac{e \Psi}{T_{e}}\right) n_{0} T_{i}
\end{array}
$$

\section{(c) Summary}

Here we collect together the above results to obtain the final form of the equations determining the electron density and temperature perturbations in 
terms of the perturbed fields $\Phi$ and $\Psi$. The first, eqn. (59) can be written in the form

$$
\begin{aligned}
\frac{\mathrm{d}}{\mathrm{d} r}\left(\left\langle\Gamma_{r, e}^{C}\right\rangle+\left\langle\Gamma_{r, e}^{P S}\right\rangle+\langle\right. & \left.\left.\Gamma_{r, e}^{B a n}\right\rangle\right)+\mathrm{i} k_{\| 0}\left\langle\Gamma_{\|, e}\right\rangle+\mathrm{i} k_{\theta}\left\langle\Gamma_{e}^{A z}\right\rangle \\
& =\mathrm{i} \omega\left[\tilde{n}-\frac{\omega_{* e}}{\omega} \frac{e \Phi}{T_{e}} n_{0}+\left\langle\frac{\mu_{0} \tilde{p}}{B^{2}}\right\rangle\left(1-\frac{\omega_{* e}}{\omega}\left(1+\eta_{e}\right)\right) n_{0}\right]
\end{aligned}
$$

where $k_{\| 0}=\ell I q^{\prime} x\left\langle 1 / R^{2} B\right\rangle / q, k_{\theta}=\ell q\langle|\nabla \theta|\rangle$ and we have substituted eqn. (58) for $\delta B_{\|}$.

From eqn. (70) we have

$$
\frac{\mathrm{d}}{\mathrm{d} r}\left\langle\Gamma_{r, e}^{C}\right\rangle=-\frac{m_{e} T_{e}}{e^{2} \tau_{e i}} \frac{\mathrm{d}^{2}}{\mathrm{~d} x^{2}}\left\langle\frac{|\nabla \chi|^{2}}{B^{2}}\right\rangle\left(\frac{\tilde{p}}{p_{0 e}}-\frac{3}{2} \frac{\tilde{T}_{e}}{T_{e}}\right) n_{0},
$$

From eqn. (76)

$$
\begin{aligned}
& \frac{1}{n_{0}} \frac{\mathrm{d}}{\mathrm{d} r}\left\langle\Gamma_{r, e}^{P S}\right\rangle=-\frac{m_{e} T_{e}}{e^{2} \tau_{e i}} I^{2}\left(\left\langle\frac{1}{B^{2}}\right\rangle-\frac{1}{\left\langle B^{2}\right\rangle}\right) \frac{\mathrm{d}^{2}}{\mathrm{~d} x^{2}}\left(\frac{\tilde{p}}{p_{0 e}}-\frac{3}{2} \frac{\tilde{T}_{e}}{T_{e}}\right) \\
& -\frac{\mathrm{i} I^{2} \ell q^{\prime} T_{e}}{e q}\left(\left\langle\frac{1}{R^{2} B^{2}}\right\rangle-\frac{1}{\left\langle B^{2}\right\rangle}\left\langle\frac{1}{R^{2}}\right\rangle\right) \frac{\mathrm{d}}{\mathrm{d} x}\left[x\left(\frac{\tilde{p}_{e}}{p_{0 e}}-\frac{e \Phi}{T_{e}}+\left(1-\frac{\omega_{*_{e}}}{\omega}\left(1+\eta_{e}\right)\right) \frac{e \Psi}{T_{e}}\right)\right]
\end{aligned}
$$

From eqn. (80)

$$
\begin{aligned}
& \frac{1}{n_{0}} \frac{\mathrm{d}}{\mathrm{dr}}\left\langle\Gamma_{r, e}^{B a n}\right\rangle=-1.53 f_{t} \frac{I^{2} m_{e} T_{e}}{e^{2}\left\langle B^{2}\right\rangle \tau_{e i}} \frac{\mathrm{d}^{2}}{\mathrm{~d} x^{2}}\left[\frac{\tilde{p}}{p_{0 e}}+1.39 \frac{\tilde{T}_{e}}{T_{e}}-1.17 \frac{\tilde{T}_{i}}{T_{e}}\right] \\
& -1.67 \frac{f_{t}}{\left\langle B^{2}\right\rangle}\left\langle\frac{I^{2}}{R^{2}}\right\rangle \frac{\mathrm{i} \ell q^{\prime} T_{e}}{e q} \frac{\mathrm{d}}{\mathrm{d} x}\left(x\left[\frac{\tilde{p}_{e}}{p_{0 e}}-\frac{e(\Phi-\Psi)}{T_{e}}-\frac{\omega_{*_{e}}}{\omega}\left(1+1.02 \eta_{e}\right) \frac{e \Psi}{T_{e}}+0.02 \frac{\tilde{T}_{e}}{T_{e}}\right]\right)
\end{aligned}
$$

From eqn. (97), we have defined 


$$
\begin{aligned}
& \left\langle\frac{1}{R^{2} B}\right\rangle\left\langle\Gamma_{\|, e}\right\rangle= \\
& \frac{I}{e} \tilde{p}^{\prime}\left(\left\langle\frac{1}{R^{2} B^{2}}\right\rangle-\frac{1}{\left\langle B^{2}\right\rangle}\left\langle\frac{1}{R^{2}}\right\rangle\right)+\frac{I p_{0 e} f_{t}}{e\left\langle B^{2}\right\rangle}\left\langle\frac{1}{R^{2}}\right\rangle\left[1.67 \frac{\tilde{p}^{\prime}}{p_{0 e}}-1.19 \frac{\tilde{T}_{e}^{\prime}}{T_{e}}-3.12 \frac{\tilde{T}_{i}^{\prime}}{T_{e}}\right] \\
& -\frac{I p_{0 e}}{e}\left[\left\langle\frac{1}{R^{2} B^{2}}\right\rangle\left(\frac{p_{i}^{\prime}}{p_{0 e}}+\frac{e \Phi^{\prime}}{T_{e}}\right)-\frac{1.17}{\left\langle B^{2}\right\rangle}\left\langle\frac{1}{R^{2}}\right\rangle \frac{\tilde{T}_{i}^{\prime}}{T_{e}}\right]-\mathrm{i} \frac{1.97 \tau_{e i}\left(I \ell q^{\prime} x\right) p_{0 e}}{m_{e}\left\langle B^{2}\right\rangle q}\left\langle\frac{1}{R^{2}}\right\rangle^{2} \\
& \quad \times\left[\left(1-1.31 f_{t}\right)\left(\frac{\tilde{p}_{e}}{p_{0 e}}-\frac{e(\Phi-\Psi)}{T_{e}}-\frac{\omega_{* e}}{\omega}\left(1+\eta_{e}\right) \frac{e \Psi}{T_{e}}\right)+0.34\left(\frac{\tilde{T}_{e}}{T_{e}}-\frac{\omega_{* e} \eta_{e}}{\omega} \frac{e \Psi}{T_{e}}\right)\left(1-0.69 f_{t}\right)\right]
\end{aligned}
$$

while from eqn. (107) we have defined

$$
\begin{array}{r}
\left\langle\Gamma_{e}^{A z}\right\rangle=\frac{T_{e}}{e q\langle|\nabla \theta|\rangle}\left\langle\left(\frac{1}{B^{2}} \frac{\partial}{\partial \chi}\left(\mu_{0} p_{0}+B^{2}\right)+\left(\frac{1}{R B^{2}}\right)^{2} \nabla \chi \cdot \nabla \theta \frac{\partial B^{2}}{\partial \theta}\right)\right\rangle \\
\times\left(\frac{\tilde{p}_{e}}{p_{0 e}}-\frac{e(\Phi-\Psi)}{T_{e}}-\frac{\omega_{*_{e}}}{\omega}\left(1+\eta_{e}\right) \frac{e \Psi}{T_{e}}\right) n_{0}
\end{array}
$$

Similarly, for eqn. (60) we obtain

$$
\begin{aligned}
& \frac{\mathrm{d}}{\mathrm{d} r}\left(\left\langle Q_{r, e}^{C}\right\rangle+\left\langle Q_{r, e}^{P S}\right\rangle+\left\langle Q_{r, e}^{B a n}\right\rangle\right)+\mathrm{i} k_{\| 0}\left\langle Q_{\| e}\right\rangle+\mathrm{i} k_{\theta}\left\langle Q_{e}^{A z}\right\rangle \\
& \quad=\frac{3}{2} \mathrm{i} \omega\left[\tilde{p}_{e}-\frac{\omega_{*_{e}}\left(1+\eta_{e}\right)}{\omega} e \Phi_{0}+\frac{5}{3}\left\langle\frac{\mu_{0} \tilde{p}}{B^{2}}\right\rangle\left(1-\frac{\omega_{*_{e}}}{\omega}\left(1+2 \eta_{e}\right)\right) p_{0 e}\right]
\end{aligned}
$$

where, from eqn. (72),

$$
\frac{\mathrm{d}}{\mathrm{d} r}\left\langle Q_{r, e}^{C}\right\rangle=-\frac{m_{e} T_{e}}{e^{2} \tau_{e i}}\left\langle\frac{|\nabla \chi|^{2}}{B^{2}}\right\rangle \frac{\mathrm{d}^{2}}{\mathrm{~d} x^{2}}\left(\frac{\tilde{p}}{p_{0 e}}+0.91 \frac{\tilde{T}_{e}}{T_{e}}\right) n_{0} T_{e}
$$

from eqn. (77), 


$$
\begin{aligned}
& \frac{1}{\mathrm{n}_{0} T_{e}} \frac{\mathrm{d}}{\mathrm{d} r}\left\langle Q_{r, e}^{P S}\right\rangle=-\frac{m_{e} T_{e} I^{2}}{e^{2} \tau_{e i}}\left(\left\langle\frac{1}{B^{2}}\right\rangle-\frac{1}{\left\langle B^{2}\right\rangle}\right) \frac{\mathrm{d}^{2}}{\mathrm{~d} x^{2}}\left(\frac{\tilde{p}}{p_{0 e}}+0.91 \frac{\tilde{T}_{e}}{T_{e}}\right) \\
& -\frac{\mathrm{i} I^{2} \ell q^{\prime} T_{e}}{e q}\left(\left\langle\frac{1}{R^{2} B^{2}}\right\rangle-\frac{1}{\left\langle B^{2}\right\rangle}\left\langle\frac{1}{R^{2}}\right\rangle\right) \frac{\mathrm{d}}{\mathrm{d} x}\left[\frac{5}{2} x\left(\frac{\tilde{p}_{e}}{p_{0 e}}-\frac{e \Phi}{T_{e}}+\frac{\tilde{T}_{e}}{T_{e}}+\frac{e \Psi}{T_{e}}\left(1-\frac{\omega_{*_{e}}}{\omega}\left(1+2 \eta_{e}\right)\right)\right)\right]
\end{aligned}
$$

and from eqn. (82),

$$
\begin{aligned}
& \frac{1}{n_{0} T_{e}} \frac{\mathrm{d}}{\mathrm{d} r}\left\langle Q_{r, e}^{B a n}\right\rangle=-1.70 f_{t} \frac{I^{2} m_{e} T_{e}}{e^{2}\left\langle B^{2}\right\rangle \tau_{e i}} \frac{\mathrm{d}^{2}}{\mathrm{~d} x^{2}}\left[\frac{\tilde{p}}{p_{0 e}}-0.40 \frac{\tilde{T}_{e}}{T_{e}}-1.17 \frac{\tilde{T}_{i}}{T_{e}}\right] \\
& -2.97 \frac{f_{t}}{\left\langle B^{2}\right\rangle}\left\langle\frac{I^{2}}{R^{2}}\right\rangle \frac{\mathrm{i} \ell q^{\prime} T_{e}}{e q} \frac{\mathrm{d}}{\mathrm{d} x}\left(x\left[\frac{\tilde{p}_{e}}{p_{0 e}}-\frac{e(\Phi-\Psi)}{T_{e}}-\frac{\omega_{*_{e}}}{\omega}\left(1+1.25 \eta_{e}\right) \frac{e \Psi}{T_{e}}+0.25 \frac{\tilde{T}_{e}}{T_{e}}\right]\right),
\end{aligned}
$$

while, from eqn. (105),

$$
\begin{aligned}
\frac{1}{n_{0} T_{e}}\left\langle\frac{1}{R^{2} B}\right\rangle\left\langle Q_{\| e}\right\rangle & =\frac{5}{2} \frac{I T_{e}}{e}\left(\left\langle\frac{1}{R^{2} B^{2}}\right\rangle-\frac{1}{\left\langle B^{2}\right\rangle}\left\langle\frac{1}{R^{2}}\right\rangle\right)\left(\frac{\tilde{p}^{\prime}}{p_{0 e}}+\frac{\tilde{T}_{e}^{\prime}}{T_{e}}\right) \\
+ & \frac{f_{t}\left\langle R^{2} B\right\rangle}{\left\langle B^{2}\right\rangle}\left\langle\frac{1}{R^{2}}\right\rangle \frac{I T_{e}}{e}\left[4.18 \frac{\tilde{p}^{\prime}}{p_{0 e}}-2.36 \frac{\tilde{T}_{e}^{\prime}}{T_{e}}-7.86 \frac{\tilde{T}_{i}^{\prime}}{T_{e}}\right] \\
- & \frac{5}{2} \frac{\mathrm{i} \ell I^{2} q^{\prime} x T_{i}}{e q}\left[\left\langle\frac{1}{R^{2} B^{2}}\right\rangle\left(\frac{p_{i}^{\prime}}{p_{0 e}}+\frac{e \Phi^{\prime}}{T_{e}}\right)-\frac{1.17}{\left\langle B^{2}\right\rangle}\left\langle\frac{1}{R^{2}}\right) \frac{\tilde{T}_{i}^{\prime}}{T_{e}}\right]-6.26 \frac{\mathrm{i} \ell I q^{\prime} x}{\left\langle B^{2}\right\rangle q}\left\langle\frac{1}{R^{2}}\right\rangle^{2} \frac{T_{e} \tau_{e i}}{m_{e}} \\
& \times\left[\left(1-1.11 f_{t}\right)\left(\frac{\tilde{p}_{e}}{p_{0 e}}-\frac{e(\Phi-\Psi)}{T_{e}}-\frac{\omega_{*_{e}}}{\omega} \frac{e \Psi}{T_{e}}\right)+0.58\left(1-0.31 f_{t}\right)\left(\frac{\tilde{T}_{e}}{T_{e}}-\frac{\eta_{e} \omega_{*_{e}}}{\omega} \frac{e \Psi}{T_{e}}\right)\right]
\end{aligned}
$$

and, from eqn. (108), 


$$
\begin{aligned}
\left\langle Q_{e}^{A z}\right\rangle=\frac{5}{2} \frac{T_{e}}{q e(|\nabla \theta|\rangle}\left\langle\left(\frac{1}{B^{2}} \frac{\partial}{\partial \chi}\left(\mu_{0} p_{0}+B^{2}\right)+\left(\frac{1}{R B^{2}}\right)^{2} \nabla \chi \cdot \nabla \theta \frac{\partial B^{2}}{\partial \theta}\right)\right\rangle \\
\times\left(\frac{\tilde{p}_{e}}{p_{0 e}}+\frac{\tilde{T}_{e}}{T_{e}}-\frac{e(\Phi-\Psi)}{T_{e}}-\frac{\omega_{\psi_{e}}}{\omega}\left(1+2 \eta_{e}\right) \frac{e \Psi}{T_{e}}\right) n_{0} T_{e}
\end{aligned}
$$

Summarising for the ions, we can rewrite eqn. (109) as:

$$
\begin{aligned}
& \frac{\mathrm{d}}{\mathrm{d} r}\left(\left\langle Q_{r, i}^{C}\right\rangle+\left\langle Q_{r, i}^{P S}\right\rangle+\left\langle Q_{r, i}^{B a n}\right\rangle\right)+\mathrm{i} k_{\theta}\left\langle Q_{i}^{A z}\right\rangle \\
& \quad=\frac{3}{2} \mathrm{i} \omega\left[\tilde{p}_{i}-\frac{\omega_{* e}\left(1+\eta_{i}\right)}{\omega} \frac{T_{i}}{T_{e}} e \Phi n_{0}+\frac{5}{3} p_{0 i}\left\langle\frac{\mu_{0} \tilde{p}}{B^{2}}\right\rangle\left(1+\frac{T_{i}}{T_{e}} \frac{\omega_{*_{e}}}{\omega}\left(1+2 \eta_{i}\right)\right)\right],
\end{aligned}
$$

where, from eqn. (112),

$$
\frac{\mathrm{d}}{\mathrm{d} x}\left\langle Q_{r i}^{C}\right\rangle=-2 \frac{m_{i} T_{i}}{e^{2} \tau_{i}}\left\langle\frac{|\nabla \chi|^{2}}{B^{2}}\right\rangle n_{0} \frac{\mathrm{d}^{2}}{\mathrm{~d} x^{2}} \tilde{T}_{i},
$$

from eqn. (113)

$$
\frac{\mathrm{d}}{\mathrm{d} r}\left\langle Q_{r i}^{P S}\right\rangle=-\frac{2 m_{i} T_{i}}{e^{2} \tau_{i}} I^{2}\left(\left\langle\frac{1}{B^{2}}\right\rangle-\frac{1}{\left\langle B^{2}\right\rangle}\right) n_{0} \frac{\mathrm{d}^{2}}{\mathrm{~d} x^{2}} \tilde{T}_{i},
$$

from eqn. (114)

$$
\frac{\mathrm{d}}{\mathrm{d} r}\left\langle Q_{r i}^{\text {Ban }}\right\rangle=-0.92 \frac{m_{i} T_{i} I^{2} f_{t}}{e^{2}\left\langle B^{2}\right\rangle \tau_{i}} n_{0} \frac{\mathrm{d}^{2}}{\mathrm{~d} x^{2}} \tilde{T}_{i}
$$

and, finally, from eqn. (115): 


$$
\begin{aligned}
&\left\langle Q_{i}^{A z}\right\rangle=-\frac{5}{2} \frac{T_{i}}{e q\langle|\nabla \theta|\rangle}\left\langle\left(\frac{1}{B^{2}} \frac{\partial}{\partial \chi}\left(\mu_{0} p_{0}+B^{2}\right)+\left(\frac{1}{R B^{2}}\right)^{2} \nabla \chi \cdot \nabla \theta \frac{\partial B^{2}}{\partial \theta}\right)\right\rangle \\
& \times\left(\frac{\tilde{p}_{i}}{p_{0 i}}+\frac{\tilde{T}_{i}}{T_{i}}+\frac{e(\Phi-\Psi)}{T_{i}}-\frac{\omega_{*_{e}}}{\omega}\left(1+2 \eta_{i}\right) \frac{e \Psi}{T_{e}}\right) n_{0} T_{i}
\end{aligned}
$$

\section{The Field Equations}

The set of equations is completed by using the quasi-neutrality condition (to higher order than introduced in the previous Section) and the parallel Ampère's law, as in Connor et al. (Connor et al. 2009). These will only be briefly discussed here, focussing on any differences from the work of Connor et al. (Connor et al. 2009) arising from the more complete description of the electron and ion continuity equations in the previous Sections and, effectively, a novel calculation of the neoclassical angular toroidal viscosity.

A convenient approach to imposing quasi-neutrality in higher order is through the vorticity equation, obtained by taking the charge density moment of the gyro-kinetic equations for both species and adding them. Setting $\tilde{n}_{e}=\tilde{n}_{i}$ then provides one relationship between $\Phi$ and $\Psi$. The parallel Ampère's law provides a second and hence these two equations lead to an eigenvalue condition on $\omega$, provided the solutions of the various continuity equations for $\tilde{n}, \quad \tilde{T}_{e} \quad$ and $\quad \tilde{T}_{i}$ are expressed in terms of $\Phi$ and $\Psi$. 


\section{(a) The Parallel Ampère's Law}

The parallel Ampère's Law states that

$$
|\nabla \chi|^{2} \frac{\mathrm{d}^{2} A_{\|}}{\mathrm{d} x^{2}}=\mu_{0} j_{\|}
$$

We expand eqn. (119) in the localisation, $x$, expressing $A_{\|}$in terms of $\Psi$ as

defined above eqn. (9), which we expand in the form $\Psi=\Psi^{(0)}+\Psi^{(1)}+\ldots \quad$ In leading-order we have

$$
\frac{|\nabla \chi|^{2}}{\mathrm{i} \omega} \frac{I}{R^{2} B q} \frac{\mathrm{d}^{2}}{\mathrm{~d} x^{2}} \frac{\partial}{\partial \theta} \Psi^{(0)}=0 \Rightarrow \Psi^{(0)}=\Psi^{(0)}(x)
$$

while in next order

$$
-\frac{|\nabla \chi|^{2}}{\mathrm{i} \omega} \frac{I}{R^{2} B q} \frac{\mathrm{d}^{2}}{\mathrm{~d} x^{2}}\left(\frac{\partial}{\partial \theta} \Psi^{(1)}+\mathrm{i} \ell q^{\prime} x \Psi^{(0)}(x)\right)=\mu_{0} j_{\|},
$$

leading to the solubility condition

$$
\frac{\ell q^{\prime} I}{\omega q}\left\langle\frac{1}{R^{2}}\right\rangle \frac{\mathrm{d}^{2}}{\mathrm{~d} x^{2}}\left(x \Psi^{(0)}\right)=-\mu_{0}\left(\frac{j_{\|} B}{|\nabla \chi|^{2}}\right\rangle,
$$

where, from eqn. (95), 


$$
\begin{gathered}
\left\langle\frac{j_{\|} B}{|\nabla \chi|^{2}}\right\rangle=-I \tilde{p}^{\prime}\left(\left\langle\frac{1}{|\nabla \chi|^{2}}\right\rangle-\left\langle\frac{B^{2}}{|\nabla \chi|^{2}}\right\rangle \frac{1}{\left\langle B^{2}\right\rangle}\right)+1.97 \tau_{e i} \frac{\mathrm{i} I \ell q^{\prime} x p_{0 e} e}{m_{e} q}\left\langle\frac{B^{2}}{|\nabla \chi|^{2}}\right\rangle \frac{1}{\left\langle B^{2}\right\rangle}\left\langle\frac{1}{R^{2}}\right\rangle \\
\times\left[\left(1-1.31 f_{t}\right)\left(\frac{\tilde{p}}{p_{0 e}}-\frac{e(\Phi-\Psi)}{T_{e}}-\frac{\omega_{*_{e}}}{\omega}\left(1+\eta_{e}\right) \frac{e \Psi}{T_{e}}\right)+0.34\left(1-0.69 f_{t}\right)\left(\frac{\tilde{T}_{e}}{T_{e}}-\frac{\omega_{* e} \eta_{e}}{\omega} \frac{e \Psi}{T_{e}}\right)\right] . \\
-\frac{f_{t} I p_{0 e}}{\left\langle B^{2}\right\rangle}\left\langle\frac{B^{2}}{|\nabla \chi|^{2}}\right\rangle\left[1.67 \frac{\tilde{p}^{\prime}}{p_{0 e}}-1.19 \frac{\tilde{T}_{e}^{\prime}}{T_{e}}-1.95 \frac{\tilde{T}_{i}^{\prime}}{T_{e}}\right]
\end{gathered}
$$

\section{(b) The Vorticity Equation}

The vorticity equation was obtained in the previous publication (Connor et al. 2009). Here we list the key steps in its derivation and quote the final result. The procedure was to add the velocity moments of the gyro-kinetic equations for the electrons and ions, take the long wavelength limit: $k_{\perp}^{2} \rho_{\mathrm{i}}^{2}<<1$ and exploit the lowest order quasi-neutrality condition: $\tilde{n}_{e}=\tilde{n}_{i}$, to obtain

$$
\begin{aligned}
& \frac{\boldsymbol{B} \cdot \nabla}{\omega^{2}}\left(\frac{|\nabla \chi|^{2}}{\mu_{0} B^{2}} \frac{\mathrm{d}^{2}}{\mathrm{~d} x^{2}} \boldsymbol{B} \cdot \nabla \Psi\right)= \\
& \quad \sum_{j} \frac{e_{j}^{2}}{T_{j}} \int \mathrm{d}^{3} \boldsymbol{v} f_{0 j}\left\{\frac{\omega_{*_{j}}^{T}}{\omega} \Phi-\left(1-\frac{\omega_{*_{j}}^{T}}{\omega}\right)\left[\frac{m_{j}^{2} v_{\perp}^{2}|\nabla \chi|^{2}}{2 e_{j}^{2} B^{2}} \frac{\mathrm{d}^{2}}{\mathrm{~d} x^{2}} \Phi+\frac{m_{j} v_{\perp}^{2}}{2 e_{j} B} \delta B_{\|}\right]\right\}, \\
& \quad+\mathrm{i} \sum_{j} e_{j} \int \mathrm{d}^{3} v \frac{v_{d j} \cdot \nabla g_{j}}{\omega}+\frac{1}{4 \omega} \sum_{j} e_{j} \int \mathrm{d}^{3} v \frac{L_{j}^{2} C_{j}\left(g_{j} L_{j}^{2}\right)}{2} \\
& \quad-\frac{\mathrm{i}}{\omega} \sum_{j} \frac{m_{j}}{e_{j}} \int \mathrm{d}^{3} \boldsymbol{v} \sigma\left|v_{\|}\right| \frac{\boldsymbol{B} \cdot \nabla}{B}\left(\frac{m_{j} v_{\perp}^{2}|\nabla \chi|^{2}}{2 B^{2}}\right) \frac{\mathrm{d}^{2}}{\mathrm{~d} x^{2}} g_{j}
\end{aligned}
$$

where the divergence of the parallel current has been expressed in terms of parallel gradients of $A_{\|}$, i.e. $\Psi$, through Ampère's law, eqn. (119). The 
distribution functions $g_{j}$ in the velocity space integrations over the magnetic drift terms are expressed in terms of the quantities $h_{j}$ and we note the final term vanishes for up-down symmetric equilibria. The expansions $\Psi=\Psi^{(0)}+\Psi^{(1)}+\Psi^{(2)} \ldots ; \quad h_{j}=h_{0 j}+h_{1 j}+h_{2 j} \ldots$. are introduced and the equation for $\Psi$ solved order by order.

In leading order one finds $\Psi^{(0)}$ is independent of $\theta$, while the equation for $\Psi^{(1)}$ :

$$
\frac{I^{2}}{\omega^{2} R^{2} q^{2}} \frac{\partial}{\partial \theta}\left[\frac{|\nabla \chi|^{2}}{\mu_{0} R^{2} B^{2}} \frac{\partial^{2}}{\partial x^{2}}\left(\frac{\partial}{\partial \theta} \Psi^{(1)}+\mathrm{i} \ell q^{\prime} x \Psi^{(0)}\right)\right]=\mathrm{i} \frac{I^{2}}{\omega R^{2} q} \frac{\partial}{\partial \theta}\left(\frac{\tilde{p}^{\prime}}{B^{2}}\right)
$$

can be integrated, introducing a constant of integration which can in turn be determined through a periodicity condition in $\theta$ on $\Psi^{(1)}$. Applying the same periodicity condition on $\Psi^{(2)}$ in second order provides the required equation for $\Psi^{(0)}(x):$ 


$$
\begin{aligned}
& \frac{I^{2}}{\omega^{2}}\left[\frac{\ell^{2} q^{\prime 2}}{\mu_{0} q^{2}}\left\langle\frac{1}{R^{2}}\right\rangle^{2} x \frac{\mathrm{d}^{2}\left(x \Psi^{(0)}\right)}{\mathrm{d} x^{2}}+\frac{\ell q^{\prime} x \omega}{q} \tilde{p}^{\prime}\left(\left\langle\frac{1}{B^{2} R^{2}}\right\rangle\left\langle\frac{B^{2}}{|\nabla \chi|^{2}}\right\rangle-\left\langle\frac{1}{R^{2}}\right\rangle\left\langle\frac{1}{|\nabla \chi|^{2}}\right\rangle\right)\right] \\
& =m_{i} n_{0}\left\langle\frac{|\nabla \chi|^{2}}{B^{2}}\right\rangle\left\langle\frac{B^{2}}{|\nabla \chi|^{2}}\right\rangle\left[1-\frac{\omega_{*_{i}}}{\omega}\left(1+\eta_{i}\right)\right] \frac{\mathrm{d}^{2} \Phi}{\mathrm{d} x^{2}} \\
& -2 \frac{\ell}{\omega} \tilde{p}\left\langle\frac{B^{2}}{|\nabla \chi|^{2}}\right\rangle\left\langle\frac{|\nabla \chi|^{2}}{R^{2} B^{2}} \frac{\partial}{\partial \chi}\left(\mu_{0} p_{0}+\frac{B^{2}}{2}\right)+\frac{\nabla \chi \cdot \nabla \theta}{R^{2} B^{2}} \frac{\partial B}{\partial \theta}\right\rangle \\
& \left.-\mathrm{i} \frac{I}{\omega}\left\langle\frac{B^{2}}{|\nabla \chi|^{2}}\right\rangle\left\langle\sum_{j} \int \mathrm{d}^{3} v \frac{m_{j} v_{\|}}{B} \frac{I}{R^{2} q} \frac{\partial}{\partial \theta}\left(\frac{v_{\|}}{B}\right) h_{j}^{\prime}\right\rangle-\frac{\ell p_{0}^{\prime} I^{2}}{\omega} \tilde{p}\left[\frac{1}{|\nabla \chi|^{2} B^{2}}\right\rangle\left\langle\frac{B^{2}}{|\nabla \chi|^{2}}\right\rangle-\left\langle\frac{1}{|\nabla \chi|^{2}}\right\rangle^{2}\right] \\
& -\frac{\ell^{2} q}{q^{\prime} p_{0}^{\prime} I^{2}} \\
& \omega^{2} q
\end{aligned}
$$

where we have substituted for $\delta B_{\|}$from eqn. (58) (which has the effect of replacing the $\nabla B$ drift by the curvature drift) and substituted for $\Psi^{(1)}$ from the solution of eqn. (125). The term involving $\left\langle\sum_{j} e_{j} \int \mathrm{d}^{3} v \overline{L_{j}^{2} C_{j}\left(g_{0 j} L_{j}^{2}\right)}\right\rangle$ represents the contribution from the classical radial transport of toroidal momentum, calculated in eqn. (B.2) of Appendix B.

It remains to evaluate the term involving the $h_{j}$, which we do by repeated application of the gyro-kinetic equations for $h_{0 j}, h_{1 j}, h_{2 j}$, and $h_{3 j}$ with integrations by parts in $\theta$ and noting momentum conservation in ion-ion collisions. The result can be expressed as 


$$
\begin{aligned}
& \mathrm{i} \frac{I}{\omega}\left\langle\sum_{j} \int \mathrm{d}^{3} \boldsymbol{v} \frac{m_{j} v_{\|}}{B} \frac{I}{R^{2} q} \frac{\partial}{\partial \theta}\left(\frac{v_{\|}}{B}\right) h_{j}^{\prime}\right\rangle \\
& \quad=\mathrm{i} \frac{I}{\omega}\left\langle\sum_{j} \int \mathrm{d}^{3} \boldsymbol{v} \frac{I m_{j} v_{\|}}{B}\left\{-\frac{I v_{\|}}{R^{2} B q} \frac{\partial}{\partial \theta}\left(\frac{v_{\|}}{\Omega_{i}}\right) h_{j}^{\prime \prime}+C_{j}\left(h_{j}^{\prime}\right)+\mathrm{i} \omega h_{j}^{\prime}-\mathrm{i} \ell q^{\prime} \frac{I v_{\|}}{R^{2} B q}\left(x h_{j}^{\prime}\right)\right\}\right\rangle
\end{aligned}
$$

The first term can be recognised as involving the neoclassical radial transport of toroidal angular momentum:

$$
\left\langle\Pi^{N C}\right\rangle=\left\langle\sum_{j} \int \mathrm{d}^{3} v v m_{j} \frac{I}{B} v_{\|} v_{d r j} h_{j}\right\rangle,
$$

where the first non-vanishing contribution is from $h_{3 j}$. The right-hand-side of expression (127) thus reduces to

$$
-\frac{\mathrm{i}}{\omega}\left\langle\Pi^{N C}\right\rangle^{\prime \prime}-I m_{i} n_{0}\left\langle\frac{U_{\| i}}{B}\right\rangle^{\prime}+\frac{\ell q^{\prime} I^{2}}{\omega q}\left\langle\frac{1}{R^{2} B^{2}}\right\rangle\left[x\left(\tilde{p}-\frac{\ell}{\omega} p_{0}^{\prime} \Psi^{(0)}\right)^{\prime}\right] .
$$

Further use of the gyro-kinetic equation and integrations by parts in $\theta$ implies

$$
-\frac{\mathrm{i}}{\omega}\left\langle\Pi^{N C}\right\rangle^{\prime \prime}=\mathrm{i} \frac{I}{2 \omega}\left\langle\sum_{j} \int \mathrm{d}^{3} \boldsymbol{v} \frac{m_{j} v_{\|}}{B} \frac{I v_{\|}}{\Omega_{j}}\left[C_{j}\left(h_{2 j}^{\prime \prime}\right)+\frac{I v_{\|}}{3 \Omega_{j}} C_{j}\left(h_{1 j}^{\prime \prime}\right)\right]\right\rangle .
$$

Assembling all the contributions to eqn. (126) and substituting for $U_{\| i}$ from eqn. (36), 


$$
\begin{aligned}
x \frac{\mathrm{d}^{2}}{\mathrm{~d} x^{2}}\left(x \Psi^{(0)}\right)+\left(D_{I}+\frac{1}{4}\right) \frac{\omega}{\ell p_{0}^{\prime}} \tilde{p}-(L+H) x \frac{\mathrm{d}}{\mathrm{d} x}\left(\frac{\omega}{\ell p_{0}^{\prime}} \tilde{p}-\Psi^{(0)}\right) \\
\left.\quad=\frac{\mu_{0} m_{i} n_{0}}{\ell^{2}}\left(\frac{q}{I q^{\prime}\left\langle 1 / R^{2}\right\rangle}\right)^{2}\left\langle\frac{B^{2}}{|\nabla \chi|^{2}}\right\rangle \frac{|\nabla \chi|^{2}}{B^{2}}\right\rangle \omega\left(\omega-\omega_{*_{i}}\left(1+\eta_{i}\right)\right) \frac{\mathrm{d}^{2}}{\mathrm{~d} x^{2}} \Phi \\
+\frac{\mu_{0} m_{i} n_{0}}{\ell^{2}}\left(\frac{q}{q^{\prime}\left\langle 1 / R^{2}\right\rangle}\right)^{2} \frac{\omega^{2} T_{i}}{e}\left\langle\frac{B^{2}}{|\nabla \chi|^{2}}\right\rangle \frac{\mathrm{d}^{2}}{\mathrm{~d} x^{2}}\left\{\left\langle\frac{1}{B^{2}}\right\rangle\left(\frac{\tilde{n}}{n_{0}}+\frac{e \Phi}{T_{i}}\right)+\left(\left\langle\frac{1}{B^{2}}\right\rangle-\frac{1.17}{\left\langle B^{2}\right\rangle}\left(1-f_{t}\right)\right) \frac{\tilde{T}_{i}}{T_{i}}\right\} \\
\quad-\frac{i \omega \mu_{0}}{\ell^{2}}\left(\frac{q}{I q^{\prime}\left\langle 1 / R^{2}\right\rangle}\right)^{2}\left\langle\frac{B^{2}}{|\nabla \chi|^{2}}\right\rangle \frac{\mathrm{d}^{2}}{\mathrm{~d} x^{2}}\left\langle\Pi^{C}+\Pi^{N C}\right\rangle
\end{aligned}
$$

Here we have introduced the quantity $D_{I}=E+F+H$, in the notation of Glasser et al. (Glasser et al. 1975), and L, appearing in the work of Hahm (Hahm 1988), where

$$
L=\left(\frac{\mu_{0} p_{0}^{\prime} q}{q^{\prime}\left\langle 1 / R^{2}\right\rangle\left\langle B^{2}\right\rangle}\right)\left\langle\frac{B^{2}}{|\nabla \chi|^{2}}\right\rangle
$$

The quantity $D_{I}$ plays a role in the Mercier stability criterion: $D_{I}+1 / 4>0$ (Mercier 1960) while the combination $L+H$ in eqn. (131) is given by

$$
L+H=\left(\frac{\mu_{0} p_{0}^{\prime} q}{q^{\prime}\left\langle 1 / R^{2}\right\rangle}\right)\left\langle\frac{1}{|\nabla \chi|^{2}}\right\rangle
$$

An expression for $\left\langle\Pi^{N C}\right\rangle$ has been given by Wong and Chan (Wong \& Chan 2005): $\left\langle\Pi^{N C}\right\rangle=0.19\left(n_{0} m_{i}^{2} T_{i} f_{t}^{3} / \tau_{i i} e^{3}\right) \mathrm{d}^{2} \tilde{T}_{i} / \mathrm{d} x^{2}$, where $\tau_{i i}=\tau_{i} / \sqrt{2}$, but we 
generalise this in Appendix B for arbitrary axisymmetric toroidal geometry, to obtain the total collisional toroidal angular viscosity:

$$
\langle\Pi\rangle=\left\langle\Pi^{C}\right\rangle+\left\langle\Pi^{N C}\right\rangle
$$

where

$$
\left\langle\Pi^{C}\right\rangle=-\frac{n_{0} e}{5 \tau_{i}}\left(\frac{2 m_{i} T_{i}}{e^{2}}\right)^{2}\left\langle\frac{|\nabla \chi|^{4}}{B^{4}}\right\rangle \frac{\mathrm{d}^{2}}{\mathrm{~d} x^{2}}\left\{\frac{\tilde{p}_{i}}{p_{0 i}}+\frac{e \Phi}{T_{i}}+3 \frac{\tilde{T}_{i}}{T_{i}}\right\},
$$

and

$$
\begin{aligned}
& \left\langle\Pi^{N C}\right\rangle=0.80 \frac{n_{0} e}{\tau_{i}}\left(\frac{I^{2} m_{i} T_{i}}{e^{2}}\right)^{2}\left[\left\langle\frac{1}{B^{4}}\right\rangle+\frac{\left(G^{(3)}-G^{(2)}\right)}{\left\langle B^{2}\right\rangle^{2}}\right] \frac{\mathrm{d}^{2}}{\mathrm{~d} x^{2}}\left(\frac{\tilde{p}_{i}}{p_{0 i}}+\frac{e \Phi}{T_{i}}\right) \\
& +\frac{n_{0} e}{\tau_{i}\left\langle B^{2}\right\rangle}\left(\frac{I^{2} m_{i} T_{i}}{e^{2}}\right)^{2}\left[0.37\left(\frac{1}{\left\langle B^{2}\right\rangle}\left\langle\frac{1}{B^{2}}\right\rangle-\left\langle\frac{1}{B}\right\rangle\left\langle\frac{1}{B^{3}}\right\rangle\right)+0.54\left(\frac{1}{B^{4}}\right\rangle\right] \frac{\mathrm{d}^{2}}{\mathrm{~d} x^{2}}\left(\frac{\tilde{T}_{i}}{T_{i}}\right) \\
& +\frac{n_{0} e}{\tau_{i}\left\langle B^{2}\right\rangle^{2}}\left(\frac{I^{2} m_{i} T_{i}}{e^{2}}\right)^{2}\left(0.37 G^{(0)}-0.62 G^{[1]}+0.94 G^{(2)}-0.2 G^{(3)}-0.74 G^{(4)}\right) \frac{\mathrm{d}^{2}}{\mathrm{~d} x^{2}}\left(\frac{\tilde{T}_{i}}{T_{i}}\right)
\end{aligned}
$$

with

$$
\begin{gathered}
G^{(0)}=\frac{15\left\langle B^{2}\right\rangle^{2}}{4}\left\langle\frac{1}{B^{2}} \int_{0}^{1 / B_{\text {Max }}} \mathrm{d} \lambda \frac{\lambda(1-\lambda B)}{\langle\sqrt{1-\lambda B}\rangle}\right\rangle, \\
\frac{G^{(1)}}{3\left\langle B^{2}\right\rangle}=\frac{15}{4} \int_{0}^{1 / B_{\text {Min }}} \mathrm{d} \lambda \lambda \frac{\langle B \sqrt{1-\lambda B}\rangle}{\langle\sqrt{1-\lambda B}\rangle}\left\langle\frac{\sqrt{1-\lambda B}}{B}\right\rangle+\frac{15}{16}\left\langle B^{2}\right\rangle\left\langle\frac{1}{B}\right\rangle_{0}^{1 / B_{\text {Max }}} \frac{\mathrm{d} \lambda \lambda^{2}}{\langle\sqrt{1-\lambda B}\rangle}, \\
-\frac{15}{8}\left\langle B^{2}\right\rangle \int_{0}^{1 / B_{\text {Max }}} \mathrm{d} \lambda \lambda \frac{1}{\langle\sqrt{1-\lambda B}\rangle}\left\langle\frac{\sqrt{1-\lambda B}}{B}\right\rangle \int_{\lambda}^{1 / B_{\text {Max }}} \frac{\mathrm{d} \lambda^{\prime}}{\left\langle\sqrt{1-\lambda^{\prime} B}\right\rangle}
\end{gathered}
$$




$$
\begin{aligned}
& G^{(2)}=\frac{15\left\langle B^{2}\right\rangle^{2}}{4}\left\langle\frac{1}{B^{2}} \int_{0}^{1 / B_{\text {Max }}} \lambda \mathrm{d} \lambda \frac{\langle B \sqrt{1-\lambda B}\rangle}{\langle\sqrt{1-\lambda B}\rangle}\left\langle\frac{\sqrt{1-\lambda B}}{B}\right\rangle\right\rangle, \\
& G^{(3)}=\frac{15\left\langle B^{2}\right\rangle^{2}}{4} \int_{0}^{1 / B_{\text {Min }}} \mathrm{d} \lambda \lambda \frac{1}{\langle\sqrt{1-\lambda B}\rangle}\left\langle\frac{\sqrt{1-\lambda B}}{B}\right\rangle\left(\frac{\langle B \sqrt{1-\lambda B}\rangle}{\left\langle B^{2}\right\rangle}-\left\langle\frac{\sqrt{1-\lambda B}}{B}\right\rangle\right)
\end{aligned}
$$

and

$$
G^{(4)}=\frac{15\left\langle B^{2}\right\rangle^{2}}{4} \int_{0}^{1 / B_{M a x}} \mathrm{~d} \lambda \lambda \frac{1}{\langle\sqrt{1-\lambda B}\rangle}\left(\frac{1}{\langle\sqrt{1-\lambda B}\rangle}\left\langle\frac{\sqrt{1-\lambda B}}{B}\right\rangle\left\langle\frac{1-\lambda B}{B}\right\rangle-\left\langle\frac{1-\lambda B}{B^{2}}\right\rangle\right)
$$

Insertion of the results (135) and (136) completes the form of the vorticity equation (131). Analytic evaluations of the coefficients in eqn. (136) in the large aspect ratio limit are presented in Appendix B and are consistent with those given by Wong and Chan (Wong \& Chan 2005).

\section{Normalised Equations}

It is convenient to introduce a new radial co-ordinate normalised to the semicollisional width, $\delta_{e}$ (but now expressed in terms of flux co-ordinates) and a set of normalised parameters

$$
s=\mathrm{e}^{\mathrm{i} \pi / 4} \frac{\ell q^{\prime}}{q}\left(1.97 \frac{T_{e}}{m_{e}} \frac{\tau_{e i}}{\omega}\left\langle\frac{1}{R^{2}}\right\rangle^{2} \frac{I^{2}}{\left\langle B^{2}\right\rangle}\right)^{1 / 2} x \equiv \frac{x}{\delta_{e}} .
$$




$$
\begin{gathered}
\hat{\omega}=\frac{e n_{0}}{\ell T_{e} n_{0}^{\prime}} \omega, \quad \varphi=\frac{e \Phi}{T_{e}}, \psi=\frac{e \Psi}{T_{e}}, \quad n=\frac{\tilde{n}}{n_{0}}, t_{e}=\frac{\tilde{T}_{e}}{T_{e}}, t_{i}=\frac{\tilde{T}_{i}}{T_{e}}, \\
p_{j}=\frac{\tilde{p}_{j}}{p_{0 e}}, \quad p=\frac{\tilde{p}}{p_{0 e}}, \quad \kappa=\frac{q^{\prime}}{q} \frac{n_{0}}{n_{0}^{\prime}}, \\
C=\frac{q}{q^{\prime}}\left\langle\left(\frac{1}{B^{2}} \frac{\partial}{\partial \chi}\left(\mu_{0} p_{0}+B^{2}\right)+\left(\frac{1}{R B^{2}}\right)^{2} \nabla \chi \cdot \nabla \theta \frac{\partial B^{2}}{\partial \theta}\right)\right\rangle
\end{gathered}
$$

where $\delta_{e}$ is the semi-collisional width in flux coordinates. As a consequence, the normalised electron continuity equation (116) becomes:

$$
\begin{aligned}
& n-\frac{\varphi}{\hat{\omega}}+\left\langle\frac{\mu_{0} p_{0 e}}{B^{2}}\right\rangle p\left(1-\frac{1+\eta_{e}}{\hat{\omega}}\right)-\frac{\kappa}{\hat{\omega}} C\left(p_{e}-(\varphi-\psi)-\frac{1+\eta_{e}}{\hat{\omega}} \psi\right) \\
& +s^{2}\left[\left(1-1.31 f_{t}\right)\left(p_{e}-(\varphi-\psi)-\frac{1+\eta_{e}}{\hat{\omega}} \psi\right)+0.34\left(1-0.69 f_{t}\right)\left(t_{e}-\frac{\eta_{e}}{\hat{\omega}} \psi\right)\right] \\
& -\frac{\kappa s}{\hat{\omega}} \frac{\mathrm{d}}{\mathrm{d} s}\left[\left(\left\langle\frac{I^{2}}{R^{2} B^{2}}\right\rangle-\frac{1}{\left\langle B^{2}\right\rangle}\left\langle\frac{I^{2}}{R^{2}}\right\rangle\right) p+\frac{f_{t}}{\left\langle B^{2}\right\rangle}\left\langle\frac{I^{2}}{R^{2}}\right\rangle\left(1.67 p-1.19 t_{e}-3.12 t_{i}\right)-\left\langle\frac{I^{2}}{R^{2} B^{2}}\right\rangle\left(p_{i}+\varphi\right)+\frac{1.17}{\left\langle B^{2}\right\rangle}\left\langle\frac{I^{2}}{R^{2}}\right\rangle t_{i}\right] \\
& +\frac{\kappa}{\hat{\omega}}\left(\left\langle\frac{I^{2}}{R^{2} B^{2}}\right\rangle-\frac{1}{\left\langle B^{2}\right\rangle}\left\langle\frac{I^{2}}{R^{2}}\right\rangle\right) \frac{\mathrm{d}}{\mathrm{d} s}\left[s\left(p_{e}-(\varphi-\psi)-\frac{1+\eta_{e}}{\hat{\omega}} \psi\right)\right] \\
& +1.67 f_{t} \frac{\kappa}{\hat{\omega}} \frac{1}{\left\langle B^{2}\right\rangle}\left\langle\frac{I^{2}}{R^{2}}\right\rangle \frac{\mathrm{d}}{\mathrm{d} s}\left[s\left(p_{e}-(\varphi-\psi)-\frac{1+1.02 \eta_{e}}{\hat{\omega}} \psi+0.02 t_{e}\right)\right]+1.97\left(\frac{\kappa}{\hat{\omega}}\right)^{2} \frac{1}{\left\langle B^{2}\right\rangle}\left\langle\frac{I^{2}}{R^{2}}\right\rangle \\
& \times \frac{\mathrm{d}^{2}}{\mathrm{~d} s^{2}}\left[\left(\left\langle\frac{|\nabla \chi|^{2}}{B^{2}}\right\rangle\left\langle\frac{1}{R^{2}}\right\rangle+\left\langle\frac{I^{2}}{R^{2}}\right\rangle\left(\left\langle\frac{1}{B^{2}}\right\rangle-\frac{1}{\left\langle B^{2}\right\rangle}\right)\right)\left(p-\frac{3}{2} t_{e}\right)+\frac{1.53 f_{t}}{\left\langle B^{2}\right\rangle}\left\langle\frac{I^{2}}{R^{2}}\right\rangle\left(p+1.39 t_{e}-1.17 t_{i}\right)\right]=0,
\end{aligned}
$$

the electron thermal equation (117): 


$$
\begin{aligned}
& \frac{3}{2}\left[p_{e}-\frac{1+\eta_{e}}{\hat{\omega}} \varphi+\frac{5}{3}\left\langle\frac{\mu_{0} p_{0 e}}{B^{2}}\right\rangle p\left(1-\frac{1+2 \eta_{e}}{\hat{\omega}}\right)\right]-\frac{5}{2} \frac{\kappa}{\hat{\omega}} C\left(p_{e}+t_{e}-(\varphi-\psi)-\frac{1+2 \eta_{e}}{\hat{\omega}} \psi\right) \\
& +3.18 s^{2}\left[\left(1-1.11 f_{t}\right)\left(p_{e}-(\varphi-\psi)-\frac{1+\eta_{e}}{\hat{\omega}} \psi\right)+0.58\left(1-0.31 f_{t}\right)\left(t_{e}-\frac{\eta_{e}}{\hat{\omega}} \psi\right)\right] \\
& -\frac{5}{2} \frac{\kappa}{\hat{\omega}}\left(\left\langle\frac{I^{2}}{R^{2} B^{2}}\right\rangle-\frac{1}{\left\langle B^{2}\right\rangle}\left\langle\frac{I^{2}}{R^{2}}\right\rangle\right) s \frac{\mathrm{d}}{\mathrm{d} s}\left(p+t_{e}\right)-\frac{\kappa}{\hat{\omega}} \frac{f_{t}}{\left\langle B^{2}\right\rangle}\left\langle\frac{I^{2}}{R^{2}}\right\rangle s \frac{\mathrm{d}}{\mathrm{d} s}\left(4.18 p-2.36 t_{e}-7.86 t_{i}\right) \\
& +\frac{5}{2} \frac{\kappa}{\hat{\omega}} s \frac{\mathrm{d}}{\mathrm{d} s}\left[\left\langle\frac{I^{2}}{R^{2} B^{2}}\right\rangle\left(p_{i}+\varphi\right)-\frac{1.17}{\left\langle B^{2}\right\rangle}\left\langle\frac{I^{2}}{R^{2}}\right\rangle t_{i}\right] \\
& +\frac{5}{2} \frac{\kappa}{\hat{\omega}}\left(\left\langle\frac{I^{2}}{R^{2} B^{2}}\right\rangle-\frac{1}{\left\langle B^{2}\right\rangle}\left\langle\frac{I^{2}}{R^{2}}\right\rangle\right) \frac{\mathrm{d}}{\mathrm{d} s}\left[s\left(p_{e}-(\varphi-\psi)-\frac{1+2 \eta_{e}}{\hat{\omega}} \psi+t_{e}\right)\right] \\
& +2.97 f_{t} \frac{\kappa}{\hat{\omega}} \frac{1}{\left\langle B^{2}\right\rangle}\left\langle\frac{I^{2}}{R^{2}}\right) \frac{\mathrm{d}}{\mathrm{d} s}\left[s\left(p_{e}-(\varphi-\psi)-\frac{1+1.25 \eta_{e}}{\hat{\omega}} \psi+0.25 t_{e}\right)\right]+1.97\left(\frac{\kappa}{\hat{\omega}}\right)^{2} \frac{1}{\left\langle B^{2}\right\rangle}\left\langle\frac{I^{2}}{R^{2}}\right\rangle \\
& \times \frac{\mathrm{d}^{2}}{\mathrm{~d} s^{2}}\left[\left(\left\langle\frac{|\nabla \chi|^{2}}{B^{2}}\right\rangle\left\langle\frac{1}{R^{2}}\right\rangle+\left\langle\frac{I^{2}}{R^{2}}\right\rangle\left(\left\langle\frac{1}{B^{2}}\right\rangle-\frac{1}{\left\langle B^{2}\right\rangle}\right)\right)\left(p+0.91 t_{e}\right)+\frac{1.70 f_{t}}{\left\langle B^{2}\right\rangle}\left\langle\frac{I^{2}}{R^{2}}\right\rangle\left(p-0.4 t_{e}-1.17 t_{i}\right)\right]=0,
\end{aligned}
$$

the ion thermal equation (118):

$$
\begin{aligned}
& \frac{3}{2}\left(p_{i}-\frac{1+\eta_{i}}{\hat{\omega}} \frac{T_{i}}{T_{e}} \varphi+\frac{5}{3}\left\langle\frac{\mu_{0} p_{0 e}}{B^{2}}\right\rangle\left(1+\frac{T_{i}}{T_{e}} \frac{1+2 \eta_{i}}{\hat{\omega}}\right)\right) \\
& +\frac{5}{2} \frac{T_{i}}{T_{e}} \frac{\kappa}{\hat{\omega}} C\left(p_{i}+t_{i}+(\varphi-\psi)-\frac{1+2 \eta_{i}}{\hat{\omega}} \frac{T_{i}}{T_{e}} \psi\right)+3.94\left(\frac{\kappa}{\hat{\omega}}\right)^{2} \frac{m_{i} \tau_{e i}}{m_{e} \tau_{i}} \frac{T_{i}}{T_{e}} \frac{1}{\left\langle B^{2}\right\rangle}\left\langle\frac{I^{2}}{R^{2}}\right\rangle \\
& \times \frac{\mathrm{d}^{2}}{\mathrm{~d} s^{2}}\left[\left(\left\langle\frac{|\nabla \chi|^{2}}{B^{2}}\right\rangle\left\langle\frac{1}{R^{2}}\right\rangle+\left\langle\frac{I^{2}}{R^{2}}\right\rangle\left(\left\langle\frac{1}{B^{2}}\right\rangle-\frac{1}{\left\langle B^{2}\right\rangle}\right)\right)+\frac{0.46 f_{t}}{\left\langle B^{2}\right\rangle}\left\langle\frac{I^{2}}{R^{2}}\right\rangle\right] t_{i}=0
\end{aligned}
$$

Ampère's Law (122), with result (123) for $j_{\|}$: 


$$
\begin{aligned}
& \frac{\mathrm{d}^{2}}{\mathrm{~d} s^{2}}(s \psi)=\frac{\mu_{0} p_{0 e}}{\left\langle R^{-2}\right\rangle} \frac{\hat{\omega}}{\kappa} \\
& \quad \times\left[\left(\left\langle\frac{1}{|\nabla \chi|^{2}}\right\rangle-\frac{1}{\left\langle B^{2}\right\rangle}\left\langle\frac{B^{2}}{|\nabla \chi|^{2}}\right\rangle\right) \frac{\mathrm{d} p}{\mathrm{~d} s}+\frac{f_{t}}{\left\langle B^{2}\right\rangle}\left\langle\frac{B^{2}}{|\nabla \chi|^{2}}\right\rangle\left(1.67 \frac{\mathrm{d} p}{\mathrm{~d} s}-1.19 \frac{\mathrm{d} t_{e}}{\mathrm{~d} s}-1.95 \frac{\mathrm{d} t_{i}}{\mathrm{~d} s}\right)\right] \\
& -\left(\frac{\hat{\omega}}{\kappa}\right)^{2} \frac{\mu_{0} p_{0 e}}{I^{2}\left\langle R^{-2}\right\rangle^{2}}\left\langle\frac{B^{2}}{|\nabla \chi|^{2}}\right\rangle s\left[\left(1-1.31 f_{t}\right)\left(p_{e}-(\varphi-\psi)-\frac{1+\eta_{e}}{\hat{\omega}} \psi\right)+0.34\left(1-0.66 f_{t}\right)\left(t_{e}-\frac{\eta_{e}}{\hat{\omega}} \psi\right)\right],
\end{aligned}
$$

and the vorticity equation (131):

$$
\begin{aligned}
& s \frac{\mathrm{d}^{2}}{\mathrm{~d} s^{2}}(s \psi)+\left(D_{I}+\frac{1}{4}\right)\left(\frac{T_{e} n_{0}^{\prime}}{p_{0}^{\prime}}\right) \hat{\omega} p-(L+H) s \frac{\mathrm{d}}{\mathrm{d} s}\left(\frac{T_{e} n_{0}^{\prime}}{p_{0}^{\prime}} \hat{\omega} p-\psi\right) \\
& \left.=1.97 \mathrm{i} \frac{\mu_{0} p_{0 e}}{\left\langle B^{2}\right\rangle} \frac{m_{i}}{m_{e}}\left(\frac{\ell T_{e} n_{0}^{\prime}}{e n_{0}} \tau_{e i}\right)\left\langle\frac{B^{2}}{|\nabla \chi|^{2}}\right\rangle \frac{|\nabla \chi|^{2}}{B^{2}}\right\rangle \hat{\omega}\left(1+\frac{T_{i}}{\hat{\omega} T_{e}}\left(1+\eta_{i}\right)\right) \frac{\mathrm{d}^{2}}{\mathrm{~d} s^{2}} \varphi \\
& +1.97 \mathrm{i} \frac{\mu_{0} p_{0 e}}{\left\langle B^{2}\right\rangle} \frac{m_{i}}{m_{e}}\left(\frac{\ell T_{e} n_{0}^{\prime}}{e n_{0}} \tau_{e i}\right)\left\langle\frac{B^{2}}{|\nabla \chi|^{2}}\right\rangle \hat{\omega} \frac{\mathrm{d}^{2}}{\mathrm{~d} s^{2}}\left[\left\langle\frac{I^{2}}{B^{2}}\right\rangle\left(\frac{T_{i}}{T_{e}} n+\varphi\right)+\left(\left\langle\frac{I^{2}}{B^{2}}\right\rangle-\frac{1.17 I^{2}}{\left\langle B^{2}\right\rangle}\left(1-f_{t}\right)\right) t_{i}\right] \\
& -(1.97)^{2} \frac{\mathrm{i}}{\hat{\omega}} \frac{\mu_{0} p_{0 e}}{\left\langle B^{2}\right\rangle} \frac{T_{i}}{T_{e}}\left(\frac{\tau_{e i}^{2}}{\tau_{i}} \frac{\ell T_{e} n_{0}^{\prime}}{e n_{0}}\right)\left(\frac{m_{i}}{m_{e} \kappa}\right)^{2} \frac{I^{2}}{\left\langle B^{2}\right\rangle}\left\langle\frac{B^{2}}{|\nabla \chi|^{2}}\right\rangle \frac{\mathrm{d}^{2}}{\mathrm{~d} s^{2}} \hat{\Pi},
\end{aligned}
$$

where

$$
\begin{aligned}
\frac{\mathrm{d}^{2}}{\mathrm{~d} s^{2}} \hat{\Pi}= & -0.80\left\langle\frac{|\nabla \chi|^{4}}{B^{4}}\right\rangle\left\langle\frac{1}{R^{2}}\right\rangle^{2} \frac{\mathrm{d}^{4}}{\mathrm{~d} s^{4}}\left(p_{i}+\varphi\right)+0.80\left\langle\frac{I^{2}}{R^{2}}\right\rangle^{2}\left[\left\langle\frac{1}{B^{4}}\right\rangle+\frac{\left(G^{(3)}-G^{(2)}\right)}{\left\langle B^{2}\right\rangle^{2}}\right] \frac{\mathrm{d}^{4}}{\mathrm{~d} s^{4}}\left(p_{i}+\varphi\right) \\
& +\left\langle\frac{I^{2}}{R^{2}}\right\rangle^{2}\left[0.37\left(\frac{1}{\left\langle B^{2}\right\rangle}\left\langle\frac{1}{B^{2}}\right\rangle-\left\langle\frac{1}{B}\right\rangle\left\langle\frac{1}{B^{3}}\right\rangle\right)+0.54\left\langle\frac{1}{B^{4}}\right\rangle-2.4\left\langle\frac{|\nabla \chi|^{4}}{I^{4} B^{4}}\right\rangle \frac{\mathrm{d}^{4} t_{i}}{\mathrm{~d} s^{4}}\right. \\
& +\left(0.37 G^{(0)}-0.62 G^{(1)}+0.94 G^{(2)}-0.2 G^{(3)}-0.74 G^{(4)}\right) \frac{\mathrm{d}^{4} t_{i}}{\mathrm{~d} s^{4}}
\end{aligned}
$$


with $G^{(0)}, G^{(1)}, G^{(2)}, G^{(3)}$ and $G^{(4)}$ given in eqns. (137) - (141), respectively. Because the ion thermal conductivity exceeds that of the electrons by $O\left(\sqrt{m_{i} / m_{e}}\right)$, the ion temperature is flattened over the semi-collisional width and one can treat it as constant. In particular, the terms involving the ion temperature gradients in the classical and neoclassical toroidal viscosity (148) can be neglected, simplifying it considerably. It is only in the region $s \sim\left(m_{i} / m_{e}\right)^{1 / 4}$ that one needs to solve the ion thermal differential equation. As we shall see the electron equations simplify in this region of large $s$, thus simplifying the form of the ion thermal equation.

Equations (143) - (146) simplify if we ignore the classical and Pfirsch-Schlüter transport relative to the larger banana contribution. Furthermore, recognising that the azimuthal fluxes are small and that $\beta_{e}=2 \mu_{0} p_{0 e} / B^{2}<<1$, we obtain the following simpler set. The electron continuity equation (143) becomes:

$$
\begin{aligned}
& n-\frac{\varphi}{\hat{\omega}}+s^{2}\left[\left(1-1.31 f_{t}\right)\left(p_{e}-(\varphi-\psi)-\frac{1+\eta_{e}}{\hat{\omega}} \psi\right)+0.34\left(1-0.69 f_{t}\right)\left(t_{e}-\frac{\eta_{e}}{\hat{\omega}} \psi\right)\right] \\
& -\frac{\kappa s}{\hat{\omega}} \frac{\mathrm{d}}{\mathrm{d} s}\left[\frac{f_{t}}{\left\langle B^{2}\right\rangle}\left\langle\frac{I^{2}}{R^{2}}\right\rangle\left(1.67 p-1.19 t_{e}-3.12 t_{i}\right)-\left\langle\frac{I^{2}}{R^{2} B^{2}}\right\rangle\left(p_{i}+\varphi\right)+\frac{1.17}{\left\langle B^{2}\right\rangle}\left\langle\frac{I^{2}}{R^{2}}\right\rangle t_{i}\right] \\
& +1.67 f_{t} \frac{\kappa}{\hat{\omega}} \frac{1}{\left\langle B^{2}\right\rangle}\left\langle\frac{I^{2}}{R^{2}}\right\rangle \frac{\mathrm{d}}{\mathrm{d} s}\left[s\left(p_{e}-(\varphi-\psi)-\frac{1+1.02 \eta_{e}}{\hat{\omega}} \psi+0.02 t_{e}\right)\right] \\
& +3.01\left(\frac{\kappa}{\hat{\omega}}\right)^{2} \frac{f_{t}}{\left\langle B^{2}\right\rangle^{2}}\left\langle\frac{I^{2}}{R^{2}}\right\rangle^{2} \frac{\mathrm{d}^{2}}{\mathrm{~d}^{2}}\left(p+1.39 t_{e}-1.17 t_{i}\right)=0,
\end{aligned}
$$

the electron thermal equation (144) becomes: 


$$
\begin{aligned}
& \frac{3}{2}\left[p_{e}-\frac{1+\eta_{e}}{\hat{\omega}} \varphi\right]+3.18 s^{2}\left[\left(1-1.11 f_{t}\right)\left(p_{e}-(\varphi-\psi)-\frac{1+\eta_{e}}{\hat{\omega}} \psi\right)+0.58\left(1-0.31 f_{t}\right)\left(t_{e}-\frac{\eta_{e}}{\hat{\omega}} \psi\right)\right] \\
& -\frac{5}{2} \frac{\kappa}{\hat{\omega}} s \frac{\mathrm{d}}{\mathrm{d} s}\left[\frac{f_{t}}{\left\langle B^{2}\right\rangle}\left\langle\frac{I^{2}}{R^{2}}\right\rangle\left(4.18 p-2.36 t_{e}-7.86 t_{i}\right)-\left\langle\frac{I^{2}}{R^{2} B^{2}}\right\rangle\left(p_{i}+\varphi\right)+\frac{1.17}{\left\langle B^{2}\right\rangle}\left\langle\frac{I^{2}}{R^{2}}\right\rangle t_{i}\right] \\
& +2.97 f_{t} \frac{\kappa}{\hat{\omega}} \frac{1}{\left\langle B^{2}\right\rangle}\left\langle\frac{I^{2}}{R^{2}}\right\rangle \frac{\mathrm{d}}{\mathrm{d} s}\left[s\left(p_{e}-(\varphi-\psi)-\frac{1+1.25 \eta_{e}}{\hat{\omega}} \psi+0.25 t_{e}\right)\right] \\
& +3.35\left(\frac{\kappa}{\hat{\omega}}\right)^{2} \frac{f_{t}}{\left\langle B^{2}\right\rangle^{2}}\left\langle\frac{I^{2}}{R^{2}}\right\rangle^{2} \frac{\mathrm{d}^{2}}{\mathrm{~d} s^{2}}\left(p-0.4 t_{e}-1.17 t_{i}\right)=0
\end{aligned}
$$

the ion thermal equation (145) becomes:

$$
\frac{3}{2}\left(p_{i}-\frac{1+\eta_{i}}{\hat{\omega}} \frac{T_{i}}{T_{e}} \varphi\right)+1.81\left(\frac{\kappa}{\hat{\omega}}\right)^{2} \frac{m_{i} \tau_{e i}}{m_{e} \tau_{i}} \frac{T_{i}}{T_{e}} \frac{1}{\left\langle B^{2}\right\rangle^{2}}\left\langle\frac{I^{2}}{R^{2}}\right\rangle^{2} \frac{\mathrm{d}^{2}}{\mathrm{~d} s^{2}} t_{i}=0
$$

and Ampère's Law (146) becomes:

$$
\begin{aligned}
& \frac{\mathrm{d}^{2}}{\mathrm{~d} s^{2}}(s \psi)=\frac{\mu_{0} p_{0 e}}{\left\langle R^{-2}\right\rangle} \frac{\hat{\omega}}{\kappa} \frac{f_{t}}{\left\langle B^{2}\right\rangle}\left\langle\frac{B^{2}}{|\nabla \chi|^{2}}\right\rangle\left(1.67 \frac{\mathrm{d} p}{\mathrm{~d} s}-1.19 \frac{\mathrm{d} t_{e}}{\mathrm{~d} s}-1.95 \frac{\mathrm{d} t_{i}}{\mathrm{~d} s}\right) \\
& \left.-\left(\frac{\hat{\omega}}{\kappa}\right)^{2} \frac{\mu_{0} p_{0 e}}{I^{2}\left\langle R^{-2}\right\rangle^{2}}\left\langle\frac{B^{2}}{|\nabla \chi|^{2}}\right\rangle s \mid\left(1-1.31 f_{t}\right)\left(p_{e}-(\varphi-\psi)-\frac{1+\eta_{e}}{\hat{\omega}} \psi\right)+0.34\left(1-0.66 f_{t}\right)\left(t_{e}-\frac{\eta_{e}}{\hat{\omega}} \psi\right)\right],
\end{aligned}
$$

These approximations do not affect the vorticity equation (147). 


\section{Boundary Conditions and an Intermediate Region}

The purpose in solving the above layer equations is to match solutions of a given parity at $s=0$ to the marginal ideal MHD solutions at large $s$, which involve the tearing mode parameter, $\Delta^{\prime}$, in order to determine the eigenvalue $\hat{\omega}$ in terms of $\Delta^{\prime}$. In this limit, when $\hat{\omega} \rightarrow 0, \quad E_{\|} \propto(\varphi-\psi) \rightarrow 0$, $p \rightarrow \frac{p_{0}^{\prime}}{\hat{\omega} T_{e} n_{0}^{\prime}} \psi$ and we can ignore the momentum flux, the solutions of eqn. (147) should behave as $\quad \psi \sim s^{v}, v=-\frac{1}{2} \pm \sqrt{-D_{I}}$ in the limit $s \rightarrow \infty$. However, as they stand, they do not lead to $(\varphi-\psi) \rightarrow 0$. In fact, it is necessary to consider an intermediate region consisting of two sub-layers: (i) a transition layer around $s=s_{1} \sim f_{t}^{1 / 2}\left(m_{i} / m_{e}\right)^{1 / 4}$, where an ion contribution to the electrical conductivity enters; and another (ii), around somewhat larger values of $s$, $s=s_{1} \sim\left(\chi_{\|, e} / \chi_{\|, i}\right)^{1 / 2} \sim\left(m_{i} / m_{e}\right)^{1 / 4}$, where parallel ion thermal transport forces $t_{i} \rightarrow\left(T_{i} T_{e}\right)\left(\eta_{i} \psi / \hat{\omega}\right)$ and ensures $(\varphi-\psi) \rightarrow 0$. Clearly $s_{1}$ and $s_{2}$ are not very different and we can treat them together.

Let us first consider the simplification of the governing equations when $s>>1$.

The electron continuity equation (143) becomes: 


$$
\begin{aligned}
& n-\frac{\varphi}{\hat{\omega}}+\left\langle\frac{\mu_{0} p_{0 e}}{B^{2}}\right\rangle p\left(1-\frac{1+\eta_{e}}{\hat{\omega}}\right)-\kappa C\left(p_{e}-(\varphi-\psi)-\frac{1+\eta_{e}}{\hat{\omega}} \psi\right) \\
& +s^{2}\left[\left(1-1.31 f_{t}\right)\left(p_{e}-(\varphi-\psi)-\frac{1+\eta_{e}}{\hat{\omega}} \psi\right)+0.34\left(1-0.69 f_{t}\right)\left(t_{e}-\frac{\eta_{e}}{\hat{\omega}} \psi\right)\right] \\
& \left.\left.-\frac{\kappa s}{\hat{\omega}} \frac{\mathrm{d}}{\mathrm{d} s}\left[\left(\left\langle\frac{I^{2}}{R^{2} B^{2}}\right\rangle-\frac{1}{\left\langle B^{2}\right\rangle}\left\langle\frac{I^{2}}{R^{2}}\right\rangle\right) p+\frac{f_{t}}{\left\langle B^{2}\right\rangle}\left\langle\frac{I^{2}}{R^{2}}\right\rangle\left(1.67 p-1.19 t_{e}-3.12 t_{i}\right)-\left\langle\frac{I^{2}}{R^{2} B^{2}}\right\rangle\left(p_{i}+\varphi\right)+\frac{1.17}{\left\langle B^{2}\right\rangle}\left(1-f_{t}\right)\right\rangle \frac{I^{2}}{R^{2}}\right\rangle t_{i}\right] \\
& +\frac{\kappa}{\hat{\omega}}\left(\left\langle\frac{I^{2}}{R^{2} B^{2}}\right\rangle-\frac{1}{\left\langle B^{2}\right\rangle}\left\langle\frac{I^{2}}{R^{2}}\right\rangle\right) \frac{\mathrm{d}}{\mathrm{d} s}\left[s\left(p_{e}-(\varphi-\psi)-\frac{1+\eta_{e}}{\hat{\omega}} \psi\right)\right] \\
& +1.67 f_{t} \frac{\kappa}{\hat{\omega}} \frac{1}{\left\langle B^{2}\right\rangle}\left\langle\frac{I^{2}}{R^{2}}\right\rangle \frac{\mathrm{d}}{\mathrm{d} s}\left[s\left(p_{e}-(\varphi-\psi)-\frac{1+\eta_{e}}{\hat{\omega}} \psi\right)\right]=0 .
\end{aligned}
$$

However, this last equation is dominated by its second term, which requires:

$$
\left(1-1.31 f_{t}\right)\left(p_{e}-(\varphi-\psi)-\frac{1+\eta_{e}}{\hat{\omega}} \psi\right)+0.34\left(1-0.69 f_{t}\right)\left(t_{e}-\frac{\eta_{e}}{\hat{\omega}} \psi\right) \cong 0
$$

A similar balance, but with different coefficients, appears in the electron thermal equation (144), so we can conclude that:

$$
\left(p_{e}-(\varphi-\psi)-\frac{1+\eta_{e}}{\hat{\omega}} \psi\right) \cong 0, \quad\left(t_{e}-\frac{\eta_{e}}{\hat{\omega}} \psi\right) \cong 0 \Rightarrow n \cong \varphi-\psi+\frac{\psi}{\hat{\omega}}
$$

so that eqn. (153) simplifies further:

$$
\begin{gathered}
n-\frac{\varphi}{\hat{\omega}}+\left\langle\frac{\mu_{0} p_{0 e}}{B^{2}}\right\rangle p\left(1-\frac{1+\eta_{e}}{\hat{\omega}}\right)+s^{2}\left[\left(1-1.31 f_{t}\right)\left(p_{e}-(\varphi-\psi)-\frac{1+\eta_{e}}{\hat{\omega}} \psi\right)+0.34\left(1-0.69 f_{t}\right)\left(t_{e}-\frac{\eta_{e}}{\hat{\omega}} \psi\right)\right] \\
-\frac{\kappa}{\hat{\omega}} s \frac{\mathrm{d}}{\mathrm{d} s}\left[\left(\left\langle\frac{I^{2}}{R^{2} B^{2}}\right\rangle-\frac{1}{\left\langle B^{2}\right\rangle}\left\langle\frac{I^{2}}{R^{2}}\right\rangle\right) p+\frac{f_{t}}{\left\langle B^{2}\right\rangle}\left\langle\frac{I^{2}}{R^{2}}\right\rangle\left(1.67 p-1.19 t_{e}-3.12 t_{i}\right)-\left\langle\frac{I^{2}}{R^{2} B^{2}}\right\rangle\left(p_{i}+\varphi\right)+\frac{1.17}{\left\langle B^{2}\right\rangle}\left\langle\frac{I^{2}}{R^{2}}\right\rangle t_{i}\right] \\
\quad 0 .
\end{gathered}
$$

With the results (154), the vorticity equation (147) reduces to 


$$
\begin{array}{r}
s \frac{\mathrm{d}^{2}}{\mathrm{~d} s^{2}}(s \psi)+\hat{\omega}\left(D_{I}+\frac{1}{4}\right)\left[\frac{\psi}{\hat{\omega}}+\frac{\left(1+\frac{T_{e}}{T_{i}}\right)(\varphi-\psi)+\left(t_{i}-\frac{T_{i}}{T_{e}} \frac{\eta_{i} \psi}{\hat{\omega}}\right)}{\left(1+T_{e} / T_{i}+\eta_{e}+\eta_{i} T_{i} / T_{e}\right)}\right], \\
-\frac{\mu_{0} p_{0 e}}{\kappa} \frac{\left\langle 1 /|\nabla \chi|^{2}\right\rangle}{\left\langle R^{-2}\right\rangle} \hat{\omega} s \frac{\mathrm{d}}{\mathrm{d} s}\left(1+\frac{T_{e}}{T_{i}}\right)(\varphi-\psi)+\left(t_{i}-\frac{T_{i}}{T_{e}} \frac{\eta_{i} \psi}{\hat{\omega}}\right)=0
\end{array}
$$

where we have neglected the viscous term, which is valid for $s>\left(m_{i} / m_{e}\right)^{3 / 8}\left(\hat{\delta}_{e} / v_{* e}\right)^{1 / 2}$, with $\hat{\delta}_{e}$ the semi-collisional width normalised to the plasma minor radius and $v_{*_{e}}$ the electron collisionality parameter. Ampère's law (146) retains its form.

However, the simplified vorticity equation (157) does not reduce to the ideal form and we must consider the intermediate layer: $s \sim s_{1}, s_{2}$. To address this. we first calculate the correction to the ion parallel velocity arising from the parallel ion pressure gradient - this is achieved by modifying eqn. (21) to give an equation for the correction to the ion distribution function $\hat{h}_{1 i}$

$$
\left\langle\frac{B}{v_{\|}} C_{i i}\left(\hat{h}_{1 i}\right)\right\rangle=\frac{\mathrm{i} \ell I q^{\prime} x}{q}\left\langle\frac{1}{R^{2}}\right\rangle h_{0 i} .
$$

Using the model ion-ion collision operator (27), we can calculate the resulting modification to the ion velocity arising from $\hat{h}_{1 i}$, obtaining 


$$
\hat{U}_{\| i}=-\frac{f_{c}}{f_{t}} \frac{B}{\left\langle B^{2}\right\rangle} \frac{\mathrm{i} \ell I q^{\prime} x}{q}\left\langle\frac{1}{R^{2}}\right\rangle \frac{T_{i}}{m_{i}\left\{v_{i i}\right\}}\left(\left(1+\frac{T_{e}}{T_{i}}\right)(\varphi-\psi)-0.17\left(t_{i}-\frac{T_{i}}{T_{e}} \frac{\eta_{i}}{\hat{\omega}} \psi\right)\right),
$$

where we have used the results in eqn. (155), which are valid at large $s$.

We see that in the absence of the small ion-electron collision frequency, the friction with trapped ions determines the bulk ion velocity. This velocity changes the parallel electron velocity term proportional to $s^{2}$ appearing in the electron continuity equation (143). This additional contribution modifies the behaviour (155) at large $s$ :

$$
\begin{array}{r}
\left(1-1.31 f_{t}\right)\left(p_{e}-(\varphi-\psi)-\frac{1+\eta_{e}}{\hat{\omega}} \psi\right)+034\left(1-0.69 f_{t}\right)\left(t_{e}-\frac{\eta_{e}}{\hat{\omega}} \psi\right) \\
-\delta_{i}\left[\left(1+\frac{T_{e}}{T_{i}}\right)(\varphi-\psi)-0.17\left(t_{i}-\frac{T_{i}}{T_{e}} \frac{\eta_{i}}{\hat{\omega}} \psi\right)\right]=0,
\end{array}
$$

where, using results for the collision integrals from Appendix A,

$$
\delta_{i}=0.95 \frac{\left(1-f_{t}\right)}{f_{t}}\left(\frac{T_{i}}{T_{e}}\right)^{5 / 2}\left(\frac{m_{e}}{m_{i}}\right)^{1 / 2}<<1 .
$$

In this limit, eqn. (146) reduces to

$$
\frac{\mathrm{d}^{2}}{\mathrm{~d} s^{2}}(s \psi)=-\delta_{i}\left(\frac{\hat{\omega}}{\kappa}\right)^{2} \frac{\mu_{0} p_{0 e}}{I^{2}\left\langle R^{-2}\right\rangle^{2}}\left\langle\frac{B^{2}}{|\nabla \chi|^{2}}\right\rangle s\left[\left(1+\frac{T_{e}}{T_{i}}\right)(\varphi-\psi)-0.17\left(t_{i}-\frac{T_{i}}{T_{e}} \frac{\eta_{i} \psi}{\hat{\omega}}\right)\right],
$$

We can also calculate the corresponding parallel ion heat flux, finding it is dominated by the convective component due to the inverse dependence on the trapped particle fraction of the parallel ion flow in eqn. (159): 


$$
\begin{aligned}
& \left\langle\frac{\mathrm{i} \ell q^{\prime} x}{R^{2} B q} Q_{\| i}\right\rangle= \\
& \quad \frac{5}{2} \frac{f_{c}^{2}}{f_{t}}\left(\frac{\ell I q^{\prime} x}{q}\right)^{2} \frac{1}{\left\langle B^{2}\right\rangle}\left\langle\frac{1}{R^{2}}\right\rangle^{2} \frac{T_{i}}{m_{i}\left\{v_{i i}\right.}\left(\left(1+\frac{T_{e}}{T_{i}}\right)(\varphi-\psi)+\left(t_{i}-\frac{T_{i}}{T_{e}} \frac{\eta_{i}}{\hat{\omega}} \psi\right)\left(\frac{\left\{v_{i i} u^{2}\right\}}{\left\{v_{i i}\right\}}-\frac{3}{2}\right)\right) n_{0} T_{\mathrm{i}}
\end{aligned}
$$

This modifies eqn. (145):

$$
\begin{aligned}
& \frac{2.63}{D}\left(\frac{\kappa}{\hat{\omega}}\right)^{2} \frac{m_{i} \tau_{e i}}{m_{e} \tau_{i}} \frac{1}{\left\langle B^{2}\right\rangle}\left\langle\frac{I^{2}}{R^{2}}\right\rangle \frac{\mathrm{d}^{2}}{\mathrm{~d} s^{2}}\left[\left(\left\langle\frac{|\nabla \chi|^{2}}{B^{2}}\right\rangle\left\langle\frac{1}{R^{2}}\right\rangle+\left\langle\frac{I^{2}}{R^{2}}\right\rangle\left(\left\langle\frac{1}{B^{2}}\right\rangle-\frac{1}{\left\langle B^{2}\right\rangle}\right)\right)+\frac{0.46 f_{t}}{\left\langle B^{2}\right\rangle}\left\langle\frac{I^{2}}{R^{2}}\right\rangle\right] t_{i} \\
& +t_{i}-\frac{T_{i}}{T_{e}} \frac{\eta_{i}}{\hat{\omega}} \psi=-\frac{5}{3 D}\left\langle\frac{\mu_{0} p_{0 e}}{B^{2}}\right\rangle\left(1+\frac{T_{i}}{T_{e}} \frac{\left(1+2 \eta_{i}\right)}{\hat{\omega}}\left(1+\frac{T_{i}}{T_{e}}+\eta_{e}+\eta_{i} \frac{T_{i}}{T_{e}}\right)\right) \frac{\psi}{\hat{\omega}} \\
& -\frac{T_{i}}{D T_{e}}\left(1-\frac{\left(1+\eta_{i}\right)}{\hat{\omega}}+\frac{5}{3}\left(1+\frac{T_{i}}{T_{e}}\right)\left(\frac{\kappa}{\hat{\omega}} C+\left\langle\frac{\mu_{0} p_{0 e}}{B^{2}}\right\rangle\left(\frac{T_{e}}{T_{i}}+\frac{\left(1+2 \eta_{i}\right)}{\hat{\omega}}\right)\right)-1.59 \delta_{i} s^{2}\right)(\varphi-\psi),
\end{aligned}
$$

where

$$
D=1+\frac{5}{3}\left\langle\frac{\mu_{0} p_{0 e}}{B^{2}}\right\rangle\left[1+\frac{T_{i}}{T_{e}} \frac{\left(1+2 \eta_{i}\right)}{\hat{\omega}}+2 \frac{T_{i}}{T_{e}} \frac{\kappa}{\hat{\omega}} C\right]+0.27 \delta_{i} s^{2}
$$

and we have again used evaluations of collision integrals given in Appendix A.

The new terms proportional to $\delta_{i}$ dominate eqns. (162) and (164) when $s>s_{1}, s_{2}$ and require

$$
\varphi-\psi \rightarrow 0, \quad t_{i}-\frac{T_{i}}{T_{e}} \frac{\eta_{i} \psi}{\hat{\omega}} \rightarrow 0
$$


When all of conditions (155) and (166) are satisfied we see that eqn. (157) does indeed reduce to the marginal ideal MHD equation:

$$
s \frac{\mathrm{d}^{2}}{\mathrm{~d} s^{2}}(s \psi)+\left(D_{I}+\frac{1}{4}\right) \psi=0
$$

To discuss the transition though the intermediate region we combine eqns. (157) and (146), modifying the latter to take account of eqn. (160).

Using the expressions (155) for $n$ and $t_{e}$ to express $p$ and $p_{i}$ in terms of $\varphi, \psi$ and $t_{i}$, we obtain

$$
\begin{aligned}
& \frac{1.17}{\left\langle B^{2}\right\rangle} \frac{\hat{\omega}}{\kappa}\left\langle\frac{B^{2}}{|\nabla \chi|^{2}}\right\rangle \frac{\mu_{0} p_{0 e}}{\left\langle 1 / R^{2}\right\rangle}\left(1-f_{t}\right) s \frac{\mathrm{d} t_{i}}{\mathrm{~d} s} \\
& -\frac{\mu_{0} p_{0 e}}{\left\langle 1 / R^{2}\right\rangle} \frac{\hat{\omega}}{\kappa}\left[\frac{1}{\hat{\omega}}\left(1+\eta_{e}+\frac{T_{i}}{T_{e}}\left(1+\eta_{i}\right)\right)\left\langle\frac{1}{|\nabla \chi|^{2}}\right\rangle+\left[1-\frac{1+\eta_{e}}{\hat{\omega}}\right] \frac{\left\langle B^{2}|\nabla \chi|^{2}\right\rangle\left\langle 1 / R^{2} B^{2}\right\rangle}{\left\langle 1 / R^{2}\right\rangle}\right] s \frac{\mathrm{d} \psi}{\mathrm{d} s} \\
& -\left[\frac{\left(D_{I}+1 / 4\right) \hat{\omega}}{\left(1+\eta_{e}+T_{i} / T_{e}\left(1+\eta_{i}\right)\right)}+\left\langle\frac{\mu_{0} p_{0 e}}{B^{2}}\right\rangle \frac{\mu_{0} p_{0 e}\left\langle B^{2} /\left.\nabla \chi\right|^{2}\right\rangle}{I^{2}\left\langle 1 / R^{2}\right\rangle^{2}}\left(1-\frac{1+\eta_{e}}{\hat{\omega}}\right) \frac{\hat{\omega}}{\kappa^{2}}+0.17 \frac{\mu_{0} p_{0 e}\left\langle B^{2} /\left.\nabla \chi\right|^{2}\right\rangle}{I^{2}\left\langle 1 / R^{2}\right\rangle^{2}}\left(\frac{\hat{\omega}}{\kappa}\right)^{2} \delta_{i} s^{2}\right] t_{i} \\
& -\left[\frac{\left(D_{I}+1 / 4\right)}{\left(1+\eta_{e}+T_{i} / T_{e}\left(1+\eta_{i}\right)\right)}+\left\langle\frac{\mu_{0} p_{0 e}}{B^{2}}\right\rangle \frac{\mu_{0} p_{0 e}\left\langle B^{2} /\left.\nabla \chi\right|^{2}\right\rangle}{I^{2}\left\langle 1 / R^{2}\right\rangle^{2}}\left(1-\frac{1+\eta_{e}}{\hat{\omega}}\right) \frac{\hat{\sigma}}{\kappa^{2}}\right]\left(1+\eta_{e}+\frac{T_{i}}{T_{e}}\right) \psi \\
& +0.17 \frac{\hat{\omega}}{\kappa^{2}} \frac{\mu_{0} p_{0 e}\left\langle B^{2} /\left.\nabla \chi\right|^{2}\right\rangle}{I^{2}\left\langle 1 / R^{2}\right\rangle^{2}} \eta_{i} \frac{T_{i}}{T_{e}} \delta_{i} s^{2} \psi-\frac{\left(D_{I}+1 / 4\right)\left(1+T_{i} / T_{e}\right) \hat{\omega}}{\left(1+\eta_{e}+T_{i} / T_{e}\left(1+\eta_{i}\right)\right)}(\varphi-\psi) \\
& -\left[1-\frac{1}{\hat{\omega}}+\left\langle\frac{\mu_{0} p_{0 e}}{B^{2}}\right\rangle \frac{\mu_{0} p_{0 e}\left\langle B^{2} /\left.\nabla \chi\right|^{2}\right\rangle}{I^{2}\left\langle 1 / R^{2}\right\rangle^{2}}\left(1-\frac{1+\eta_{e}}{\hat{\omega}}\right)\left(\frac{\hat{\omega}}{\kappa}\right)^{2}-\frac{\mu_{0} p_{0 e}\left\langle B^{2} /\left.\nabla \chi\right|^{2}\right\rangle}{I^{2}\left\langle 1 / R^{2}\right\rangle^{2}}\left(\frac{\hat{\omega}}{\kappa}\right)^{2}\left(1+\frac{T_{e}}{T_{i}}\right) \delta_{i} s^{2}\right](\varphi-\psi)=0 .
\end{aligned}
$$

Then eqns. (157) and (168), with $t_{i}$ given by eqn. (164), provide a fourth order system of equations for $\varphi$ and $\psi$ to connect the semi-collisional electron 
layer solutions to the ideal MHD region through the region $s \sim s_{1}, s_{2}$. Their boundary conditions at large $s$ are to match to the correct ratio of large and small solutions arising from the ideal region and ensure $\varphi-\psi \rightarrow 0$. Thus the jump in the ratio of large to small solutions through this layer, and hence the layer $\Delta^{\prime}$, can be computed.

\section{Conclusions}

We have derived a set of equations to describe the linear stability of semicollisional, neoclassical toroidal plasma in general geometry, albeit provided that: (i) the fraction of trapped particles is small to justify the use of a model, pitch angle scattering, collision operator; and (ii) that the ions are magnetised. The assumption that the ions are magnetised may require low magnetic shear or $T_{i}<<T_{e}$, otherwise a non-local model for the ion response will be needed (Fitzpatrick 1989).

This set of equations comprises a pair of second order radial differential equations for the electron density, $\tilde{n}$ (or pressure, $\tilde{p}_{e}$ ) and temperature, $\tilde{T}_{e}$ perturbations (eqns. (116) and (117)) and one for the ion temperature perturbation, $\tilde{T}_{i}$, eqn. (118), in terms of the perturbed electrostatic potential, $\Phi$, and parallel vector potential, $\tilde{A}_{\|}$, described by the potential $\Psi$, with these two 
quantities in turn being determined by Ampère's law, eqn. (122), with eqn. (123) for the parallel current, and the vorticity equation, eqn. (131). The perturbed parallel magnetic field is given simply by eqn. (58).

The analysis of neoclassical electron physics utilised the Spitzer functions (Spitzer \& Harm 1953), where we remark that parallel gradients in perturbed electron temperature that have no counterpart in standard neoclassical theory (Helander \& Sigmar 2002), necessitate the introduction of the second Spitzer function corresponding to parallel electron thermal conduction. The vorticity equation requires a calculation of radial collisional transport of toroidal angular momentum and we have needed to generalise the treatment by Wong and Chan (Wong \& Chan 2005 to arbitrary geometry, as in Appendix B, although still assuming a small number of trapped particles. This calculation employed the model ion collision operator. Analytical evaluations of the coefficients in the large aspect ratio limit are presented there; these are consistent with the results of Wong and Chan (Wong \& Chan 2005).

The introduction of general toroidal equilibria and the use of the Spitzer functions extend the treatment of electron neoclassical physics given by Fitzpatrick (Fitzpatrick 1989), as well as providing a more consistent treatment of the neoclassical radial transport than in the work of Connor et al. (Connor et al. 2009). The general geometry aspect also means we have needed to include classical collisional transport. 
The resultant equations, summarised in normalised form in eqns. (143) - (148), are equivalent to a twelfth order system of radial differential equations if all effects are retained - it reduces to tenth order if we neglect the radial angular momentum transport. However, the relatively large ion thermal diffusivity means one can treat the ion temperature as a constant over the semi-collisional layer. (This also greatly simplifies the expression for the radial transport of toroidal angular momentum given in eqn. (136).) The system of equations then reduces to tenth order and one only needs to solve a simplified version of the ion thermal equation in the intermediate region, $s \sim\left(m_{i} / m_{e}\right)^{1 / 4}$, where the system reduces further to a fourth order set. A simpler version of eqns. (143) - (146) in which we ignore the subdominant classical and Pfirsch-Schlüter fluxes relative to the banana contributions, the smaller azimuthal fluxes and effects proportional to $\beta_{e}=2 \mu_{0} p_{0 e} / B^{2}<<1$, is presented in eqns. (149) - (152). The vorticity equation (147) is unaffected by these approximations.

The solution of these equations in the narrow radial layer around a low-order resonant surface needs to be matched to 'external' solutions of the marginal ideal MHD equations. However, to achieve this matching, as pointed out by Fitzpatrick (Fitzpatrick 1989), we need to consider the intermediate layer where small corrections arising from the ion contribution to the electrical conductivity and the parallel ion thermal diffusivity enter and ensure that the perturbed parallel electric field vanishes. Since this correction is determined by the 
friction of the passing ion population with the trapped ones, rather than the schematic ion sound model suggested by Fitzpatrick (Fitzpatrick 1989), we were able to provide an explicit form for it. This intermediate region is described by a fourth order set of equations, eqns. (157) and (164) together with eqn. (168), that allow a proper matching to the ideal MHD region. Continuing the set of equations through this layer allows us to obtain a dispersion relation

$$
\hat{\Delta}(\omega+\mathrm{i} \gamma)=\Delta^{\prime}
$$

where $\Delta^{\prime}$ is the toroidal tearing mode stability parameter (Glasser et al. 1975) and $\hat{\Delta}(\omega+\mathrm{i} \gamma)$ is obtained from the solution of the layer equations, allowing for the effect of the intermediate layer.

Although our analysis is linear, the treatment of the semi-collisional, neoclassical electron physics could be generalised to describe the evolution of non-linear neoclassical tearing mode islands, extending the analysis given by Wilson et al. (Wilson et al. 1996) for magnetised ions to this more collisional regime, or be used in conjunction with a numerical treatment for ions when the width of the poloidal ion Larmor orbit is comparable to the island width (Imada et al. 2016). Alternatively, it could serve to incorporate neoclassical physics in the collisional model of Smolyakov (Smolyakov 1993). 


\section{REFERENCES}

Cohen, R. S., Spitzer, L., Jr. \& McR. Routly, P. 1950 The electrical conductivity of an ionized gas. Phys. Review 80 (2), 230-238.

Connor, J. W., Grimm, R. C., Hastie, R. J. \& Keeping, P. 1973 The conductivity of a toroidal plasma. Nucl. Fusion 13 (2), 211-14.

Connor, J. W., Hastie, R. J. \& Helander, P. 2009 Linear tearing mode stability equations for a low collisionality toroidal plasma. Plasma Phys. Control. Fusion 51 (1), 015009.1-26.

Fitzpatrick, R. 1989 Linear stability of low mode number tearing modes in the banana collisionality regime. Phys. Fluids B 1 (12), 2381-96.

Glasser, A. H., Greene, J. M. \& Johnson, J. L. 1975 Resistive instabilities in general toroidal plasma configurations. Phys. Fluids 18 (7), 875-88.

Hahm, T. S. 1988 Neoclassical tearing modes in a tokamak. Phys. Fluids 31 (12), 3709-12.

Helander, P. \& Sigmar, D. J. 2002 Collisional Transport in Magnetized Plasmas. Cambridge University Press, Cambridge, UK.

Imada, K., Connor, J. W. \& Wilson, H. R. 2016 Finite banana width effect on NTM threshold physics, $43^{\text {rd }}$ EPS Conference on Plasma Physics, Leuven, July 4-8, 2016, paper 05.128. 
Mercier, C. 1960 Un critere necessaire de stabilite hydromagnetique pour un plasma en symetrie de revolution Nucl. Fusion 1(1), 47-53.

Rosenbluth, M. N., Hazeltine, R. D. \& Hinton, F. L. 1972 Plasma transport in toroidal confinement systems. Phys. Fluids 15 (1), 116-140.

Rutherford, P. H., Kovrizhnykh L. M., Rosenbluth, M. N. \& Hinton, F. L. 1970 Effect of longitudinal electric field on toroidal diffusion. Phys. Rev. Lett. 25 (16), 1090-93.

Smolyakov, A. 1993 Nonlinear evolution of tearing modes in inhomogeneous plasmas. Plasma Phys. Control. Fusion 35 (6), 657-87.

Spitzer, L., Jr, \& Harm, R. 1953 Transport Phenomena in a completely ionized gas. Phys. Review 89 (1), 977 - 981.

Tang, W. M., Connor, J. W. \& Hastie, R. J. 1980 Kinetic-ballooning-mode theory in general geometry. Nucl. Fusion 20 (11), 1439-53.

Wong, S. K. \& Chan, V. S. 2005 The neoclassical angular momentum flux in the large aspect ratio limit. Phys. Plasmas 12 (9), 092513.1 - 13.

Wilson, H. R., Connor, J. W., Hastie, R. J. \& Hegna, C. C. 1996 Threshold for neoclassical magnetic island in a low collision frequency tokamak. Phys. Plasmas 3 (1), 248-265. 


\section{Appendix A: Some Collisional Integrals}

Here we list numerical evaluations of the various integrals, including those involving the Spitzer functions $D^{(1)}$ and $D^{(2)}$ tabulated in Spitzer and Harm (Spitzer \& Harm 1953) (to be precise, $D^{(1)}=-D / A$ and $D^{(2)}=2 D / B$ as given in Tables I and II of Spitzer \& Harm 1953):

$$
\begin{gathered}
\left\{v_{e}\right\}=1.153 v_{0 e},\left\{u^{2} v_{e}\right\}=1.284 v_{0 e},\left\{u^{4} v_{e}\right\}=2.701 v_{0 e}, \\
\left\{\frac{D^{(1)}(u)}{u}\right\}=-2.616,\left\{\frac{D^{(2)}(u)}{u}\right\}=-0.898,\left\{u \mathrm{D}^{(1)}(u)\right\}=-8.297,\left\{u D^{(2)}(u)\right\}=-4.782, \\
\left\{v_{e} \frac{D^{(1)}(u)}{u}\right\}=-1.666 v_{0 e},\left\{v_{e} \frac{D^{(2)}(u)}{u}\right\}=-0.040 v_{0 e}, \\
\left\{v_{e} u D^{(1)}(u)\right\}=-2.972 v_{0 e},\left\{v_{e} u D^{(2)}(u)\right\}=-0.738 v_{0 e}, \\
\left\{v_{e}\left(\frac{D^{(1)}(u)}{u}\right)^{2}\right\}=3.432 v_{0 e},\left\{v_{e} \frac{D^{(1)}(u) D^{(2)}(u)}{u^{2}}\right\}=0.605 v_{0 e},\left\{v_{e}\left(\frac{D^{(2)}(u)}{u}\right)^{2}\right\}=0.566 v_{0 e}, \\
\left\{v_{i i}\right\}=0.401 v_{0 i},\left\{u^{2} v_{i i}\right\}=0.532 v_{0 i},\left\{u^{4} v_{i i}\right\}=20.62 / 19 v_{0 i},\left\{u^{4} / v_{i i}\right\}=100.37 / v_{0 i} .
\end{gathered}
$$




\section{Appendix B: Radial Toroidal Angular Momentum Transport}

There are two contributions from the radial transport of momentum appearing in the vorticity equation (131): the classical one, $\left\langle\Pi^{C}\right\rangle$, and the neoclassical one, $\left\langle\Pi^{N C}\right\rangle$. We evaluate these in this Appendix:

\section{(a) Classical radial angular momentum transport}

The expression for $\mathrm{d}^{2}\left\langle\Pi^{C}\right\rangle / \mathrm{d} x^{2}$ involves $\left\langle\sum_{j} e \int \mathrm{d}^{3} v L_{j}^{2} C_{j}\left(g_{0 j} L_{j}^{2}\right)\right\rangle$, where the sum is dominated by the ions. Thus, substituting for $g_{0 i}$ from eqns. (8) and (22) and recalling the definition of $L_{i}$ below eqn. (2), we obtain

$$
\left\langle\Pi^{C}\right\rangle=e\left(\frac{m_{i}}{e}\right)^{4}\left\langle\frac{|\nabla \chi|^{4}}{B^{4}}\right) \frac{\mathrm{d}^{2}}{\mathrm{~d} x^{2}}\left\{\int \mathrm{d}^{3} \boldsymbol{v} v_{r}^{2} C_{i}\left[v_{r}^{2}\left(\frac{\tilde{p}_{i}}{p_{0 i}}+\frac{e \Phi}{T_{i}}+\left(\frac{m_{i} v^{2}}{2 T_{i}}-\frac{5}{2}\right) \frac{\tilde{T}_{i}}{T_{i}}\right) f_{0 i}\right]\right\} .
$$

The collision integrals in eqn. (B.1) can be evaluated using the entropy functional $S\left(\hat{f}\left(\boldsymbol{v}^{\prime}\right), \hat{g}(\boldsymbol{v})\right) \quad$ (Helander $\quad \& \quad$ Sigmar $\left.\quad 2002\right), \quad$ with $\hat{f}=v_{\|}^{\prime 3}$ and $\hat{g}=v_{\|}\left(m_{i} v^{2} / 2 T_{i}-5 / 2\right)$. The quantity $S$ can be easily calculated using Cartesian co-ordinates in velocity space, labelling the direction parallel to the magnetic field as the $x$-direction, and introducing $\boldsymbol{u}=\left(\boldsymbol{v}^{\prime}-\boldsymbol{v}\right) / 2$ and $\boldsymbol{w}=\left(\boldsymbol{v}^{\prime}+\boldsymbol{v}\right) / 2$ to execute the velocity space integrations. The result is 


$$
\left\langle\Pi^{C}\right\rangle=-\frac{n_{0} e}{5 \tau_{i}}\left(\frac{2 m_{i} T_{i}}{e^{2}}\right)^{2}\left\langle\frac{|\nabla \chi|^{4}}{B^{4}}\right\rangle \frac{\mathrm{d}^{2}}{\mathrm{~d} x^{2}}\left\{\frac{\tilde{p}_{i}}{p_{0 i}}+\frac{e \Phi}{T_{i}}+3 \frac{\tilde{T}_{i}}{T_{i}}\right\} .
$$

\section{(b) Neoclassical radial angular momentum transport}

Equation (128) requires the evaluation of

$$
\left\langle\Pi^{N C}\right\rangle=\left\langle\sum_{j} \int \mathrm{d}^{3} \boldsymbol{v} m_{j} \frac{I}{B} v_{\|} v_{d r j} h_{2 j}\right\rangle,
$$

representing the radial transport of the toroidal angular momentum arising from the parallel flow: $R v_{\varphi}=I v_{\|} / B$. Noting that it is dominated by the ion contribution, we express this quantity as

$$
\left\langle\Pi^{N C}\right\rangle=I\left\langle\int \mathrm{d}^{3} v \frac{I v_{\|}}{R^{2} B q} \frac{\partial}{\partial \theta}\left(\frac{I m_{i}^{2} v_{\|}^{2}}{2 e B^{2}}\right) h_{2 i}\right\rangle .
$$

In order to avoid the need to calculate $h_{2 i}$, we follow Wong and Chan (Wong and Chan 2005) in defining the adjoint function, $g$ :

$$
\frac{I v_{\|}}{R^{2} B q} \frac{\partial g}{\partial \theta}+C_{i i}(g)=-\frac{I v_{\|}}{R^{2} B q} \frac{\partial}{\partial \theta}\left(\frac{I m_{i}^{2} v_{\|}^{2}}{2 e B^{2}}\right) f_{0 i},
$$

so that, using the self-adjointness of the collision operator, integrations by parts and the gyro-kinetic equation for $h_{2 i}$, we obtain

$$
\left\langle\Pi^{N C}\right\rangle=\frac{I m_{i}^{3}}{e^{2}}\left\langle\int \mathrm{d}^{3} \boldsymbol{v} \frac{g}{f_{0 i}}\left(\frac{I v_{\|}}{R^{2} B q} \frac{\partial}{\partial \theta}\left(\frac{I v_{\|}}{B}\right) h_{2 i}^{\prime}\right)\right\rangle .
$$


We use the gyro-kinetic equation for $h_{2 i}$ and eqn. (B.5) for $g$, which we expand for weak collisions, so that we can integrate it to give

$$
g=-\frac{\operatorname{Im}_{i}^{2} v_{\|}^{2}}{2 e B^{2}} f_{0 i}+G(\lambda, v)
$$

where $G$ is given by the collisional constraint that follows from eqn. (B.5):

$$
\left\langle\frac{B}{v_{\|}} C_{i i}\left(-\frac{I m_{i}^{2} v_{\|}^{2}}{2 e B^{2}} f_{0 i}+G(\lambda, v)\right)\right\rangle=0 .
$$

Thus

$$
g=\frac{I m_{i}^{2} v^{2}}{2 e}\left(\frac{\lambda}{B}-\int_{0}^{\lambda} \mathrm{d} \lambda \frac{\left\langle v_{\|} / B\right\rangle}{\left\langle v_{\|}\right\rangle}\right) f_{0 i}=\frac{I m_{i}^{2} v^{2}}{2 e}\left(\lambda\left(\frac{1}{B}-\left\langle\frac{1}{B}\right\rangle\right)-\int_{0}^{\lambda} \mathrm{d} \lambda \frac{\left\langle v_{\|} / B-v_{\|}\langle 1 / B\rangle\right\rangle}{\left\langle v_{\|}\right\rangle}\right) f_{0 i},
$$

where the final integral is small in the trapped particle fraction.

Performing further integrations by parts on eqn. (B.6), we finally obtain

$$
\left\langle\Pi^{N C}\right\rangle=\left\langle\Pi^{N C}\right\rangle^{A}+\left\langle\Pi^{N C}\right\rangle^{B},
$$

where

$$
\left\langle\Pi^{N C}\right\rangle^{A}=\frac{I m_{i}}{e}\left\langle\int \mathrm{d}^{3} \boldsymbol{v} \frac{I v_{\|}}{B}\left(-\frac{I}{6 e}\left(\frac{m_{i} v_{\|}}{B}\right)^{2}+\frac{G}{f_{0 i}}\right) C_{i i}\left(h_{1 i}^{\prime}\right)\right\rangle
$$

and 


$$
\left\langle\Pi^{N C}\right\rangle^{B}=-\frac{I m_{i}}{e}\left\langle\int \mathrm{d}^{3} \boldsymbol{v} \frac{I v_{\|}}{B} \frac{h_{1 i}^{\prime}}{f_{0 i}} C_{i i}(g)\right\rangle,
$$

where, $h_{1 i}^{\prime}$ is given by eqn. (28).

It is helpful to re-write eqn. (B.11) as

$$
\left\langle\Pi^{N C}\right\rangle^{A}=\frac{I m_{i}}{e}\left\langle\int \mathrm{d}^{3} \boldsymbol{v} \frac{I v_{\|}}{B}\left(\frac{I}{3 e}\left(\frac{m_{i} v_{\|}}{B}\right)^{2}+\frac{g}{f_{0 i}}\right) C_{i i}\left(h_{1 i}^{\prime}\right)\right\rangle .
$$

The first term can be evaluated by using the model collision operator (27) acting on $h_{1 i}$ given in eqn. (64). Since the ion-ion collision operator vanishes when acting on a displaced Maxwellian distribution, only the perturbed temperature gradient terms in expressions (66) and (67) contribute. First we consider the banana contribution (67). Integrating by parts in $\lambda$, using the results (30), (32) and the evaluations in Appendix A, we obtain

$$
\left\langle\Pi^{B a n}\right\rangle^{A 1}=0.37 \frac{n_{0} e}{\left\langle B^{2}\right\rangle \tau_{i}}\left(\frac{I^{2} m_{i} T_{i}}{e^{2}}\right)^{2}\left(G^{(0)}-\left\langle\frac{1}{B^{2}}\right\rangle\right) \frac{\mathrm{d}^{2}}{\mathrm{~d} x^{2}}\left(\frac{\tilde{T}_{i}}{T_{i}}\right),
$$

where

$$
G^{(0)}=\frac{15\left\langle B^{2}\right\rangle^{2}}{4}\left\langle\frac{1}{B^{2}} \int_{0}^{1 / B_{\text {Max }}} \mathrm{d} \lambda \frac{\lambda(1-\lambda B)}{\langle\sqrt{1-\lambda B}\rangle}\right\rangle .
$$

For the Pfirsch-Schlüter contribution we apply the collision operator directly to eqn. (66). The result is 


$$
\left\langle\Pi^{P S}\right\rangle^{A 1}=0.37 \frac{n_{0} e}{\tau_{i}}\left(\frac{I^{2} m_{i} T_{i}}{e^{2}}\right)^{2}\left[\left\langle\frac{1}{B^{4}}\right\rangle-\frac{1}{\left\langle B^{2}\right\rangle}\left\langle\frac{1}{B^{2}}\right\rangle\right] \frac{\mathrm{d}^{2}}{\mathrm{~d} x^{2}}\left(\frac{\tilde{T}_{i}}{T_{i}}\right)
$$

Thus the total contribution to $\left\langle\Pi^{N C}\right\rangle^{A 1}$ is given by the sum of results (B.14) and (B.16):

$$
\left\langle\Pi^{N C}\right\rangle^{A 1}=0.37 \frac{n_{0} e}{\tau_{i}}\left(\frac{I^{2} m_{i} T_{i}}{e^{2}}\right)^{2}\left\{\frac{G^{(0)}}{\left\langle B^{2}\right\rangle^{2}}+\left\langle\frac{1}{B^{4}}\right\rangle-\frac{2}{\left\langle B^{2}\right\rangle}\left\langle\frac{1}{B^{2}}\right\rangle\right\} \frac{\mathrm{d}^{2}}{\mathrm{~d} x^{2}}\left(\frac{\tilde{T}_{i}}{T_{i}}\right)
$$

Equation (B.13) also has a contribution from the function $g$, which is labelled $\left\langle\Pi^{N C}\right\rangle^{A 2}$. The contribution to this from the banana term in $h_{1 i}$, eqn. (67), can be evaluated using eqn. (B.12) for $g$. We employ the model collision operator, recalling the banana contribution to $U_{\| i}^{*}=0$, and integrate by parts in $\lambda$ twice. Since $h_{1 i}^{B a n}$ is localised, we can approximate $\partial\left(\lambda v_{\|}^{2} \partial g / \partial \lambda\right) / \partial \lambda \cong-B v^{2} \lambda \partial g / \partial \lambda$. The integral term in expression (B.12) for $g$ only contributes significantly as $\lambda$ enters the trapping region, but remains small (i.e., $O(\delta \lambda)$ compared to the first term, where $\delta \lambda$ is the trapped width in $\lambda$ ) and can be ignored. Finally, inserting expression (67) for $h_{1 i}^{B a n}$, performing the velocity space integrals recalling the results in Appendix $A$, and changing the order of the $\lambda$-integration and the \langle\rangle averaging operation, we obtain 


$$
\left\langle\Pi^{B a n}\right\rangle^{A 2}=0.37 \frac{n_{0} e}{\tau_{i}\left\langle B^{2}\right\rangle}\left(\frac{I^{2} m_{i} T_{i}}{e^{2}}\right)^{2}\left[4\left(\frac{1}{B^{2}}\right\rangle-\left\langle\frac{1}{B}\right\rangle^{2}-\frac{G^{(1)}}{\left\langle B^{2}\right\rangle}\right] \frac{\mathrm{d}^{2}}{\mathrm{~d} x^{2}}\left(\frac{\tilde{T}_{i}}{T_{i}}\right),
$$

where

$$
\begin{aligned}
\frac{G^{(1)}}{3\left\langle B^{2}\right\rangle}= & \frac{15}{4} \int_{0}^{1 / B_{\text {Min }}} \mathrm{d} \lambda \lambda \frac{\langle B \sqrt{1-\lambda B}\rangle}{\langle\sqrt{1-\lambda B}\rangle}\left\langle\frac{\sqrt{1-\lambda B}}{B}\right\rangle+\frac{15}{16}\left\langle B^{2}\right\rangle\left\langle\frac{1}{B}\right\rangle^{1 / B_{\text {Max }}} \frac{\mathrm{d} \lambda \lambda^{2}}{\langle\sqrt{1-\lambda B}\rangle} . \\
& -\frac{15}{8}\left\langle B^{2}\right\rangle \int_{0}^{1 / B_{\text {Max }}} \mathrm{d} \lambda \lambda \frac{1}{\langle\sqrt{1-\lambda B}\rangle}\left\langle\frac{\sqrt{1-\lambda B}}{B}\right\rangle^{1 / B_{\text {Max }}} \int_{\lambda}^{\frac{\mathrm{d} \lambda^{\prime}}{\left\langle\sqrt{1-\lambda^{\prime} B}\right\rangle}} .
\end{aligned}
$$

The calculation of the Pfirsch-Schlüter contribution from eqn. (66) is more straightforward. We evaluate the collision operator acting on $h_{1 i}^{P S}$, again neglect the small integral tern in $g$ and evaluate the velocity space integrals using the results in Appendix A. The result is:

$$
\left\langle\Pi^{P S}\right\rangle^{A 2}=0.37 \frac{n_{0} e}{\tau_{i}}\left(\frac{I^{2} m_{i} T_{i}}{e^{2}}\right)^{2}\left[\left\langle\frac{1}{B^{4}}\right\rangle-\frac{1}{\left\langle B^{2}\right\rangle}\left\langle\frac{1}{B^{2}}\right\rangle-\left\langle\frac{1}{B}\right\rangle\left(\left\langle\frac{1}{B^{3}}\right\rangle-\frac{1}{\left\langle B^{2}\right\rangle}\left\langle\frac{1}{B}\right\rangle\right)\right] \frac{\mathrm{d}^{2}}{\mathrm{~d} x^{2}}\left(\frac{\tilde{T}_{i}}{T_{i}}\right)
$$

Thus the total contribution to $\left\langle\Pi^{N C}\right\rangle^{A 2}$ from results (B.18) and (B.20) is

$$
\left\langle\Pi^{N C}\right\rangle^{A 2}=0.37 \frac{n_{0} e}{\tau_{i}}\left(\frac{I^{2} m_{i} T_{i}}{e^{2}}\right)^{2}\left[\left\langle\frac{1}{B^{4}}\right\rangle+\frac{3}{\left\langle B^{2}\right\rangle}\left\langle\frac{1}{B^{2}}\right\rangle-\left\langle\frac{1}{B^{3}}\right\rangle\left\langle\frac{1}{B}\right\rangle-\frac{G^{(1)}}{\left\langle B^{2}\right\rangle^{2}}\right] \frac{\mathrm{d}^{2}}{\mathrm{~d} x^{2}}\left(\frac{\tilde{T}_{i}}{T_{i}}\right) .
$$


Combining the results from eqns. (B.17) and (B.21), we finally obtain $\left\langle\Pi^{N C}\right\rangle^{A}=0.37 \frac{n_{0} e}{\tau_{i}}\left(\frac{I^{2} m_{i} T_{i}}{e^{2}}\right)^{2}\left[2\left\langle\frac{1}{B^{4}}\right\rangle-\left\langle\frac{1}{B^{3}}\right\rangle\left\langle\frac{1}{B}\right\rangle+\frac{1}{\left\langle B^{2}\right\rangle}\left\langle\frac{1}{B^{2}}\right\rangle+\frac{\left(G^{(0)}-G^{(1)}\right)}{\left\langle B^{2}\right\rangle^{2}}\right] \frac{\mathrm{d}^{2}}{\mathrm{~d} x^{2}}\left(\frac{\tilde{T}_{i}}{T_{i}}\right)$.

Turning to the quantity $\left\langle\Pi^{N C}\right\rangle^{B}$, we first observe that the periodicity constraint (B.11) allows us to rewrite eqn. (B.9) as

$$
\left\langle\Pi^{N C}\right\rangle^{B}=-\frac{I m_{i}}{e}\left\langle\int \mathrm{d}^{3} \boldsymbol{v} \frac{I v_{\|}}{B f_{0 i}}\left(\frac{h_{1 i}^{\prime} v_{\|}}{B}-\left\langle\frac{h_{1 i}^{\prime} v_{\|}}{B}\right\rangle\right) C_{i i}(g)\right\rangle .
$$

We introduce the three contributions to $h_{1 i}$ from eqn. (64), use the model ionion collision operator (27), substitute

$$
\frac{\partial g}{\partial \lambda}=\frac{I m_{i}^{2} v^{2}}{2 e}\left(\frac{1}{B}-\frac{\left\langle v_{\|} / B\right\rangle}{\left\langle v_{\|}\right\rangle}\right) f_{0 i}
$$

as follows from eqn. (B.12), and integrate by parts in $\lambda$. The contribution from $h_{1}^{\text {Flow }}$ is:

$$
\left\langle\Pi^{F l o w}\right\rangle^{B}=0.80 \frac{n_{0} e}{\tau_{i}} \frac{1}{\left\langle B^{2}\right\rangle}\left(\frac{I^{2} m_{i} T_{i}}{e^{2}}\right)^{2}\left[\left\langle\frac{1}{B^{2}}\right\rangle-\frac{G^{(2)}}{\left\langle B^{2}\right\rangle}\right] \frac{\mathrm{d}^{2}}{\mathrm{~d} x^{2}}\left(\frac{\tilde{p}_{i}}{p_{0 i}}+\frac{e \Phi}{T_{i}}-1.17 \frac{\tilde{T}_{i}}{T_{i}}\right),
$$

where 


$$
G^{(2)}=\frac{15\left\langle B^{2}\right\rangle^{2}}{4}\left\langle\frac{1}{B^{2}} \int_{0}^{1 / B_{\text {Max }}} \lambda \mathrm{d} \lambda \frac{\langle B \sqrt{1-\lambda B}\rangle}{\langle\sqrt{1-\lambda B}\rangle}\left\langle\frac{\sqrt{1-\lambda B}}{B}\right\rangle\right\rangle \quad,
$$

that from $h_{1}^{P S}$ is:

$$
\left\langle\Pi^{P S}\right\rangle^{B}=0.80 \frac{n_{0} e}{\tau_{i}}\left(\frac{I^{2} m_{i} T_{i}}{e^{2}}\right)^{2}\left(\left\langle\frac{1}{B^{4}}\right\rangle-\frac{1}{\left\langle B^{2}\right\rangle}\left\langle\frac{1}{B^{2}}\right\rangle+\frac{G^{(3)}}{\left\langle B^{2}\right\rangle^{2}}\right) \frac{\mathrm{d}^{2}}{\mathrm{~d} x^{2}}\left(\frac{\tilde{p}_{i}}{p_{0 i}}+\frac{e \Phi}{T_{i}}-0.25 \frac{\tilde{T}_{i}}{T_{i}}\right)
$$

where

$$
G^{(3)}=\frac{15\left\langle B^{2}\right\rangle^{2}}{4} \int_{0}^{1 / B_{\text {Min }}} \mathrm{d} \lambda \lambda \frac{1}{\langle\sqrt{1-\lambda B}\rangle}\left\langle\frac{\sqrt{1-\lambda B}}{B}\right\rangle\left(\frac{\langle B \sqrt{1-\lambda B}\rangle}{\left\langle B^{2}\right\rangle}-\left\langle\frac{\sqrt{1-\lambda B}}{B}\right\rangle\right)
$$

and from $h_{1}^{B a n}$ is:

$$
\left\langle\Pi^{\text {Ban }}\right\rangle^{B}=0.74 \frac{n_{0} e}{\tau_{i}\left\langle B^{2}\right\rangle}\left(\frac{I^{2} m_{i} T_{i}}{e^{2}}\right)^{2}\left[\left\langle\frac{1}{B^{2}}\right\rangle-\frac{1}{\left\langle B^{2}\right\rangle}\left(\frac{G^{(1)}}{3}+G^{(4)}\right)\right] \frac{\mathrm{d}^{2}}{\mathrm{~d} x^{2}}\left(\frac{\tilde{T}_{i}}{T_{i}}\right),
$$

where

$$
G^{(4)}=\frac{15\left\langle B^{2}\right\rangle^{2}}{4} \int_{0}^{1 / B_{M a x}} \mathrm{~d} \lambda \lambda \frac{1}{\langle\sqrt{1-\lambda B}\rangle}\left(\frac{1}{\langle\sqrt{1-\lambda B}\rangle}\left\langle\frac{\sqrt{1-\lambda B}}{B}\right\rangle\left\langle\frac{1-\lambda B}{B}\right\rangle-\left\langle\frac{1-\lambda B}{B^{2}}\right\rangle\right) .
$$


Consequently, combining results (B.25), (B.27) and (B.29), we obtain the result

$$
\begin{aligned}
& \left\langle\Pi^{N C}\right\rangle^{B}=0.80 \frac{n_{0} e}{\tau_{i}}\left(\frac{I^{2} m_{i} T_{i}}{e^{2}}\right)^{2}\left[\left\langle\frac{1}{B^{4}}\right\rangle+\frac{\left(G^{(3)}-G^{(2)}\right)}{\left\langle B^{2}\right\rangle^{2}}\right] \frac{\mathrm{d}^{2}}{\mathrm{~d} x^{2}}\left(\frac{\tilde{p}_{i}}{p_{0 i}}+\frac{e \Phi}{T_{i}}\right) \\
& -\frac{n_{0} e}{\tau_{i}}\left(\frac{I^{2} m_{i} T_{i}}{e^{2}}\right)^{2}\left[0.2\left\langle\frac{1}{B^{4}}\right\rangle+\frac{\left(0.74\left(G^{(1)} / 3+G^{(4)}\right)-0.94 G^{(2)}+0.2 G^{(3)}\right)}{\left\langle B^{2}\right\rangle^{2}}\right] \frac{\mathrm{d}^{2}}{\mathrm{~d} x^{2}}\left(\frac{\tilde{T}_{i}}{T_{i}}\right)
\end{aligned}
$$

Finally, combining results (B.22) and (B.31), we obtain the expression for the neoclassical toroidal angular viscosity:

$$
\begin{aligned}
& \left\langle\Pi^{N C}\right\rangle=0.80 \frac{n_{0} e}{\tau_{i}}\left(\frac{I^{2} m_{i} T_{i}}{e^{2}}\right)^{2}\left[\left\langle\frac{1}{B^{4}}\right\rangle+\frac{\left(G^{(3)}-G^{(2)}\right)}{\left\langle B^{2}\right\rangle^{2}}\right] \frac{\mathrm{d}^{2}}{\mathrm{~d} x^{2}}\left(\frac{\tilde{p}_{i}}{p_{0 i}}+\frac{e \Phi}{T_{i}}\right) \\
& +\frac{n_{0} e}{\tau_{i}\left\langle B^{2}\right\rangle}\left(\frac{I^{2} m_{i} T_{i}}{e^{2}}\right)^{2}\left[0.37\left(\frac{1}{\left\langle B^{2}\right\rangle}\left\langle\frac{1}{B^{2}}\right\rangle-\left\langle\frac{1}{B}\right\rangle\left\langle\frac{1}{B^{3}}\right\rangle\right)+0.54\left\langle\frac{1}{B^{4}}\right\rangle\right] \frac{\mathrm{d}^{2}}{\mathrm{~d} x^{2}}\left(\frac{\tilde{T}_{i}}{T_{i}}\right) \\
& +\frac{n_{0} e}{\tau_{i}\left\langle B^{2}\right\rangle^{2}}\left(\frac{I^{2} m_{i} T_{i}}{e^{2}}\right)^{2}\left(0.37 G^{(0)}-0.62 G^{(1)}+0.94 G^{(2)}-0.2 G^{(3)}-0.74 G^{(4)}\right) \frac{\mathrm{d}^{2}}{\mathrm{~d} x^{2}}\left(\frac{\tilde{T}_{i}}{T_{i}}\right) .
\end{aligned}
$$

Result (B.32) is the expression appearing in eqn. (136).

It is interesting to consider the large aspect ratio limit with $B=B_{0}(1-\varepsilon \cos \theta), \varepsilon=r / R_{0}<<1$. Analytical evaluation of the coefficients in eqn. (B.32) in this limit yields

$$
\begin{aligned}
& \left\langle\Pi^{N C}\right\rangle \\
& =\frac{n_{0} e}{\tau_{i}}\left(\frac{I^{2} m_{i} T_{i}}{e^{2} B_{0}^{2}}\right)^{2}\left[0.8 \varepsilon^{2} \frac{\mathrm{d}^{2}}{\mathrm{~d} x^{2}}\left(\frac{\tilde{p}_{i}}{p_{0 i}}+\frac{e \Phi}{T_{i}}\right)-0.74 \varepsilon^{3 / 2} \frac{\mathrm{d}^{2}}{\mathrm{~d} x^{2}}\left(\frac{\tilde{T}_{i}}{T_{i}}\right)+O\left(\varepsilon^{2}\right) \frac{\mathrm{d}^{2}}{\mathrm{~d} x^{2}}\left(\frac{\tilde{T}_{i}}{T_{i}}\right)\right],
\end{aligned}
$$


in agreement with Wong and Chan (Wong \& Chan 2005). 